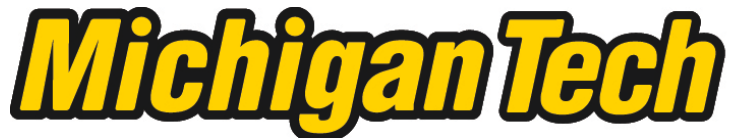 \\ Michigan Technological University Create the Future Digital Commons @ Michigan Tech
}

2011

\section{Automation of shear-wave splitting parameter determination of local earthquakes at Yellowstone : application as indicator of crustal stress and temporal variation}

Nicole D. McMahon

Michigan Technological University

Follow this and additional works at: https://digitalcommons.mtu.edu/etds

Part of the Geophysics and Seismology Commons

Copyright 2011 Nicole D. McMahon

Recommended Citation

McMahon, Nicole D., "Automation of shear-wave splitting parameter determination of local earthquakes at Yellowstone : application as indicator of crustal stress and temporal variation ", Master's Thesis, Michigan Technological University, 2011.

https://doi.org/10.37099/mtu.dc.etds/333

Follow this and additional works at: https://digitalcommons.mtu.edu/etds

Part of the Geophysics and Seismology Commons 


\title{
AUTOMATION OF SHEAR-WAVE SPLITTING PARAMETER DETERMINATION OF LOCAL EARTHQUAKES AT YELLOWSTONE: APPLICATION AS INDICATOR OF CRUSTAL STRESS AND TEMPORAL VARIATION
}

\section{By}

Nicole D. McMahon

\begin{abstract}
A THESIS
Submitted in partial fulfillment of the requirements for the degree of MASTER OF SCIENCE

(Geophysics)
\end{abstract}

MICHIGAN TECHNOLOGICAL UNIVERSITY

2011

(C) 2011 Nicole D. McMahon 
This thesis, "Automation of shear-wave splitting parameter determination of local earthquakes at Yellowstone: Application as indicator of crustal stress and temporal variation," is hereby approved in partial fulfillment of the requirements for the Degree of MASTER OF SCIENCE IN GEOPHYSICS.

Department of Geological and Mining Engineering and Sciences

Signatures:

Thesis Advisor

Dr. Gregory P. Waite

Committee Member

Dr. Simon A. Carn

Committee Member

Dr. Petra Huentemeyer

Department Chair

Dr. Wayne D. Pennington

Date 


\section{Table of Contents}

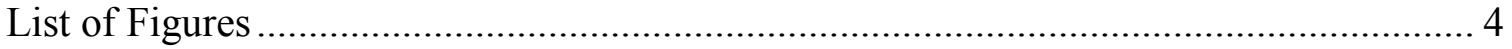

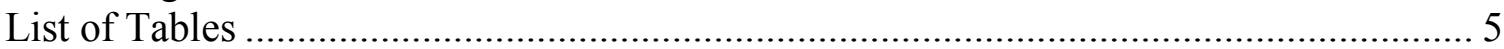

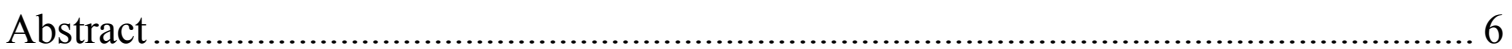

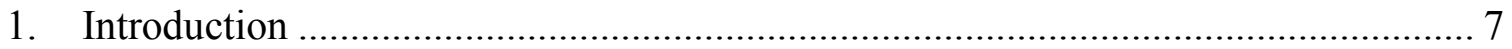

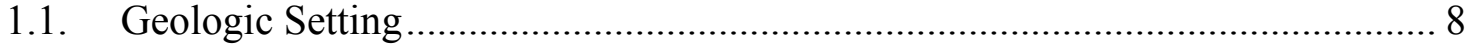

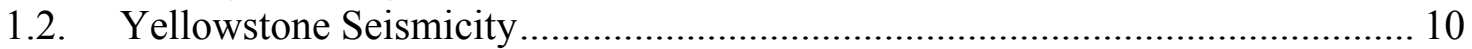

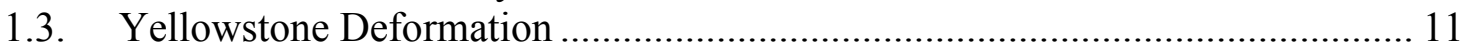

1.4. Shear-wave Splitting .............................................................................. 13

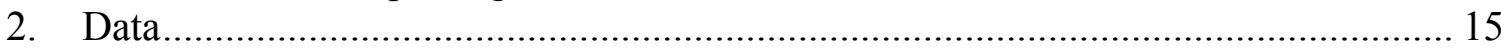

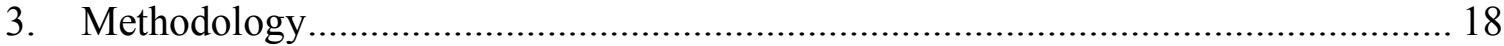

3.1. Shear-wave Splitting Parameter Calculations ................................................ 18

3.2. The Analysis Window .............................................................................. 19

3.3. Cluster Analysis ................................................................................... 20

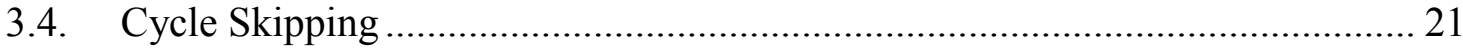

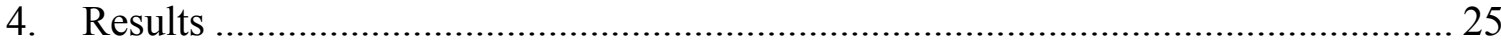

4.1. The Silver and Chan (1991) Method................................................................ 25

4.2. The Levin et al. (1999) Method .................................................................... 32

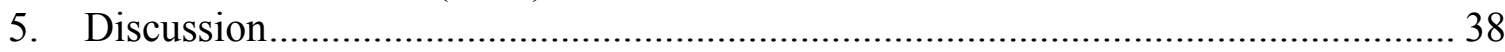

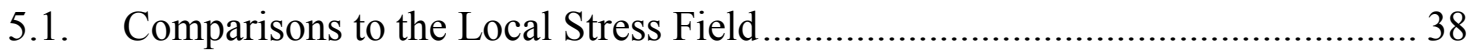

5.2. Temporal Variation in Fast Directions..................................................... 41

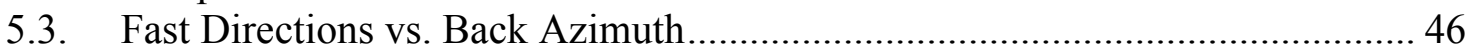

5.4. Fast Directions vs. Frequency Band........................................................... 47

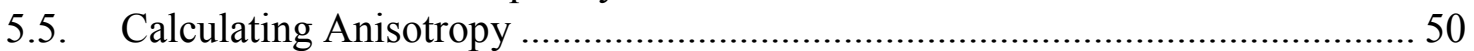

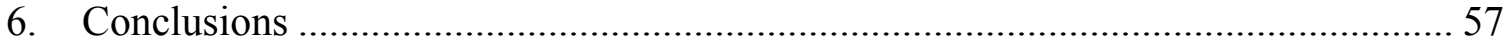

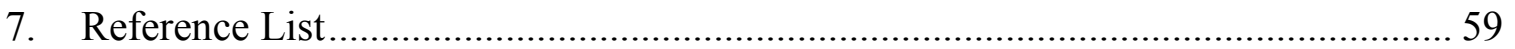

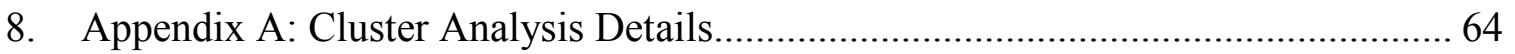

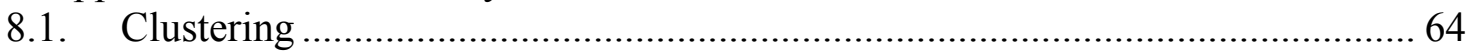

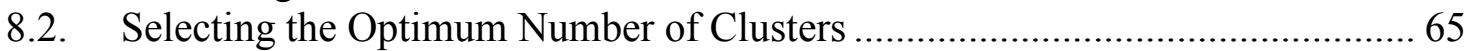

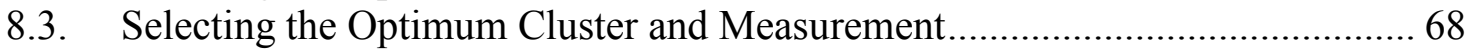

9. Appendix B: Figure 1.2 Copyright License Agreement ........................................ 70 


\section{List of Figures}

Figure 1.1 Map of the Yellowstone and Eastern Snake River Plain

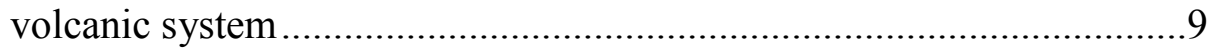

Figure 1.2 Deformation details of the Yellowstone volcanic field ............................. 12

Figure 1.3 Diagrams of shear-wave splitting terminology .......................................14

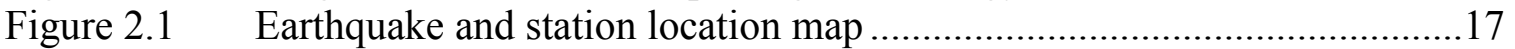

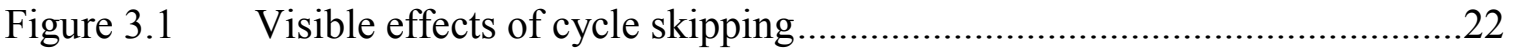

Figure 4.1 Silver and Chan (1991) shear-wave splits map.....................................26

Figure 4.2 Rose plots of Silver and Chan (1991) fast polarization directions ............27

Figure 4.3 Event diagnostic example 1 ...........................................................28

Figure 4.4 Event diagnostic example 1 - corrected for cycle skipping .....................29

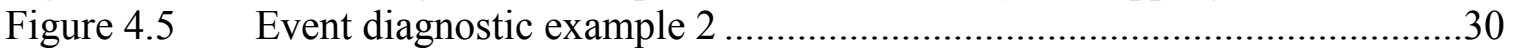

Figure 4.6 Event diagnostic example 2 - corrected for cycle skipping ......................31

Figure 4.7 SC shear-wave splits map - corrected for cycle skipping ........................34

Figure 4.8 Rose plots of Silver and Chan (1991) fast polarization directions corrected for cycle skipping..........................................................35

Figure 4.9 Rose plots of Levin et al. (1999) fast directions .....................................36

Figure 4.10 Rose plots of Levin et al. (1999) fast directions - corrected for

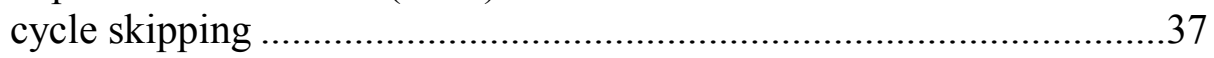

Figure 5.1 Map of preferred fast directions, local stress and strain .........................39

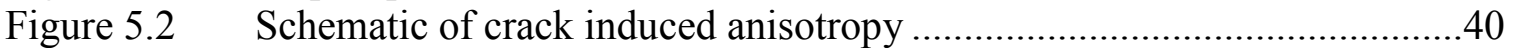

Figure 5.3 Fast direction as a function of time....................................................43

Figure 5.4 Fast direction vs. 60-day and 180-day average on station YHH ...............44

Figure 5.5 Fast direction by year at station YHH..............................................45

Figure 5.6 Fast direction as a function of back azimuth .........................................48

Figure 5.7 Fast direction vs. $10^{\circ}$ back azimuth average on stations YHH and YMR.

Figure 5.8 Fast direction vs. frequency band: B208, H17A, LKWY .........................51

Figure 5.9 Fast direction vs. frequency band: YFT, YHH, YJC ...............................52

Figure 5.10 Fast direction vs. frequency band: YMP, YMR, YNR ............................53 


\section{List of Tables}

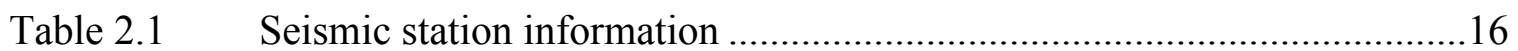

Table 3.1 Time window analysis and grid search parameters ...................................21

Table 3.2 Cluster analysis and cycle skipping parameters......................................23

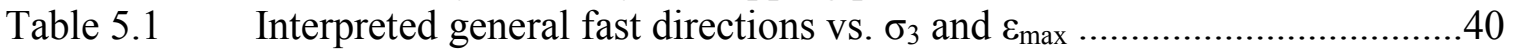

Table 5.2 Calculated percent anisotropies and crack densities ..................................56

Table $8.1 \quad$ Cluster analysis parameters....................................................................69 


\section{Abstract}

Shear-wave splitting can be a useful technique for determining crustal stress fields in volcanic settings and temporal variations associated with activity. Splitting parameters were determined for a subset of local earthquakes recorded from 2000-2010 at Yellowstone. Analysis was automated using an unsupervised cluster analysis technique to determine optimum splitting parameters from 270 analysis windows for each event. Six stations clearly exhibit preferential fast polarization values sub-orthogonal to the direction of minimum horizontal compression. Yellowstone deformation results in a local crustal stress field differing from the regional field dominated by NE-SW extension, and fast directions reflect this difference rotating around the caldera maintaining perpendicularity to the rim. One station exhibits temporal variations concordant with identified periods of caldera subsidence and uplift. From splitting measurements, we calculated a crustal anisotropy of $\sim 17-23 \%$ and crack density $\sim 0.12-0.17$ possibly resulting from stress-aligned fluid filled microcracks in the upper crust and an active hydrothermal system. 


\section{Acknowledgements}

I would like to thank my advisor Greg Waite for the tremendous amount of assistance and encouragement offered in the course of my research. Thanks to Simon Carn and Petra Huentemeyer for sitting on my committee, offering non-seismologists' points of view, and editorial suggestions. The earthquake catalog was obtained from the University of Utah, Salt Lake City, UT, and the MATLAB codes for determining shearwave splitting parameters were written by Derek Schutt and Greg Waite. 


\section{Introduction}

Shear-wave splitting is frequently used to measure anisotropy in the Earth and describe its structure. Shear-wave splitting parameters can be used to describe the medium through which seismic waves have passed. Several techniques for determining these splitting parameters have been developed (e.g., Silver and Chan 1991; Levin et al. 1999; Long and van der Hilst 2005). We apply a semi-automated shear wave measurement technique to local earthquakes in the Yellowstone region in order to determine the direction of crustal stresses as well as define temporal variations related to the deformation of the caldera. By automating determination of shear-wave splitting parameters we dramatically increase the practicality of incorporating large amounts of earthquake data into our study and hopefully improve the quality of results.

\subsection{Geologic Setting}

The Yellowstone volcanic system is one of the largest and most active silicic volcanic systems in the world. The Yellowstone Plateau is the youngest in a 16 Ma series of progressively older volcanic centers defined by a hotspot track extending $800-\mathrm{km}$ southwest along the eastern Snake River Plain to the Oregon-Nevada border (Figure 1.1) (Christiansen 2001; Waite et al. 2005). More than 140 giant silicic eruptions have been identified associated with the hotspot's eastward track (Perkins and Nash 2002). Three cataclysmic eruptions at 2.05, 1.3, and $0.64 \mathrm{Ma}$ formed the presently active 40-km by 70-km caldera and Yellowstone Plateau volcanic field (Christiansen 2001). The most recent eruption at $70 \mathrm{ka}$ is one of more than 50 rhyolite flows occurring since the caldera formation (Christiansen 2001; Christiansen et al. 2007).

Geodetic data reveal that Yellowstone deformation is dominated by the lithospheric extension of the Basin and Range province and the superposition of caldera subsidence and uplift (Smith et al. 2009). Gripp and Gordon (2002) observed the North American Plate at Yellowstone to be moving at an azimuth of $241^{\circ}$, and Smith et al. (2009) 


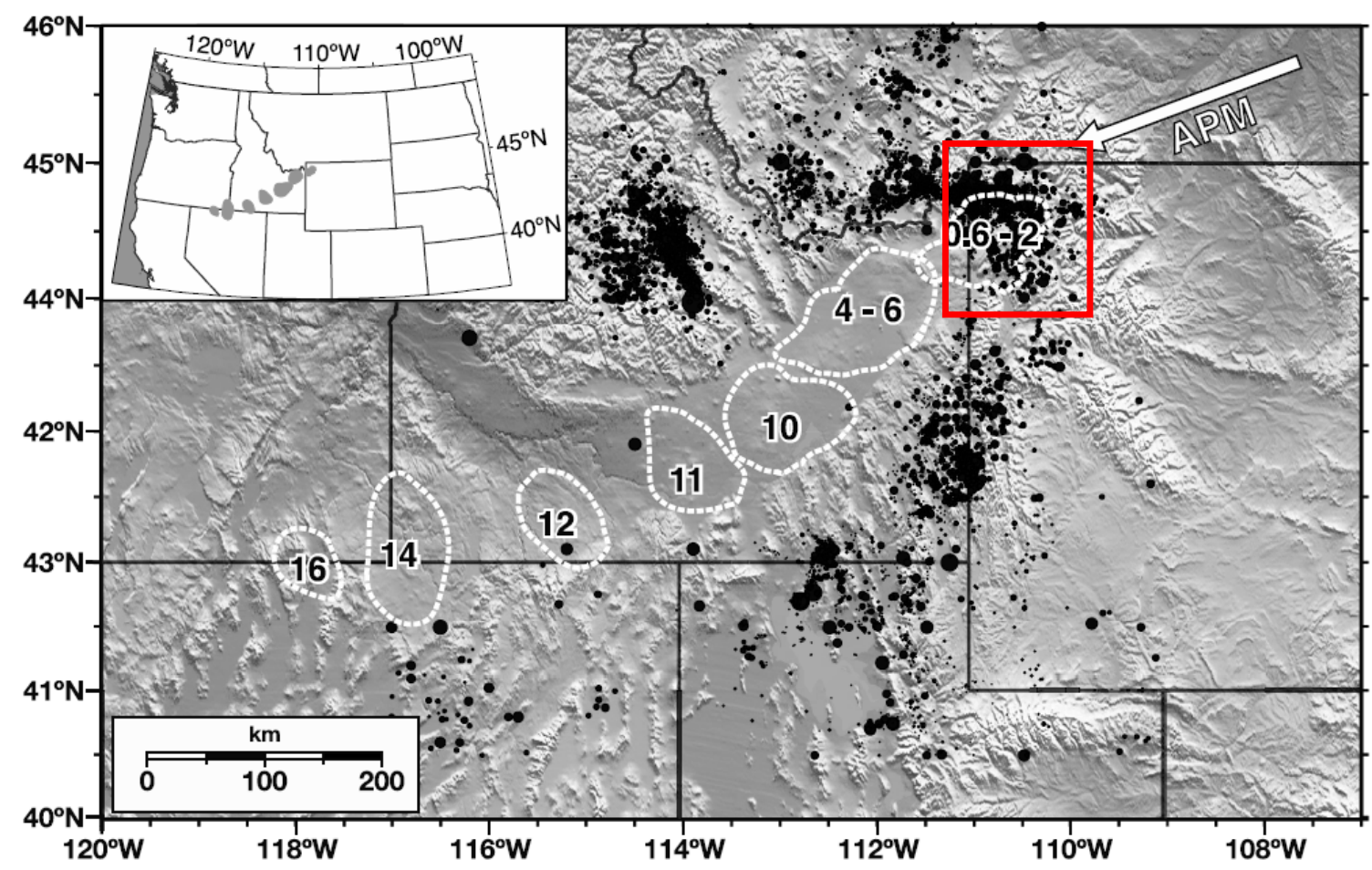

Figure 1.1 Map of the Yellowstone and Eastern Snake River Plain volcanic system. State boundaries are solid black lines. Earthquake epicenters are plotted as black circles. Eruptive centers are white dashed ellipsoids with age in Ma. Apparent plate notion (APM) of $241^{\circ}$ from Gripp and Gordon (2002) is indicated by the white arrow. The area of study is indicated by the red box. There is a topographic and seismic activity high in a parabolic pattern around the Easter Snake River Plain with its apex at Yellowstone.

Waite GP, Smith RB, Allen RM. 2006. $V_{P}$ and $V_{S}$ structure of the Yellowstone hot spot from teleseismic tomography: Evidence for an upper mantle plume. Journal of Geophysical Research, 111( B04303), 2006. Copyright 2006 American Geophysical Union. Reproduced/modified by permission of American Geophysical Union.

determined a southwest extension at the rate of 2-3 mm/yr across the Yellowstone Plateau. This rate of extension is approximately one-fourth of the total Basin and Range opening-rate (Smith et al. 2009).

Crystallizing magma at shallow depths is responsible for the unusually high heat flow in Yellowstone estimated at $\sim 2000 \mathrm{~mW} / \mathrm{m}^{2}$ (combined conductive and convective heat), 3040 times the average continental heat flow (Blackwell 1969; Fournier 1989). 


\subsection{Yellowstone Seismicity}

Yellowstone is the most seismically active area of the 1,300-km long Intermountain Seismic Belt (ISB) extending from Montana to Arizona (Smith and Arabasz 1991). The seismicity in Yellowstone is the result of the interactions between the extending lithosphere of the Basin and Range Province and the Yellowstone volcanic system which can perturb stresses up to 50-km from the hotspot track (Husen and Smith 2004; White et al. 2009). The earthquake activity in Yellowstone is characterized by a low level of background seismicity ( $<100$ earthquakes/per week) interrupted by infrequent spatially and temporally constrained swarms of small shallow earthquakes (Farrell et al. 2009; Farrell et al. 2010). More than 80 swarms have been identified between since 1995 (Farrell et al. 2009).

The largest recorded earthquake swarm in Yellowstone occurred on the northwest rim of the caldera in late 1985 and spanned more than three months with more than 3,000 events of $\mathrm{M}<5$ (Waite and Smith 2002). This swarm coincided with the onset of caldera subsidence after more than 60 years of uplift (Meertens and Smith 1991; Puskas et al. 2007). The largest earthquake recorded in Yellowstone and the surrounding region was the August 1959 Ms7.5 Hebgen Lake, Montana earthquake which occurred 25-km northwest of the caldera (Doser 1985). The largest earthquake recorded in the caldera was the $\mathrm{M}_{\mathrm{L}} 6.0$ event that occurred on June 30, 1975 approximately 8-km from the Norris Junction seismic station (Murdock 1978). The majority of seismic activity in Yellowstone occurs NNW of the caldera between the epicenters of these two large earthquakes (Christiansen 2001; Farrell et al. 2009). Earthquake depths within the caldera are constrained to the upper $5-\mathrm{km}$ due to the shallow depth of the brittle-ductile transition associated with a low velocity zone of crystallizing magma. Earthquakes northwest of the caldera extend to 18-km depth (Smith et al. 2009).

A regional seismic network was first installed in Yellowstone in 1972 by the United States Geological Survey (USGS) (Pitt 1987). Operations were taken over by the University of Utah Seismograph Station in 1991, and the first three-component stations 
were installed in 1995 (Husen and Smith 2004). Since 1995, updates to the regional network as well as additional networks and temporary deployments have significantly added to the amount of three-component data available for analysis.

\subsection{Yellowstone Deformation}

Global Positioning System (GPS) and Interferometric Synthetic Aperture Radar (InSar) measurements have yielded evidence of several caldera uplift and subsidence episodes in the past $\sim 25$ years (Fig. 1.2). From 1987 to 1995, the caldera subsided at a maximum rate of $-14 \pm 3-\mathrm{mm} / \mathrm{yr}$ for a total of $112-\mathrm{mm}$, and from 1995 to 2000 , the caldera uplifted at a rate of $15 \pm 4-\mathrm{mm} / \mathrm{yr}$ for a total of $75 \mathrm{~mm}$. From 2000 to 2003, the northwest portion of the caldera continued uplift, while the central axis subsided (Puskas et al. 2007). In late 2004, Yellowstone began an unprecedented period of accelerated uplift at a rate of 7$\mathrm{cm} / \mathrm{yr}$ and $5-\mathrm{cm} / \mathrm{yr}$ in the northern and the southwest caldera, respectively, three times greater than any previously observed uplift rates (Chang et al. 2007; Chang et al. 2010). In 2006-2008 there was a decrease in the rate of inflation of the caldera and further decrease in 2009 (Chang et al. 2010). Chang et al, (2010) suggest that magmatic intrusions at 7-10-km beneath the caldera are responsible for the uplift since 2004, and a decreasing rate of replenishment from beneath the northeast caldera and an increase of seismic moment release are responsible for the continued, but declining uplift. In January 2010, there was a large earthquake swarm northwest of the caldera near the site of the 1985 swarm, after which the caldera returned to subsidence (Chang et al. 2010).

The Norris Geyser Basin to the northwest of the caldera was characterized by inflation from 1987-2004 at which point the basin began to subside while the main caldera experienced uplift. Chang et al. (Chang et al. 2010) use a deflating source model at 7-13 $\mathrm{km}$ beneath this region to explain the subsidence. Wicks et al. (2006) propose an exchange of basaltic magma between the caldera and the Norris Geyser basin as a model for inversely correlating periods of subsidence and uplift in the two regions. 

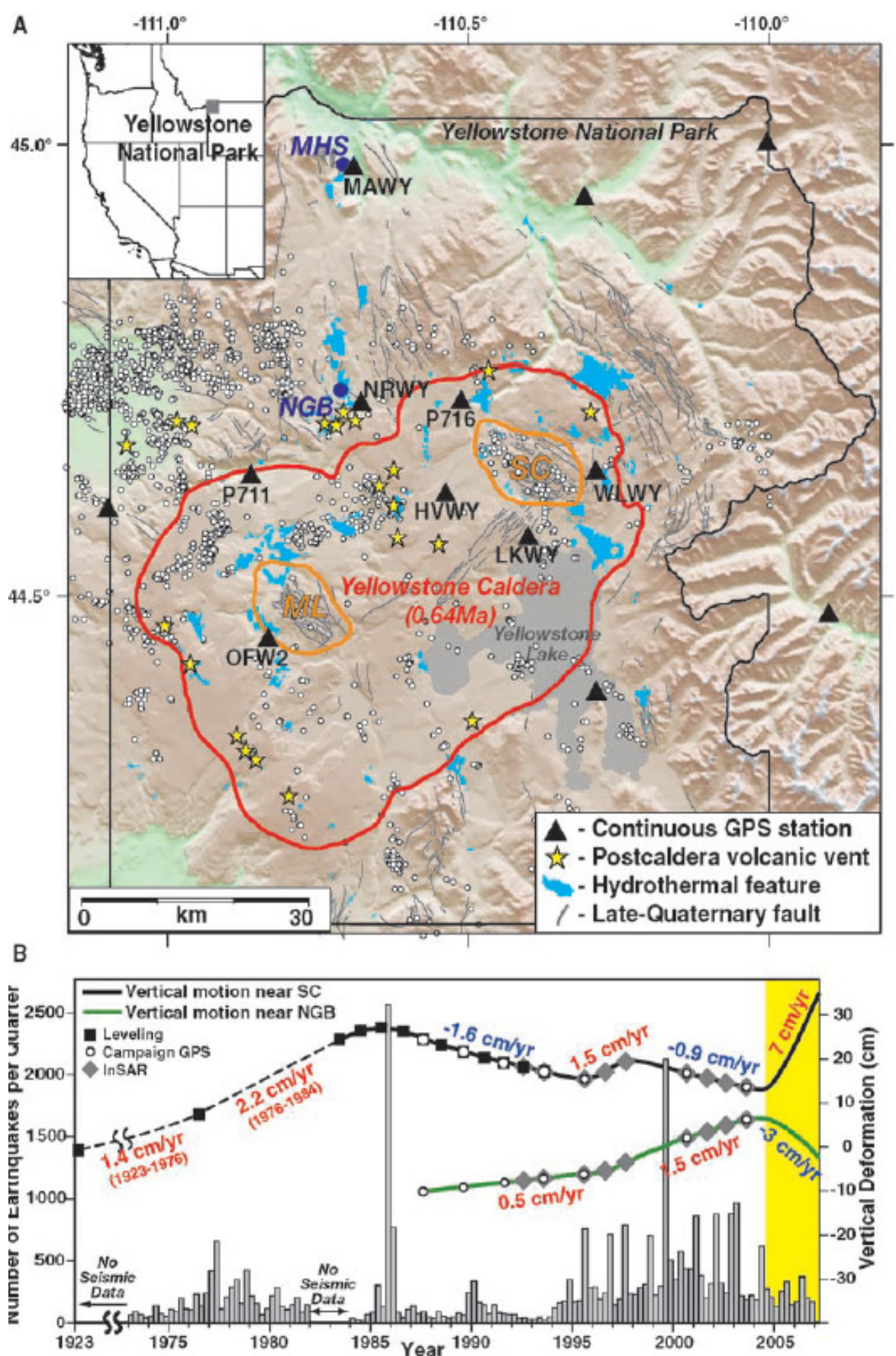

Figure 1.2 Deformation details of the Yellowstone volcanic field. (A) Earthquake epicenters from October 2004-March 2007 are marked by white dots. SC = Sour Creek resurgent dome; $\mathrm{ML}=$ Mallard Lake resurgent dome; NGB = Norris Geyser Basin; MHS = Mammoth Hot Springs. Four character codes are GPS stations. (B) Yellowstone vertical ground motions and quarterly earthquake counts. The yellow shaded area indicates the period of accelerated uplift beginning in 2004.

From Chang WL, Smith RB, Wicks C, Farrell JM, Puskas CM. 2007. Accelerated uplift and magmatic intrusion o the Yellowstone caldera, 2004-2006. Science 318, 952-956. Reprinted with permission from AAAS. See Appendix B for copyright license agreement. 


\subsection{Shear-wave Splitting}

Shear-wave splitting, also known as seismic birefringence, is a phenomenon caused by shear waves passing through an anisotropic medium leading to directional dependence in seismic velocities. When a shear wave propagates through an anisotropic medium, the energy is partitioned into two orthogonally polarized fast and slow waves (Crampin 1981). Split shear waves are described by the parameters $\varphi$, the polarization direction of the fast traveling wave, and $\delta t$, the delay in arrival time between the fast and slow waves (Fig. 1.3). These parameters can reveal details about the medium through which the waves have passed.

Seismic anisotropy describes the directional dependence of wave velocity in an anisotropic medium and is caused by preferential alignment of features such as fractures (e.g., alignment of faults or microcracks in the crust) or anisotropic minerals (e.g., alignment of platy minerals in the crust and olivine crystals in the mantle). An anisotropic medium is one which contains such preferential alignment of features. A three-component seismic station is needed to detect and measure anisotropy because the information on the two horizontal components are used to resolve the differences in the arrivals of fast and slow waves.

Shear-wave splitting has been exploited in both local and teleseismic earthquakes for studies into mantle deformation (e.g., Silver and Chan 1991), earthquake and volcanic eruption forecasting (e.g., Gerst and Savage 2004; Tai et al. 2008), tomography (e.g., Silver and Long 2011), reservoir characterization (e.g., Lou and Rial 1997), and many more. In this study, we utilize shear-wave splitting in local earthquakes as an indicator of crustal stress at Yellowstone and attempt to determine temporal variations related to the deformation of the caldera. Yellowstone is an active volcanic system and determination of the crustal stress field can aid in characterizing the volcanic activity and possible eruption forecasting. Shear-wave splitting provides an opportunity to examine the anisotropy of the upper crust and temporal variations related to changes in the volcanic system. 

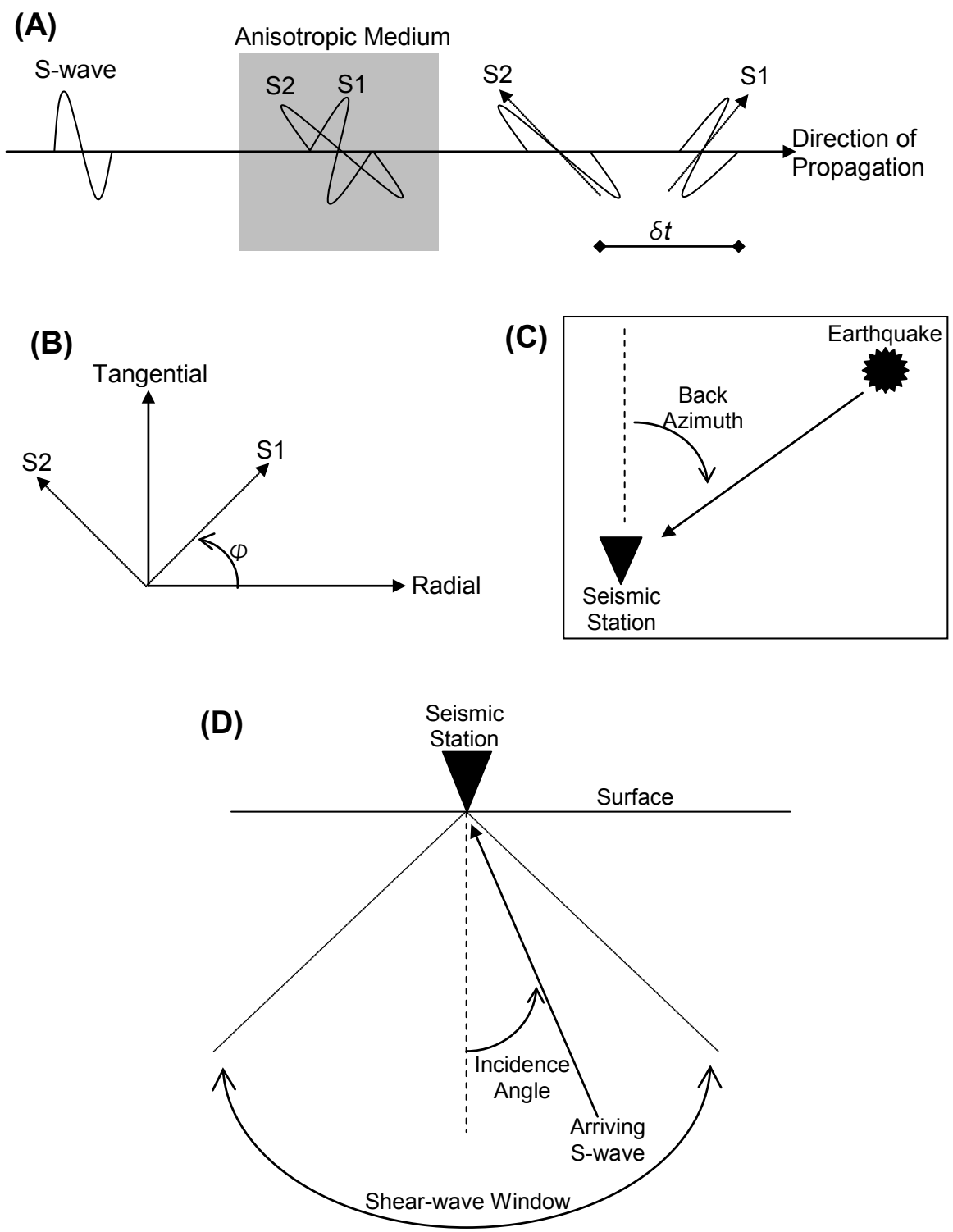

Figure 1.3 Diagrams of shear-wave splitting terminology. (A) Shear waves (S-wave) pasting through an anisotropic medium will be partitioned into two orthogonally polarized waves travelling at different velocities. The fast wave (S1) will be separated by a delay time $(\delta t)$ from the slow wave $(\mathrm{S} 2)$. Dashes arrows indicate polarization directions. (B) Polarization directions of S1 and S2 relative to the radial (event to station direction) and tangential (orthogonal to radial) directions. The fast wave is rotated by an angle $\varphi$ from the radial component, known as the fast direction. Herein, fast direction will be in reference to north. (C) Back azimuth is the angle from north between the seismic station and the earthquake event. (D) The incidence angle is the angle from vertical at which the shear wave energy is arriving at the station. The shear wave window is $45^{\circ}$ cone extending downward from the seismic station. 


\section{Data}

We used an earthquake catalog maintained by the University of Utah in which events are relocated utilizing three-dimensional (3-D) velocity models determined with seismic tomography (Husen et al. 2004) and probabilistic, non-linear earthquake location methods (Husen and Smith 2004). We examined earthquakes of location quality class "A", with an average location error less than 2.0-km, as defined by Husen and Smith (2004). Examined events were also required to have S-wave arrivals at incidence angles within the shear-wave window, more specifically less than $30^{\circ}$. Within the shear-wave window, waves are undistorted by interactions with any free surface or interface (Booth and Crampin 1985; Lou and Rial 1997). Incidence angles were determined by tracing rays through a 3-D velocity model (Husen et al. 2004) using the 3-D ray shooting algorithm implemented by Haslinger and Kissling (2001).

Earthquake event data were gathered from the Incorporated Research Institutions for Seismology (IRIS) Data Management Center (DMC) between January 2000 and August 2010 on nine three-component broadband stations located within and around Yellowstone caldera. See Table 2.1 for station details. A total of 9,556 arrivals were examined among the nine stations. 
Table 2.1

Seismic station information. Begin and end dates indicate the dates for which data was available during the 2000-2010 period of study. \#EQ indicates how many events for which data was collected on each station. \#Calc indicates how many events for which splitting parameters were calculated.

\begin{tabular}{|c|c|c|c|c|c|c|c|c|}
\hline Station & Network & Location & $\begin{array}{c}\text { Sample } \\
\text { Rate } \\
(\mathrm{Hz})\end{array}$ & Sensor & $\begin{array}{l}\text { Begin } \\
\text { Date }\end{array}$ & $\begin{array}{l}\text { End } \\
\text { Date }\end{array}$ & \#EQ & \#Calc \\
\hline B208* & PB & $\begin{array}{l}\text { Yellow- } \\
\text { stone Lake }\end{array}$ & 100 & $\begin{array}{l}\text { GeoSpace } \\
\text { HS-1-LT }\end{array}$ & $\begin{array}{l}\text { NOV } \\
2007\end{array}$ & $\begin{array}{l}\text { AUG } \\
2010\end{array}$ & 26 & 7 \\
\hline H17A & TA & $\begin{array}{l}\text { Grant } \\
\text { Village }\end{array}$ & 40 & $\begin{array}{l}\text { Streckheisen } \\
\text { STS-2 }\end{array}$ & $\begin{array}{l}\text { OCT } \\
2007\end{array}$ & $\begin{array}{l}\text { AUG } \\
2010\end{array}$ & 331 & 19 \\
\hline \multirow{2}{*}{ LKWY } & \multirow{2}{*}{ US } & \multirow{2}{*}{$\begin{array}{l}\text { Yellow- } \\
\text { stone Lake }\end{array}$} & \multirow{2}{*}{40} & $\begin{array}{c}\text { Guralp } \\
\text { CMG-3T }\end{array}$ & $\begin{array}{l}\text { JAN } \\
2000\end{array}$ & $\begin{array}{l}\text { OCT } \\
2004\end{array}$ & \multirow{2}{*}{363} & \multirow{2}{*}{32} \\
\hline & & & & $\begin{array}{l}\text { Streckheisen } \\
\text { STS-2 }\end{array}$ & $\begin{array}{l}\text { OCT } \\
2004\end{array}$ & $\begin{array}{l}\text { AUG } \\
2010\end{array}$ & & \\
\hline YFT & WY & $\begin{array}{l}\text { Old } \\
\text { Faithful }\end{array}$ & 100 & $\begin{array}{c}\text { Guralp } \\
\text { CMG-40T }\end{array}$ & $\begin{array}{l}\text { JAN } \\
2000\end{array}$ & $\begin{array}{l}\text { AUG } \\
2010\end{array}$ & 1087 & 166 \\
\hline YHH & WY & $\begin{array}{c}\text { Holmes } \\
\text { Hill }\end{array}$ & 100 & $\begin{array}{c}\text { Geotech } \\
\text { S-13 }\end{array}$ & $\begin{array}{l}\text { JAN } \\
2000\end{array}$ & $\begin{array}{l}\text { AUG } \\
2010\end{array}$ & 1624 & 630 \\
\hline YJC & WY & $\begin{array}{c}\text { Joseph's } \\
\text { Coat }\end{array}$ & 100 & $\begin{array}{l}\text { Geotech } \\
\text { S-13 }\end{array}$ & $\begin{array}{l}\text { JAN } \\
2000\end{array}$ & $\begin{array}{l}\text { AUG } \\
2010\end{array}$ & 365 & 74 \\
\hline YMP & WY & $\begin{array}{c}\text { Mirror } \\
\text { Lake } \\
\text { Plateau }\end{array}$ & 100 & $\begin{array}{l}\text { Geotech } \\
\text { S-13 }\end{array}$ & $\begin{array}{l}\text { SEP } \\
2002\end{array}$ & $\begin{array}{l}\text { AUG } \\
2010\end{array}$ & 2913 & 53 \\
\hline YMR & WY & $\begin{array}{c}\text { Madison } \\
\text { River }\end{array}$ & 100 & $\begin{array}{c}\text { Guralp } \\
\text { CMG-40T }\end{array}$ & $\begin{array}{l}\text { JAN } \\
2000\end{array}$ & $\begin{array}{l}\text { AUG } \\
2010\end{array}$ & 2059 & 424 \\
\hline YNR & WY & $\begin{array}{l}\text { Norris } \\
\text { Junction }\end{array}$ & 100 & $\begin{array}{c}\text { Guralp } \\
\text { CMG-40T }\end{array}$ & $\begin{array}{l}\text { JAN } \\
2000\end{array}$ & $\begin{array}{l}\text { AUG } \\
2010\end{array}$ & 788 & 160 \\
\hline
\end{tabular}

* B208 is a borehole station and buried at 161.3-m depth. 


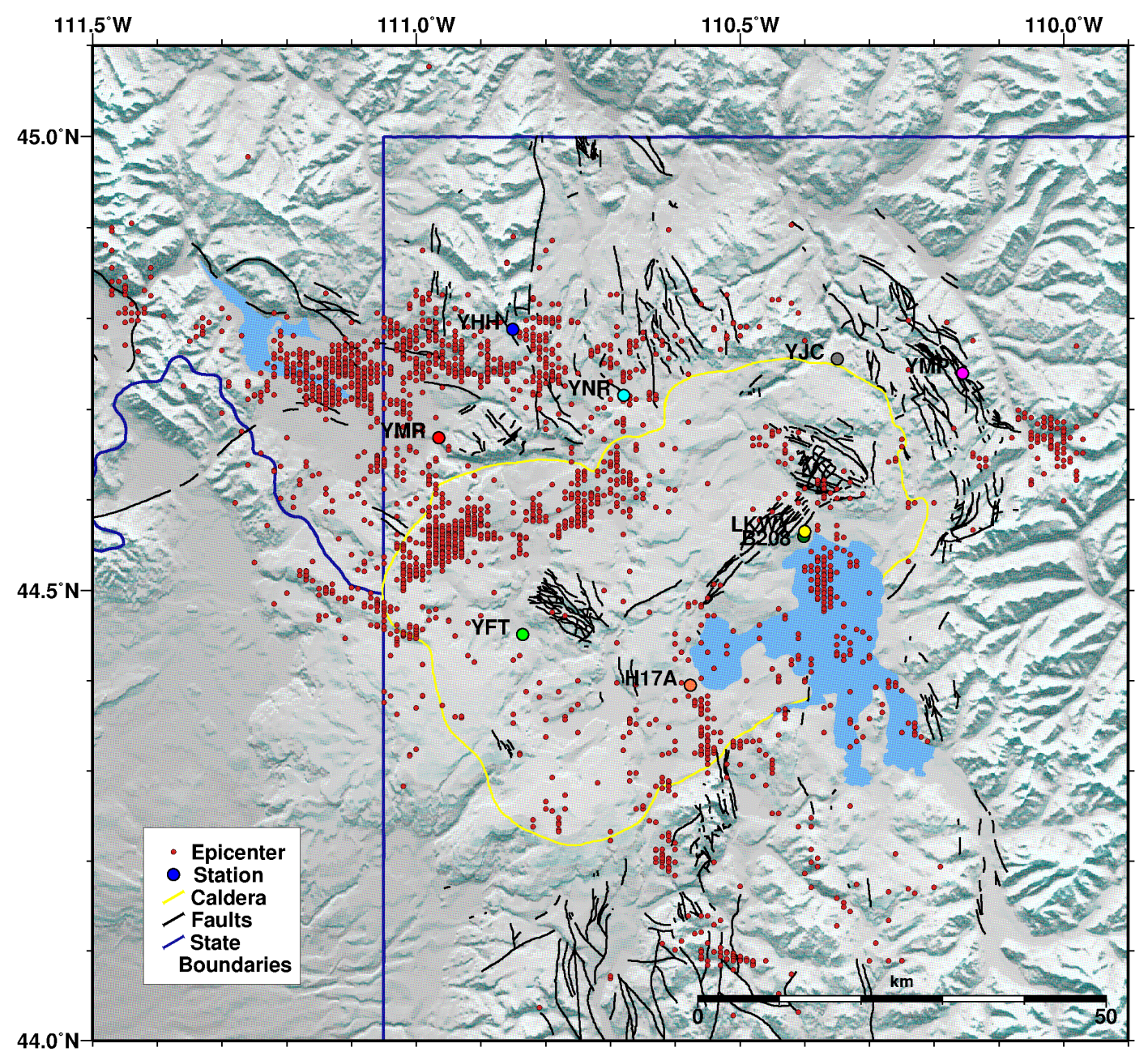

Figure 2.1 Earthquake and station location map. Epicenters indicate the location of earthquakes that fit the parameters of the study (quality "A," and $<30^{\circ}$ incidence angle) and for which data was available. The majority of earthquakes occur in the northwest corner of the caldera near stations YHH, YMR, and YNR. 


\section{Methodology}

\subsection{Shear-wave Splitting Parameter Calculations}

Splitting parameters were calculated using two different methodologies: Silver and Chan (1991) and Levin et al. (1999). The splitting parameter calculation algorithms were adopted and adapted from Waite et al. (2005).

The Silver and Chan (1991) method performs a grid search over possible $\varphi$ and $\delta t$ values and seeks a pair that effectively minimizes energy on the tangential component. In an isotropic medium, all energy will be concentrated on the radial component. Presence of energy on the tangential component indicates anisotropy. From the minimized transverse energy angle, fast directions can be determined. Uncertainty estimates are made using $F$ test statistics. This method was originally designed to estimate splitting parameters on teleseismic events using the SKS phase. As the method has been adapted for use on local earthquakes and S phase energy, the initial polarization direction, known in teleseismic events, becomes an additional unknown parameter that must be solved prior to splitting estimations.

The Levin et al. (1999) method also performs a grid search over possible splitting parameters, but seeks a pair that produces maximal similarity in shape of the two rotated horizontal waveforms as quantified by cross-correlation. Error estimates are calculated based on the curvature of the misfit function.

Ideally, the results of these two methods should correlate. The grid search values are detailed in Table 3.1. Selecting appropriate grid search values was achieved largely on a trial and error basis. Choosing the limits of $\varphi$ is straightforward as polarizations have a maximum variation of $180^{\circ}$. Choosing the limits of $\delta t$ is less certain. Allowing $\delta t$ to vary too little results in large delay times being misrepresented, and allowing $\delta t$ to vary too much results in increased processing time. The upper limit for $\delta t$ was set at $0.296 \mathrm{sec}$, which we believe to encompass the largest delay times in the data set. Grid search steps 
of $2^{\circ}$ for $\Delta \varphi$ and $0.008 \mathrm{sec}$ for $\Delta \delta t$ are small enough to account for slight changes in splitting parameters, yet keep processing time minimal.

\subsection{The Analysis Window}

Results are often sensitive to the choice of the shear-wave analysis window which is the time window over which splitting parameters will be determined. Ideally the analysis window should begin slightly before the arrival of the S-wave energy, contain several periods of the dominant frequency and little noise. These criteria help to stabilize results and reduce the effects of cycle skipping. However, rarely does this ideal present itself in the data. Realistically, we attempted to choose windows that included one period of the dominant frequency. Windows smaller than one period result in unreliable splitting estimates because only short fragments of the waveform need to be matched, and windows larger than one period begin to include secondary phases that decrease the quality of the splitting estimates. Noise in the data can also greatly affect the splitting estimates. Noise affects the ability to determine the exact arrival of S-wave energy at the station, to determine the end of a single period, and degrades the splitting estimates. Noisy signals can result in unrealistically large $\delta t$ due to cycle skipping effects (Vecsey et al. 2008).

In order to counteract the effects of user-bias in the selection of the analysis window, we automated splitting parameter estimates over a range of start and end times based upon an initial choice of the S-wave arrival. By limiting user-input to a single parameter, the approximate S-wave arrival, we eliminate biases in window selection in hopes of finding stability in a wide range of analysis windows. Also, by analyzing over a range of windows, we reduce the criticality of selecting the exact arrival of the S-wave energy. Each of the 9,556 arrivals was examined, and for events in which the S-wave arrival could be estimated confidently, arrival times were picked. A band-pass filter (0.5-19.5 $\mathrm{Hz}$ ) was applied solely for picking purposes in order to increase the signal to noise ratio. By filtering the data for picking, we significantly increased the portion of events for which arrival times could be picked. All attempts were made to pick arrival times 
slightly before the arrival of the S-wave energy at the station in order to ensure that the full phase energy would be included in parameter calculations. Noisy data, low amplitudes, and occasional missing horizontal channel data inhibited picking on the majority of events. Arrivals were picked on approximately $16 \%$ of events.

Splitting parameters were estimated for a range of analysis windows with varying begin and end times. The beginning of the analysis window $T_{b e g}$ is defined relative to the picked arrival time $T_{S}$ and varies by $N_{\text {beg }}$ steps of $\Delta T$. The end of the analysis window is defined relative to $T_{\text {beg }}$ and varies by $N_{\text {end }}$ steps of $\Delta T$. The shear-wave analysis window is defined by

$$
\begin{array}{ll}
T_{b e g}=T_{\mathrm{S}}+(i-5) \Delta \mathrm{T} & \text { for } \mathrm{i}=1 \ldots \mathrm{N}_{\text {beg }} \\
T_{\text {end }}=T_{\text {beg }}+(j+4) \Delta \mathrm{T} & \text { for } \mathrm{j}=1 \ldots \mathrm{N}_{\text {end }} .
\end{array}
$$

Window parameters are detailed in Table 3.1. The shortest analysis window was $0.25 \mathrm{~s}$, the minimum length of the dominant period, which varied between 0.2 and $0.25 \mathrm{~s}$. The largest analysis window was $0.65 \mathrm{~s}$, approximately 2.5 times the length of the dominant period. These window proportions were used by Savage et al. (2010).

Additionally, for each analysis window, splitting estimates were made after a two-pole Butterworth filter was applied for $B$ different frequency bands. Filtering served to progressively eliminate unwanted noise and study the stability of splitting estimates over different frequency bands. The total number of analysis windows for each event is given by $N=N_{\text {beg }} N_{\text {end }} B$.

\subsection{Cluster Analysis}

$N$ pairs of splitting parameters result from each event for each of the calculation methods. Simply selecting the window with the smallest errors as the best is not effective, because an unstable result, sensitive to small window changes, may be selected. The best selection would be a pair of values that is stable over a wide range of windows, contained 


\section{Table 3.1}

Time window analysis and grid search parameters. Band $1-$ Band $_{5}$ describe the lower and upper frequencies used in filtering data prior to splitting parameter determination.

\begin{tabular}{|c|c|}
\hline $\begin{array}{l}\text { Analysis Window } \\
\text { Parameters }\end{array}$ & Value \\
\hline$N_{b e g}$ & 6 \\
\hline$N_{\text {end }}$ & 9 \\
\hline$B$ & 5 \\
\hline Band $_{1}$ & $0.05-19 \mathrm{~Hz}$ \\
\hline Band $_{2}$ & $1-10 \mathrm{~Hz}$ \\
\hline $\mathrm{Band}_{3}$ & $2-7 \mathrm{~Hz}$ \\
\hline $\mathrm{Band}_{4}$ & $3-8 \mathrm{~Hz}$ \\
\hline \multirow[t]{2}{*}{ Band $_{5}$} & $4-10 \mathrm{~Hz}$ \\
\hline & 270 \\
\hline$\Delta T$ & $0.05 \mathrm{sec}$ \\
\hline $\begin{array}{l}\text { Grid Search } \\
\text { Parameters }\end{array}$ & Value \\
\hline$\varphi$ & $0-180^{\circ}$ \\
\hline$\Delta \varphi$ & $2^{\circ}$ \\
\hline$\delta t$ & $0-0.296 \mathrm{sec}$ \\
\hline$\Delta \delta t$ & $0.008 \mathrm{sec}$ \\
\hline
\end{tabular}

within cluster of similar measurements. We use the automated cluster analysis technique of Teanby et al. (2004) to cluster the data, select the optimum number of clusters, and ultimately select the optimum cluster and measurement. This methodology is detailed in Appendix A.

\subsection{Cycle Skipping}

Upon reviewing the results, it became apparent that many of the events' measurements were being affected by cycle skipping. If waveforms are mismatched by one-half cycle, the fast and slow waves can be interchanged, thus $\varphi$ would differ by $90^{\circ}$ and $\delta t$ by onehalf period (Matcham et al. 2000). If the waveforms are mismatched by an entire cycle, $\varphi$ remains the same, but $\delta t$ will differ by a whole period (Savage et al. 2010). In the 
splitting measurements, clusters at $\delta t$ differing by half and whole periods were clearly discernable (Fig. 3.1).

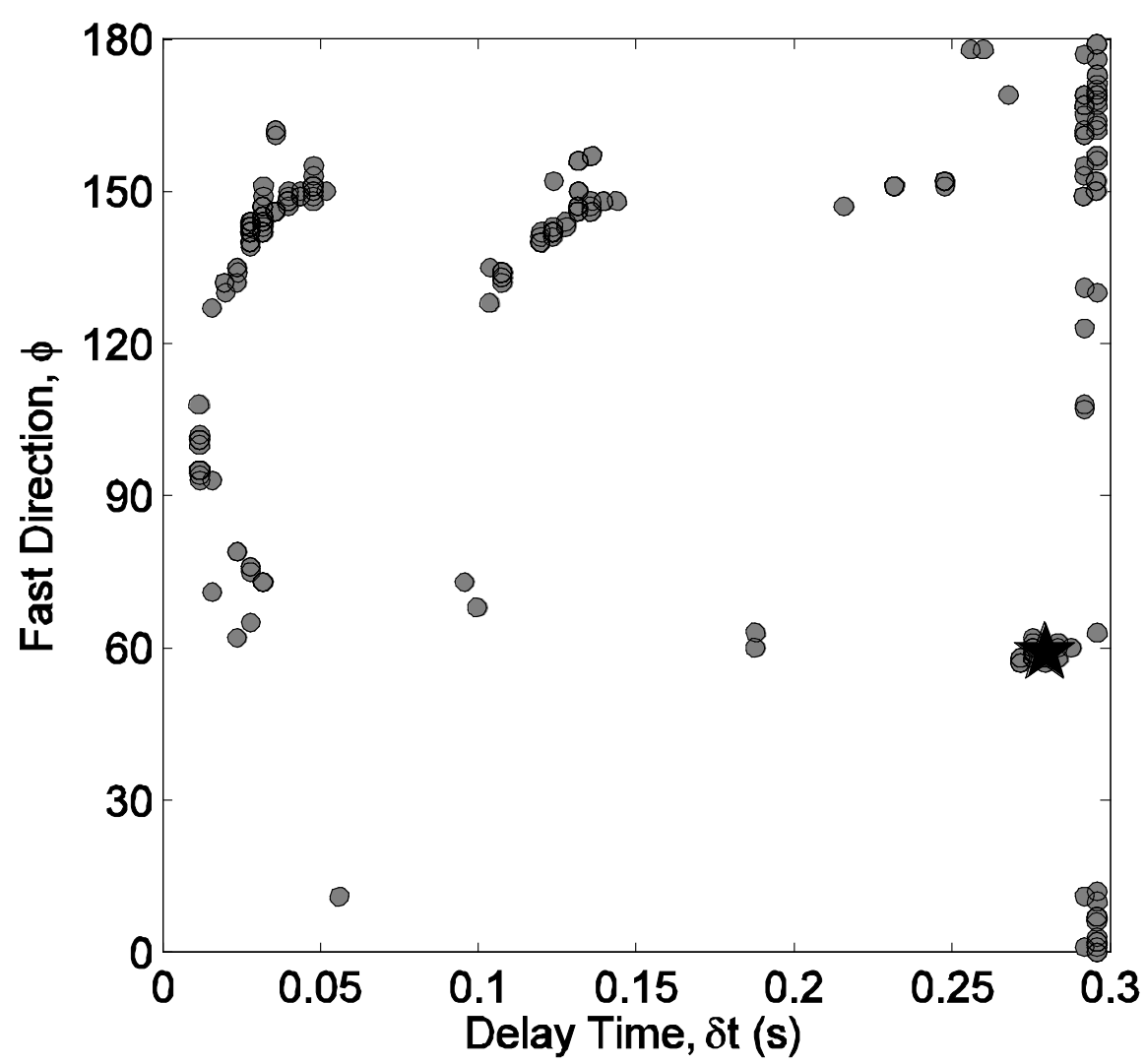

Figure 3.1 Visible effects of cycle skipping. This plot shows the splitting parameters estimated for a single event (gray dots), and the optimum measurement chosen by the automated cluster analysis (black star). Several distinct clusters are visible at delay times differing by half-periods $(0.04 \mathrm{~s}, 0.14 \mathrm{~s}, 0.24 \mathrm{~s}$, and near $0.3 \mathrm{~s})$ and two orthogonal fast polarization directions $\left(\sim 60^{\circ}\right.$ and $\left.\sim 150^{\circ}\right)$. The spread of these splitting estimates is characteristic of an event that has been affected by cycle skipping. In this example, the optimum measurement is automatically chosen at $60^{\circ}, 0.27 \mathrm{~s}(\varphi, \delta t)$, whereas a more optimum measurement would likely be chosen at $150^{\circ}, 0.04 \mathrm{~s}$ had cycle skipping not been a factor.

In order to neutralize the effects of cycle skipping, we manually defined three time bins that corresponded to the clearly discernable clustering at half cycle increments

(Table 3.2). Within each bin, on each event, we calculated the percentage of $N$ splitting estimates occurring. If, on a single event, more than a specified percentage $P_{\max }$ of the 
Table 3.2

Cycle skipping parameters.

\begin{tabular}{|l|l|}
\hline $\begin{array}{l}\text { Cycle Skipping } \\
\text { Parameters }\end{array}$ & Value \\
\hline Time Bin 1 & $0-0.09 \mathrm{sec}$ \\
\hline Time Bin 2 & $0.09-0.19 \mathrm{sec}$ \\
\hline Time Bin 3 & $0.19-0.296 \mathrm{sec}$ \\
\hline$P_{\max }$ & $20 \%$ \\
\hline
\end{tabular}

splitting estimates fell into more than one time bin, the event was flagged as being affected by cycle skipping. We assumed that due to the local nature of the earthquakes and thus short path lengths, generally $\delta t$ is expected to have lower values. If an event was identified as being affected by cycle skipping, all measurements beyond the maximum $\delta t$ defining the smallest time bin containing more than $P_{\max }$ of the measurements were eliminated, and the automated cluster analysis was performed again on the reduced data set. For example, if an event was flagged for cycle skipping, and more than $20 \%$ of the measurements fell into bins 2 and 3, measurements beyond $0.19 \mathrm{sec}$ would be eliminated prior to redoing the cluster analysis. The $20 \%$ cutoff was defined through experimentation. Choosing a lower percentage resulted in nearly all events being flagged for cycle skipping, and while there is a significant amount of scatter in the $N$ measurements for each of the events, not all optimum measurements are affected by the scattering. Choosing a lower percentage can alter optimum measurements on events for which the optimum choice is already accurate and also affect optimum measurements on events that legitimately have larger $\delta t$ values. Choosing a higher percentage resulted in fewer events being flagged for cycle skipping and its effects negatively impacted the results.

By reducing the effect of cycle skipping, we partially impede optimum measurements being chosen at unrealistically large $\delta t$ and improve the quality of our automatically chosen splitting estimates. Despite our efforts, several events are still represented by large $\delta t$. In these events, cycle skipping was not identified, and the original optimal measurements were maintained. It is possible that these large $\delta t$ values accurately represent the degree of splitting and indicate a longer path through the anisotropic 
medium. It is also possible that the majority of the $N$ measurements on these events resulted from cycle skipping, and more than $20 \%$ of the measurements did not fall into more than one time bin, thus the event was not flagged. 


\section{Results}

Of the 9,556 S-wave arrivals examined, 1,565 arrivals (approximately 16\%) were picked and analyzed.

\subsection{The Silver and Chan (1991) Method}

The optimum splitting parameters estimated by the Silver and Chan (1991) method and chosen by the automated cluster analysis are presented in Figure 4.1. From this map we can see that there is a great degree of variance in $\varphi$ at each of the stations and a propensity for large $\delta t$. With the abundance and variance of information on this map, it is difficult to discern definite trends. On the stations with fewer arrivals, such as LKWY, YJC, and YMP, we can clearly see orthogonally natured splitting measurements, some oriented North-South and some oriented East-West. Stations YFT, YHH, YMR, and YNR have widely variable results. Trends in the data are more clearly detectable in Figure 4.2 which displays the splitting results for each station as rose plots. Stations B208, and H17A have too few arrivals to demonstrate any clearly defined trends. The remaining seven stations, however, each exhibit apparent preferences for fast direction. Stations LKWY, YJC, and YMP show a preference for generally N-S fast directions, and stations YFT, YHH, YMR, and YNR show a preference for more NW-SE oriented fast directions. Also visible in this figure are less major fast direction preferences perpendicular to the major preferences. Stations YFT and YMR prominently display this phenomenon with several events displaying NE-SW fast directions.

These orthogonally oriented fast directions were interpreted as effects of cycle skipping. Figures 4.3 - 4.6 show how cycle skipping can affect the optimum measurement chosen by the cluster analysis. Figure 4.3 shows four distinct $\delta t$ zones at $0.04 \mathrm{~s}, 0.11 \mathrm{~s}, 0.17-0.2$ $\mathrm{s}$, and $0.296 \mathrm{~s}$ into which the majority of the splitting estimates fall. In this case, the automated cluster analysis chose the optimum measurement at $129^{\circ}, 0.112 \mathrm{~s}(\varphi, \delta t)$. This splitting estimate does not result in strong correlation in the corrected waveforms or linear particle motion. Once the cycle skipping has been corrected (Fig. 4.4), the 
optimum measurement is chosen at $134^{\circ}, 0.036 \mathrm{~s}$ and the corrected waveforms match more closely while and the particle motion is linear. In this example, correction for cycle skipping did not involve a drastic change in $\varphi$; however, this is not the case for all events. Figure 4.5 displays how cycle skipping can affect both $\varphi$ and $\delta t$ measurements. Again, we can clearly see that the splitting estimates have clustered at distinct zones of $\delta t$, and the optimum measurement is chosen at $39^{\circ}, 0.28 \mathrm{~s}$. This measurement does result in a semi-linear particle motion, but the corrected waveforms do not correlate. After the cycle skipping has been corrected for and the optimum measurement is chose as $167^{\circ}, 0.052 \mathrm{~s}$ (Fig. 4.6), the corrected waveforms match much better while maintaining semi-linear

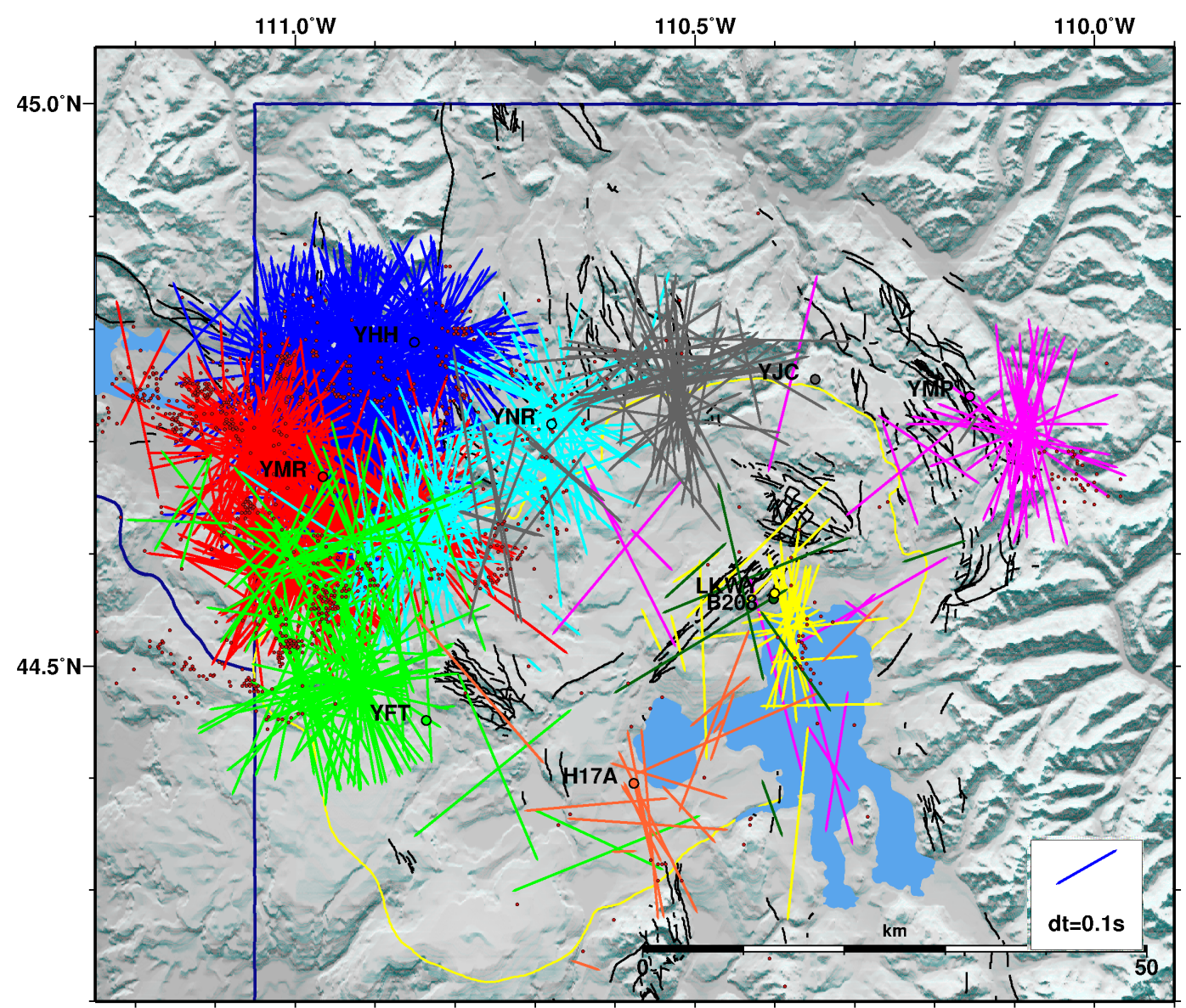

Figure 4.1 Shear wave splits resultant from the Silver and Chan (1991) method. The blue lines mark state boundaries. The black lines mark Quaternary faults. The yellow line demarcates the caldera rim. The sticks, color coded by station, indicate shear wave splits plotted halfway between the event (small red dots) and the station (larger colored dots). Orientation of the sticks corresponds to fast polarization direction $(\varphi)$ and length is proportional to the delay time $(\delta t)$. There is quite a bit of scatter in the data and thus trends are difficult to discern. 


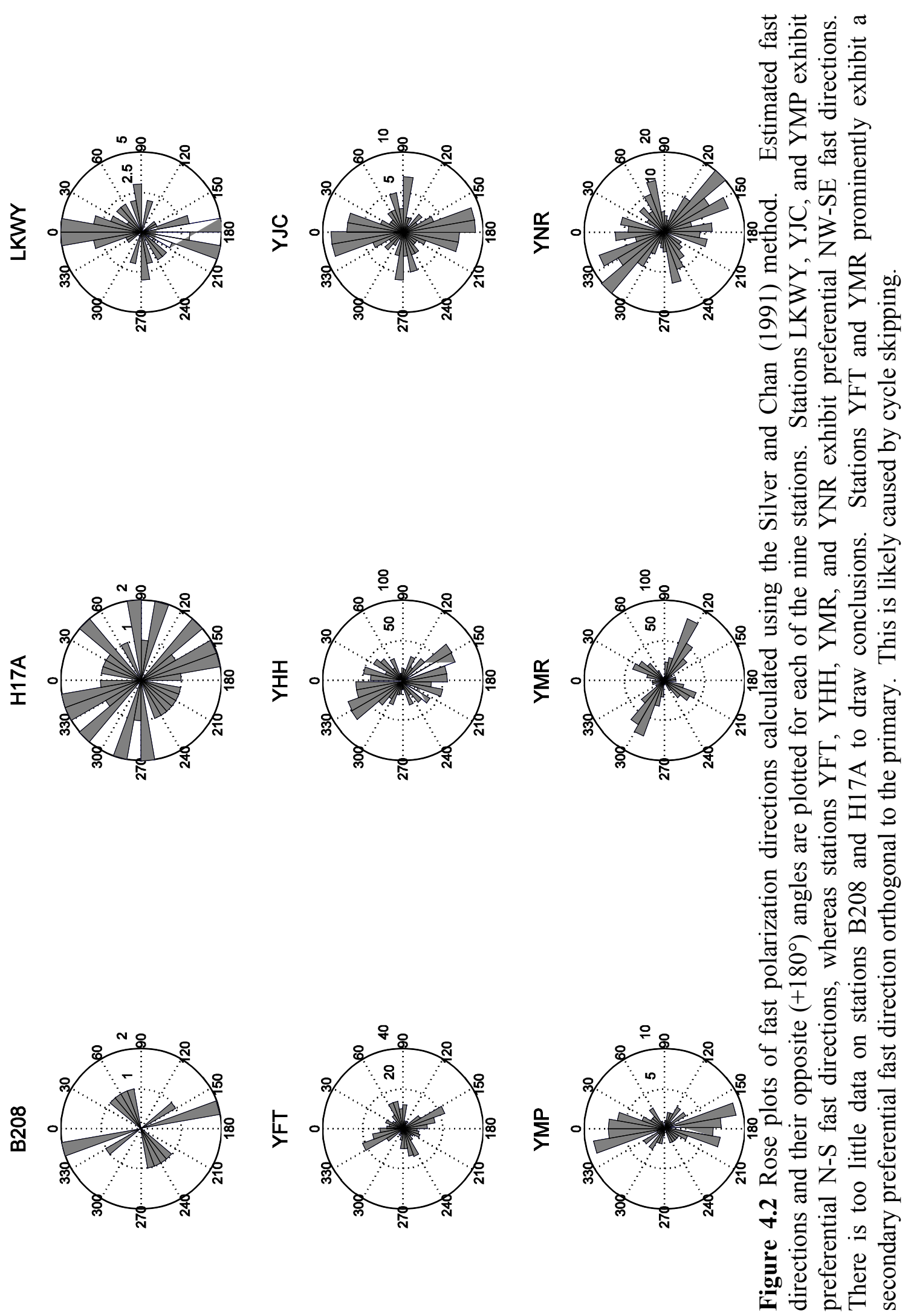




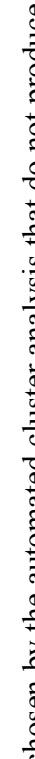

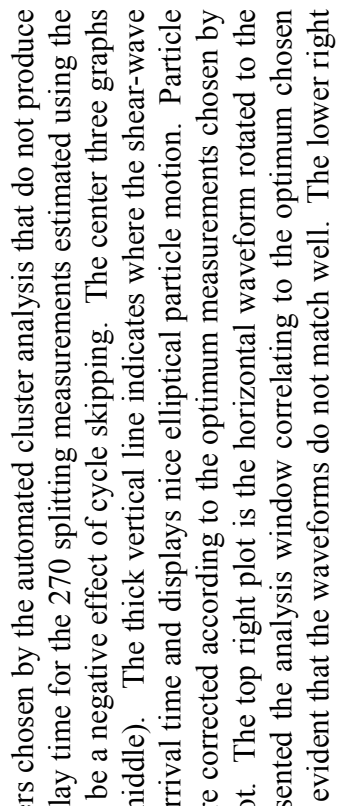

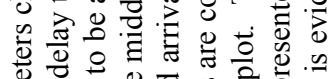
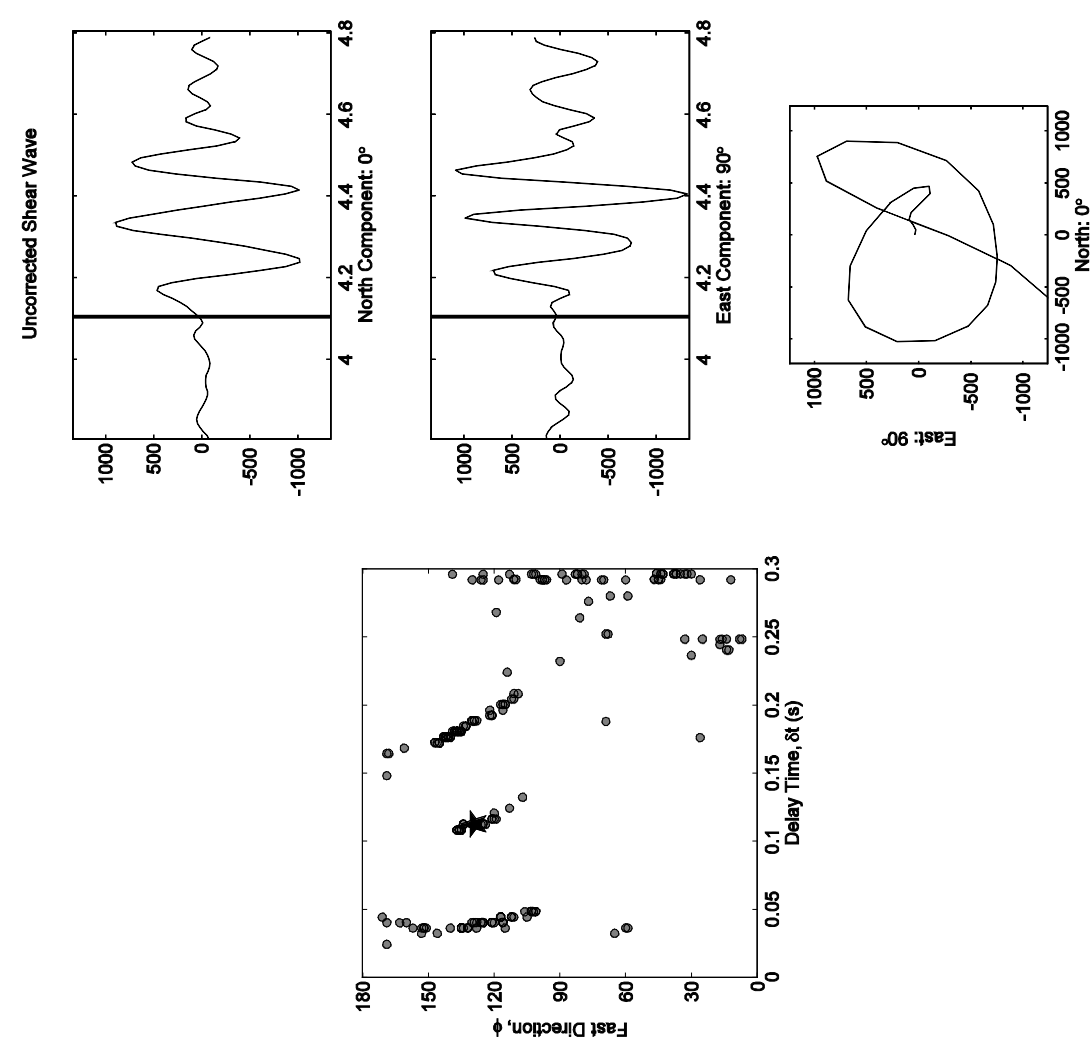

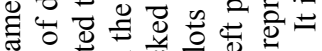

현

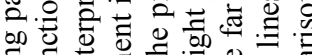

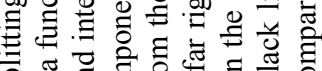

की

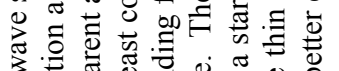

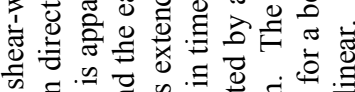

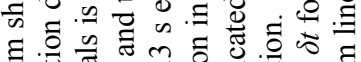

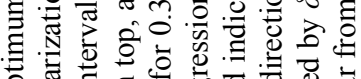

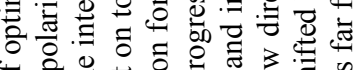

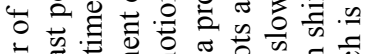

.

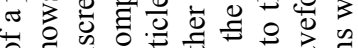

论过包

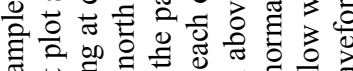

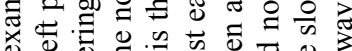

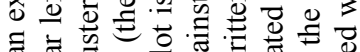

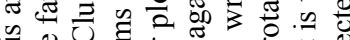

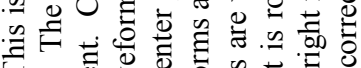

F.

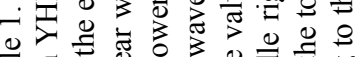

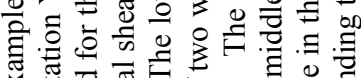

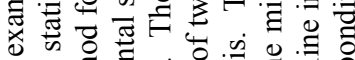

.

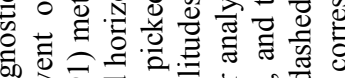

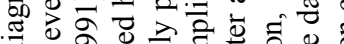

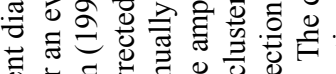

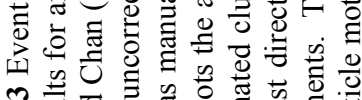

๗.

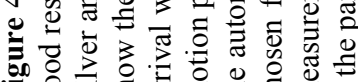



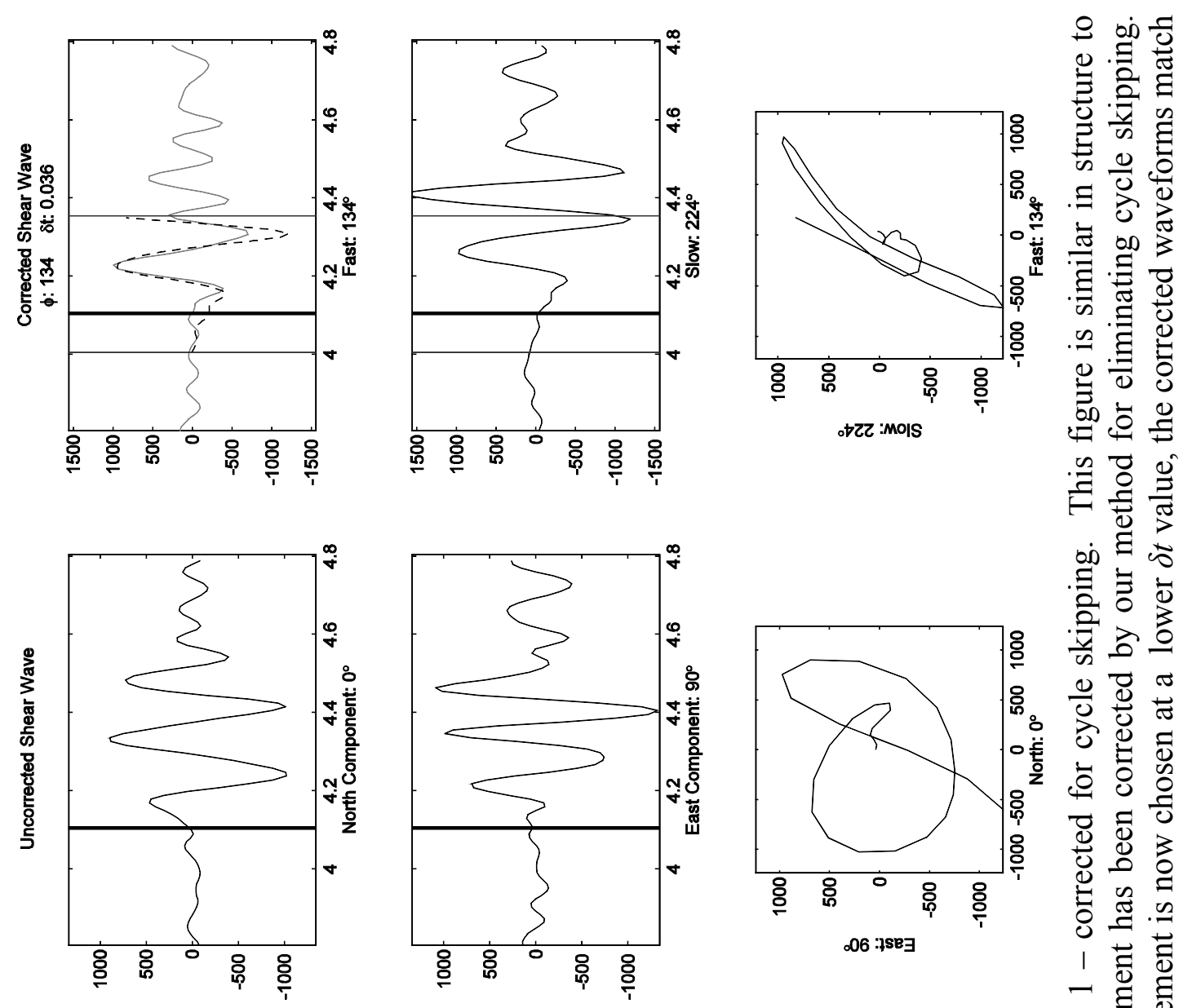

产总 $\frac{0}{0}$ को

寻

츤 으

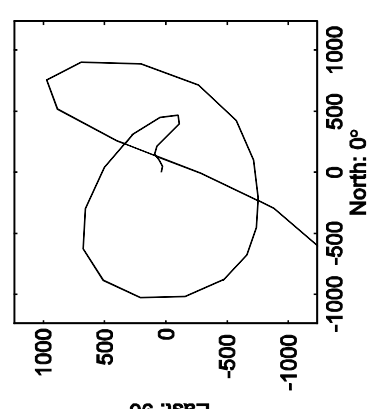

.06 :7Se

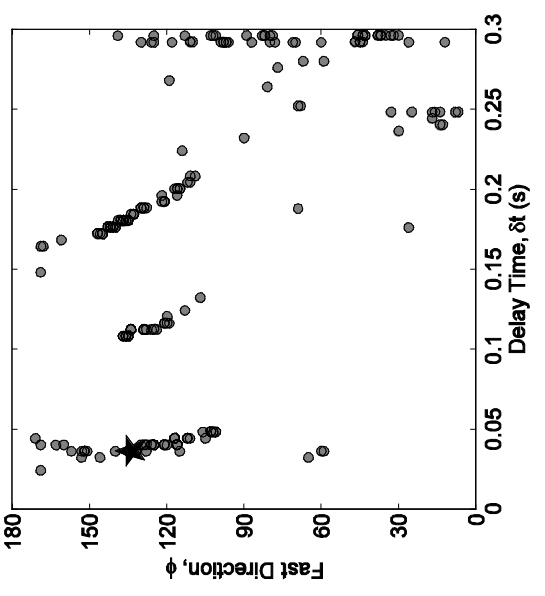

융

टे ญ

๘

웡

过

흥 胥.

1 芯芯

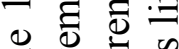

呈

苞 导

ฮ छ

-。号㤩元

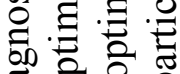

.ত্টة 000

号乒

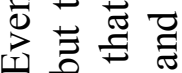

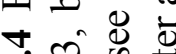

$\forall$ ?

๑ 0 ह

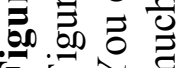



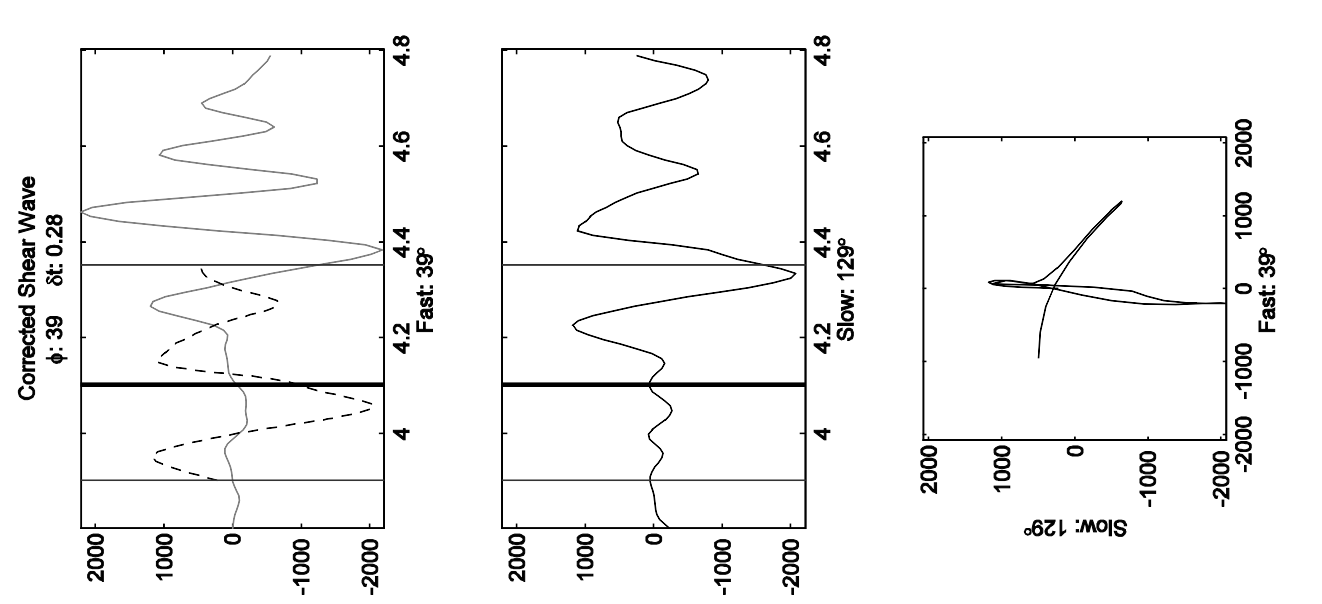

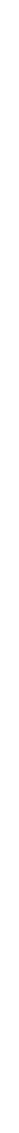

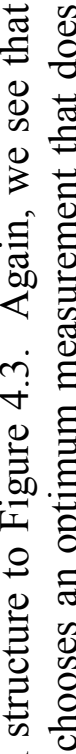
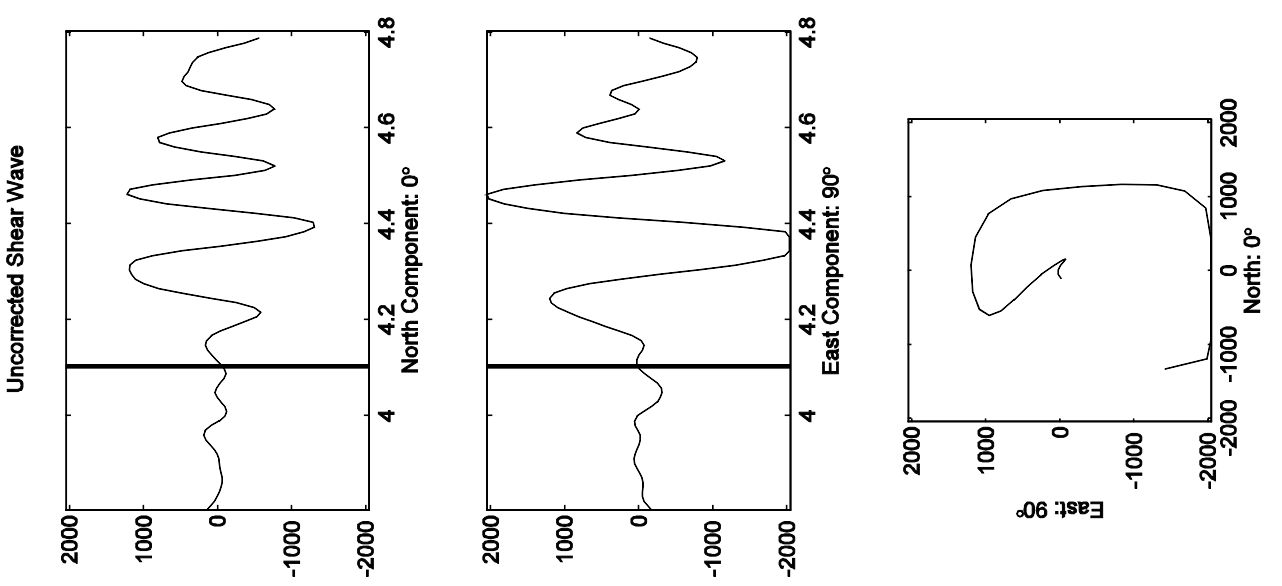

$\Xi$

해 $\frac{\pi}{\pi}$

的

.

告

先

त

을

䒕

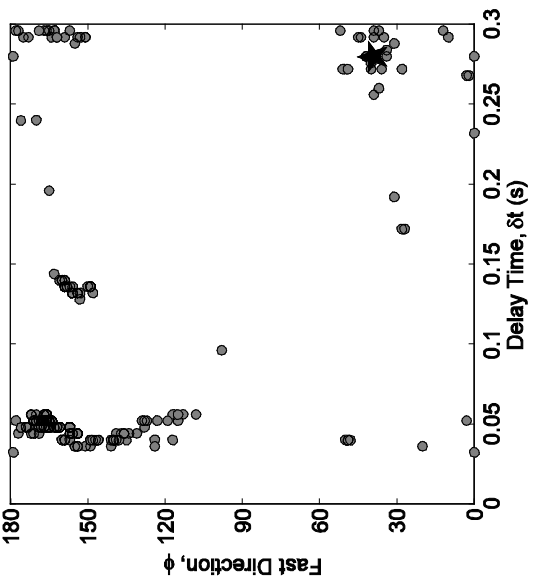

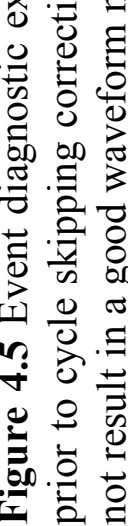



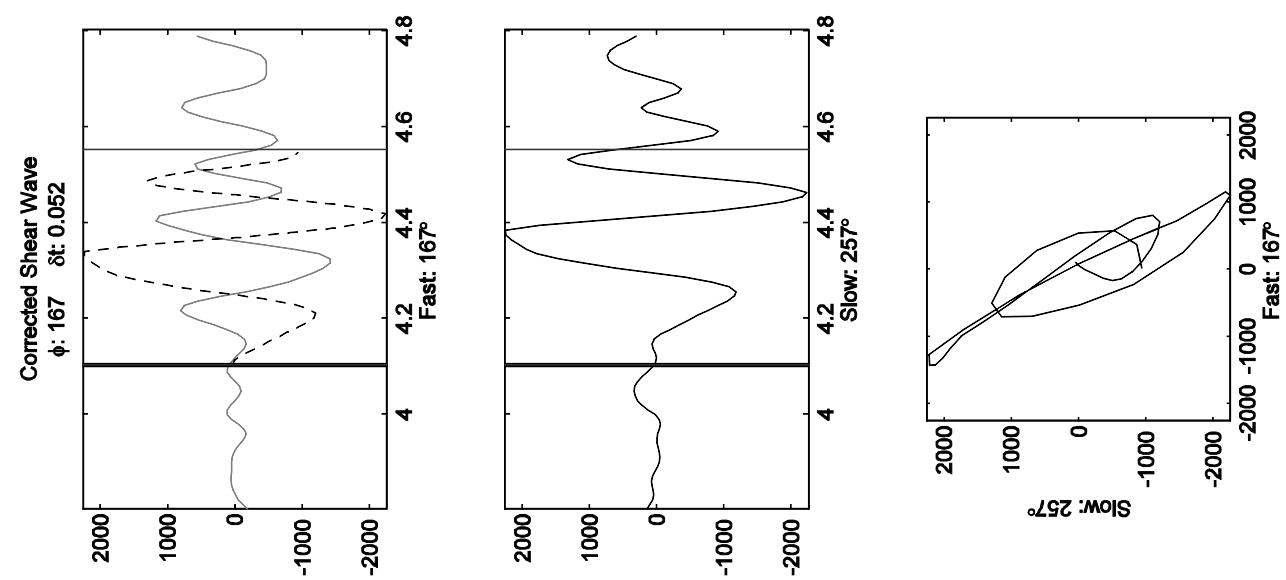

品

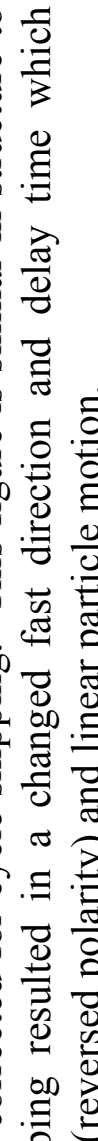
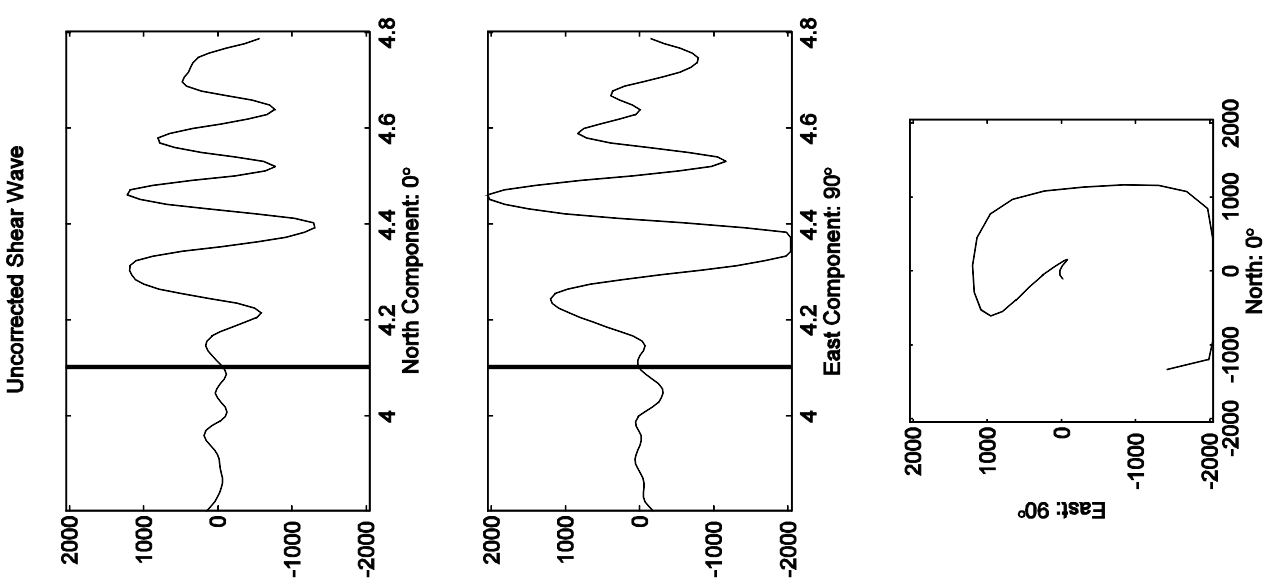

ㄱ. 를

을

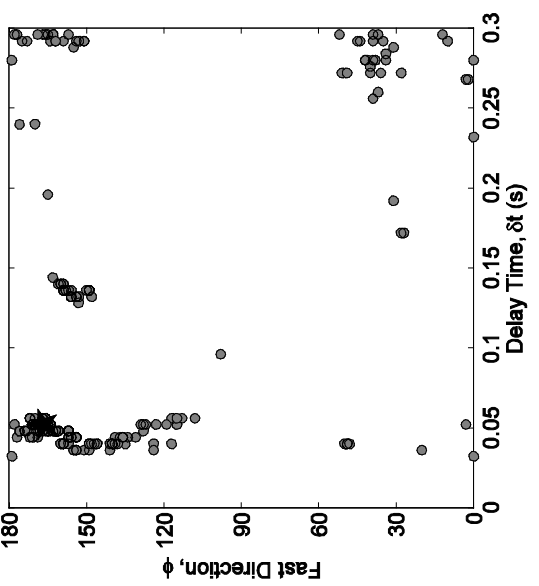

离

.

을

을

䒕记

II 잉

๒ $\dot{\nabla}$

를

些些 
particle motion.

Cycle skipping was automatically detected and solved for as detailed in the methodology, and the results are presented in Figures 4.7 and 4.8. While Figure 4.7 presents a clearer picture than Figure 4.1, and the majority of the high $\delta t$ measurements have been reduced, it is still difficult to discern trends due to the large amount of data. Figure 4.8 presents the information in a clearer manner again as rose plots for each station.

Stations YHH, YJC, YMR, and YNR maintain the preference for a N/NW-S/SE fast direction, but in a much more definitive manner reducing the number of scattered measurements. Station YFT exhibits a NE-SW fast direction preference which is orthogonal to the direction preference presented prior to corrections indicating that the majority of the events on the station were affected by cycle skipping. Station YMP, while still showing a NW-SE fast direction preference, does so less definitively. Finally, LKWY has diminished fast direction preference.

\subsection{The Levin et al. (1999) Method}

Although the Silver and Chan (1991) method was the primary method used in this study, we also used the Levin et al. (1999) method for determining shear-wave splitting parameters. These results are presented in Figure 4.9. While station B208 still lacks trend definition due to few arrivals, station H17A displays fast direction preferences at nearly orthogonal $130^{\circ}$ and $190^{\circ}$. Stations LKWY and YMP exhibit similar N-S preferential fast direction to those found in the Silver and Chan (1991) method. Station YHH shows a $30^{\circ}$ clockwise rotation of preferred fast direction. The secondary preferred direction on station YMR has rotated $30^{\circ}$ counter clockwise, and is not orthogonal to the primary preferred direction. Stations YFT, YJC, and YNR have scattered data and the preferential fast direction is more ambiguous as compared to the Silver and Chan (1991) method. 
Differences between the two methods could possibly be caused by noise in the data. The Levin et al.(1999) method relies on similarity in rotated waveforms to determine the splitting parameters. However, with noisy data, it becomes increasingly difficult to find the likeness, and best cross-correlation coefficients may not accurately represent the best splitting estimates. Differences between the methods could also arise due to the cluster analysis technique. It was evidenced from looking at the splitting parameters on individual events that the Levin et al. (1999) method estimates are hindered by large groupings at unrealistically low $\delta t$ rather than high $\delta t$ that affect the Silver and Chan (1991) method estimates, and the technique may be unsuited for handing this type of grouping.

The cycle skipping elimination method was also utilized for the Levin et al. (1999) method and the results are presented in Figure 4.10. Once again, significance cannot be gleaned from station B208. Station H17A exhibits increased definition at its preferred fast directions while definition on the other seven stations diminishes. This deterioration is most likely resultant from the cycle skipping elimination method being designed specifically with the SC data in mind and is therefore not applicable to the Levin et al. (1999) method.

Due to the differences in splitting parameter results for the two methods and the cycle skipping customization for the Silver and Chan (1991) method, these data corrected for cycle skipping were used for further investigation. 


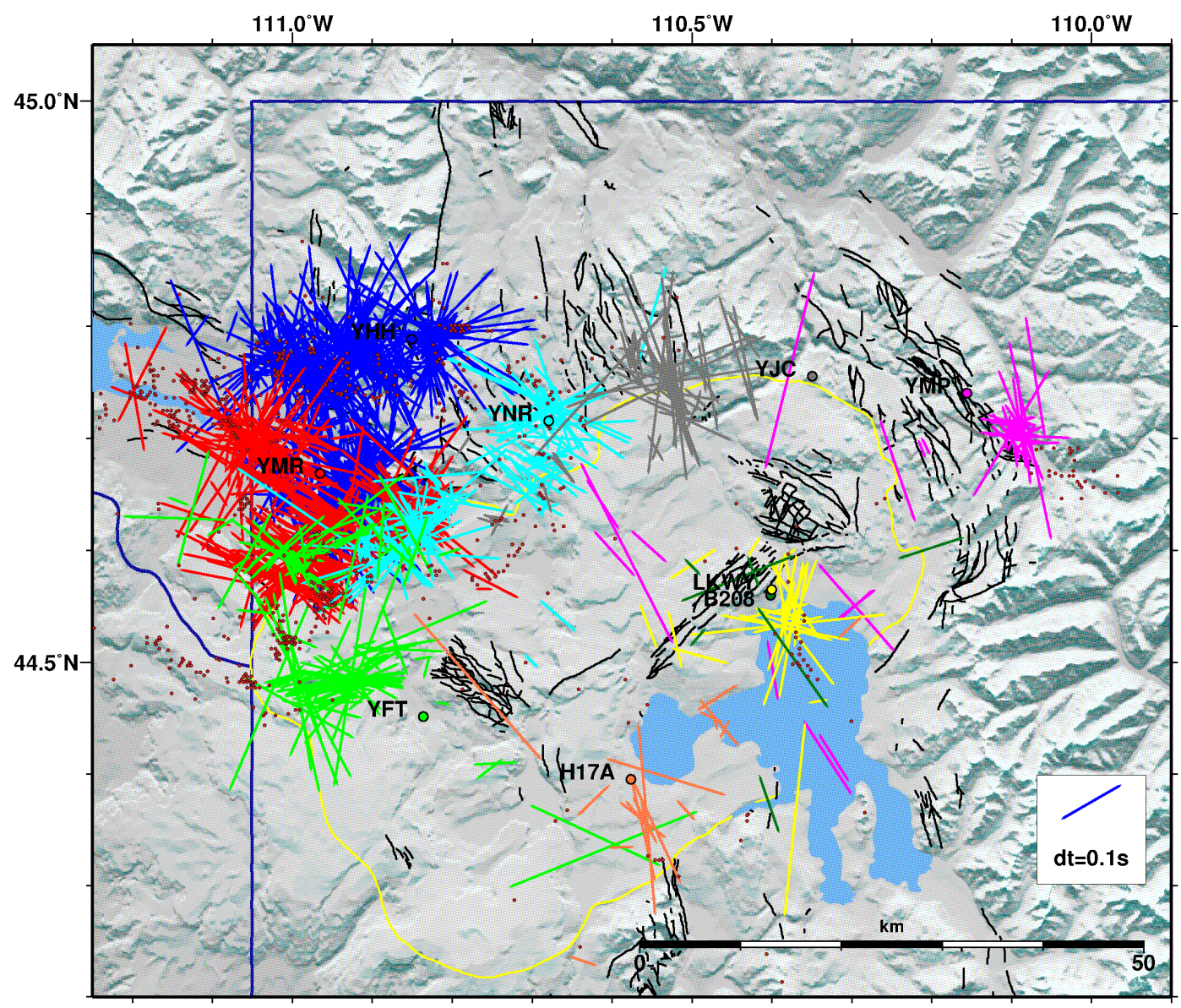

Figure 4.7 Shear wave splits resultant from the Silver and Chan (1991) method corrected for cycle skipping. Similar in structure to Figure 4.1, this map exhibits cleaner looking results likely due to the decrease in number of large $\delta t$ caused by cycle skipping, but definitive trends are still difficult to see. 


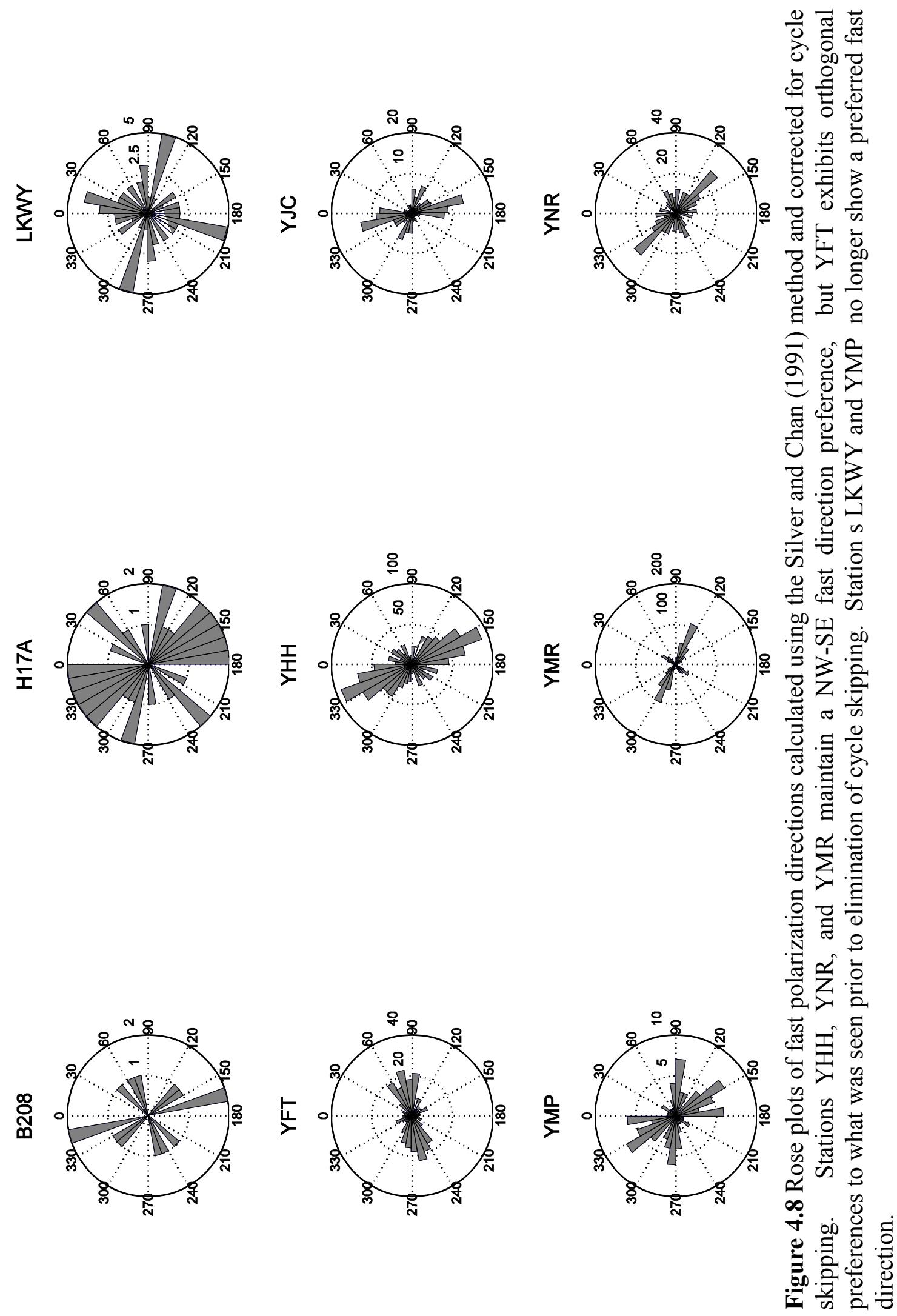




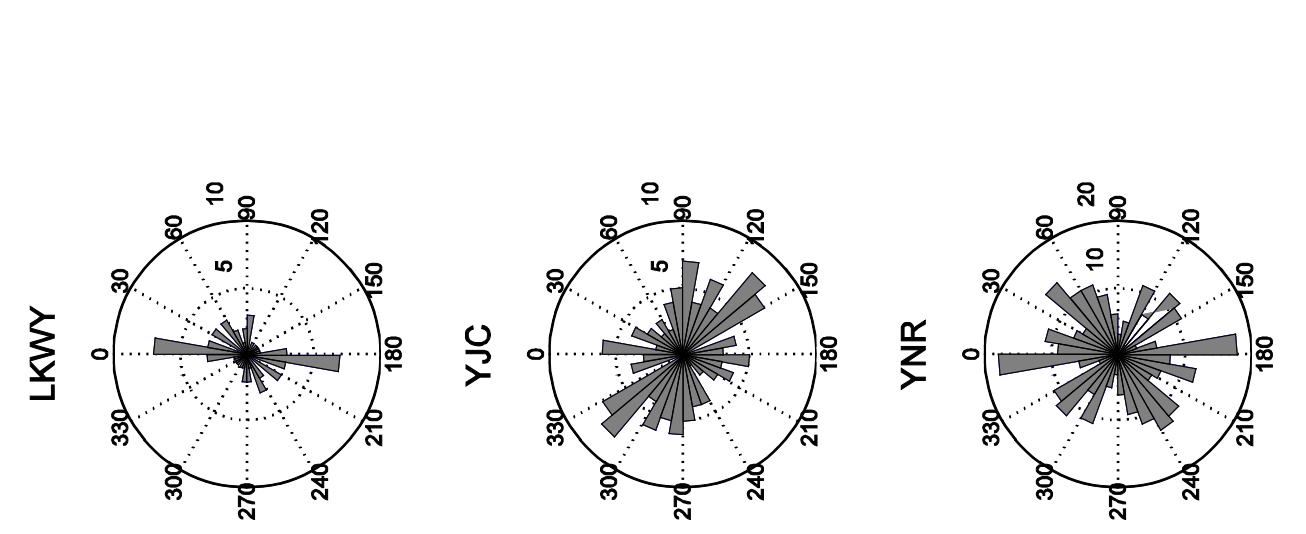

혼. 욜

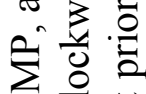

$\searrow 0$

武官宅

焉

墕

की 羟

ठ். 옹 옹

苞言

ลิ泀

은

宁震

एक

空焉
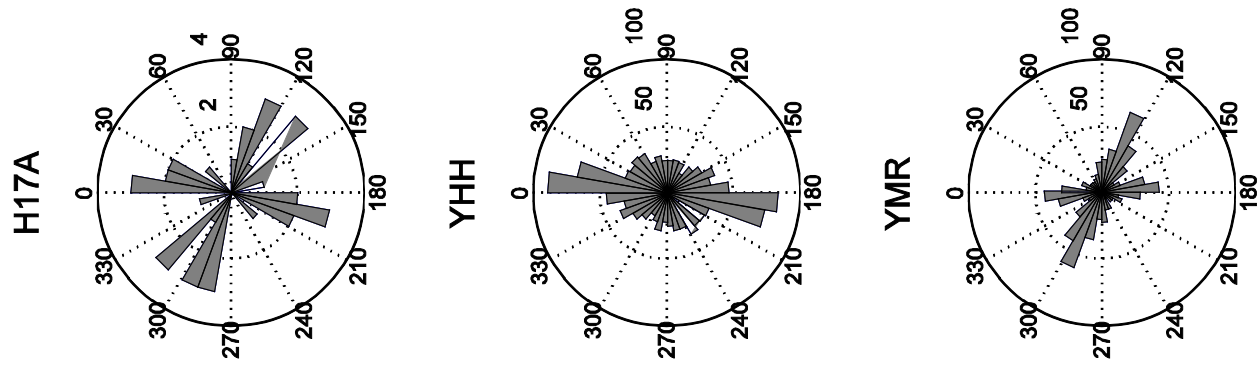

욜

कo

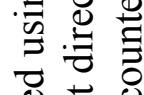

氖

离离

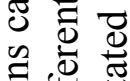

뉸

过

.

ธิ

啳

클
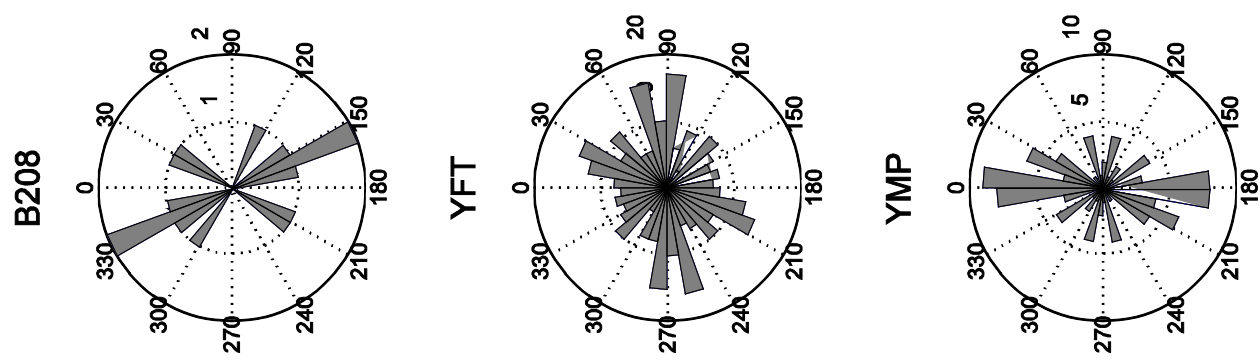

웡. 을

需

㟧 气. :

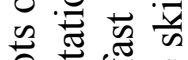

을 至

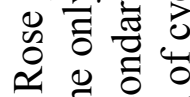

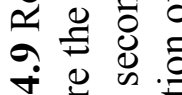

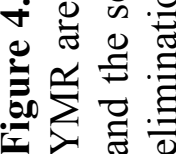




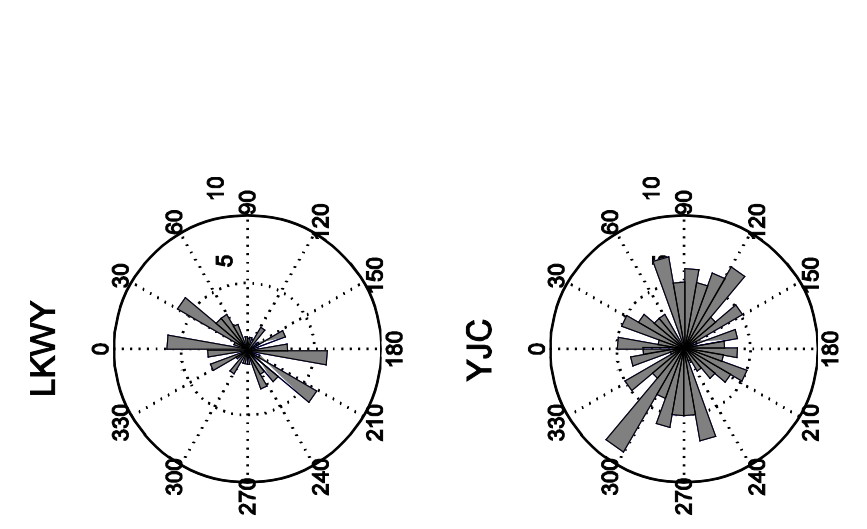

(1)
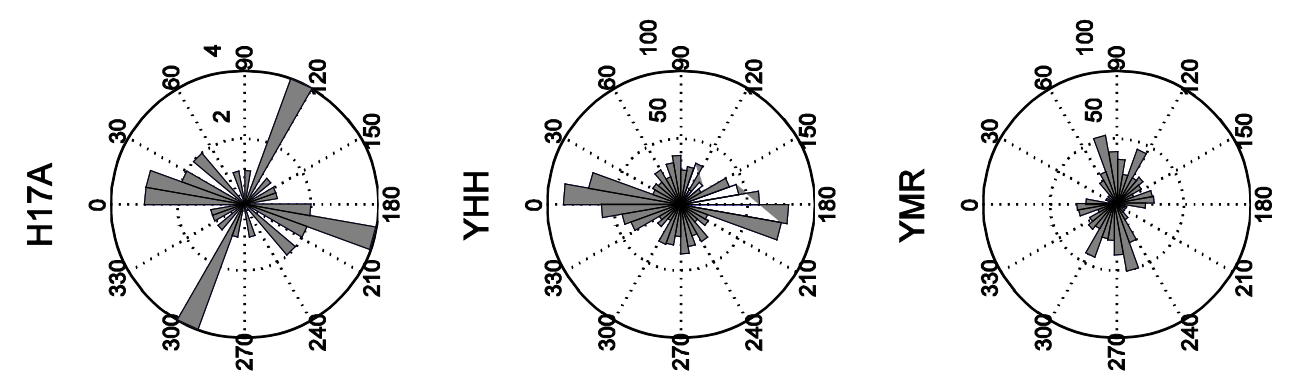

बूँ

तं

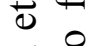

. 우

选

$\stackrel{ \pm}{ \pm}$

op $=$

$\exists . \Xi$

兽

.

可

常

을

芯

츨
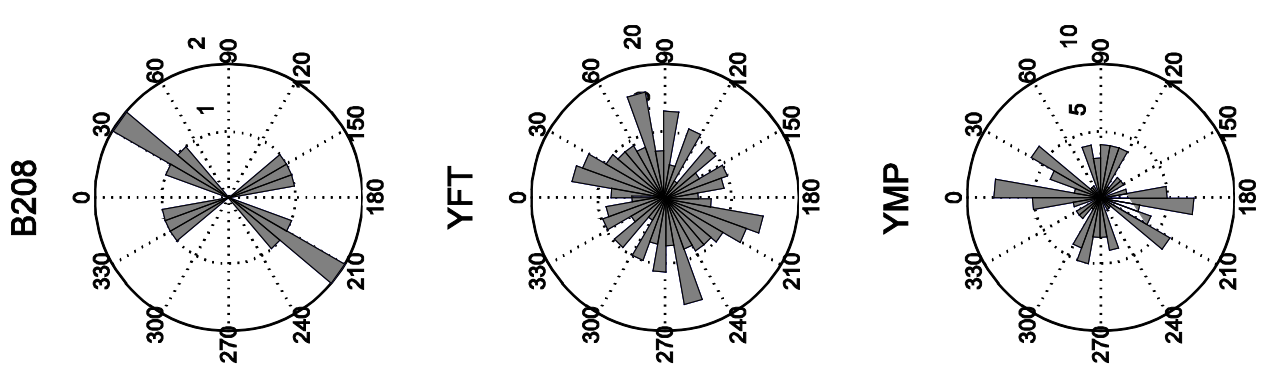

营. 产

는 $\approx \frac{0}{0}$

进 웜

늘

$\checkmark$ iो

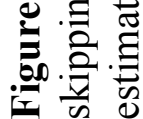




\section{Discussion}

There is a great deal of scatter in the shear-wave splitting estimates. Possible sources of scatter include noise in the data, improper shear-wave analysis window selection, remnant effects of cycle skipping, and improper incidence angle calculation. The incidence angles were calculated by shooting rays through a 3-D velocity model. It is possible that imperfection in the velocity model may have resulted in incorrect incidence angles. It is also possible that many correctly calculated incidence angles lie at edge of the shear wave window. Fast polarization directions on events with incidence angles near the edge of the shear-wave window can be rotated up to $90^{\circ}$ (Peacock et al. 1988; Savage et al. 2010) which may cause the orthogonally-oriented minor fast directions preferences and stations such as YMR. However, we identified no change in fast direction preferences at higher incidence angles. In addition to the features discussed below, we examined fast direction as a function of event distance and event depth, but were unable to define any trends.

\subsection{Comparisons to the Local Stress Field}

The local stress field around Yellowstone differs from the regional stress field dominated by NE-SW extension due to caldera deformation. Maximum extensional strain $\left(\varepsilon_{\max }\right)$ directions estimated from GPS measurements (Smith et al. 2009) and minimum principle stresses $\left(\sigma_{3}\right)$ estimated from focal mechanisms (Waite and Smith 2004) rotate from N-S NW of Yellowstone Caldera near the site of the 1959 M7.5 Hebgen Lake earthquake to NE-SW near the rim and within the caldera (Waite et al. 2005) (Figure 5.1). The calculated fast directions are nearly perpendicular to or significantly deviated from $\varepsilon_{\max }$ and $\sigma_{3}$ at YFT, YHH, YJC, YMP, YMR, and YNR as detailed in Table 5.1.

Waite and Smith (2004) and Waite and Chang (2007) invoke fluid-filled stress-oriented microcracks in the crust to explain these directions. This stress dependent anisotropy is created when cracks with faces oriented perpendicular to the maximum compressive 
stress $\left(\sigma_{\mathrm{H}_{\max }}\right)$ close, and cracks with faces oriented perpendicular to $\sigma_{3}$ open. In this situation, the fast direction is sub-parallel to the crack alignment and $\sigma_{\mathrm{H}_{\max }}$ (Crampin and Booth 1985; Savage et al. 1989) (Figure 5.2). If no deviations from the regional stress field existed, fast directions at all stations would be $\sim 151^{\circ}$, perpendicular to the direction of maximum horizontal extension parallel to the North American plate motion at $241^{\circ}$. The deviations of the stress field and corresponding fast directions at Yellowstone from the regional stress field indicate that local stresses derived from the deformation of the Yellowstone caldera are overprinting the regional stress field.

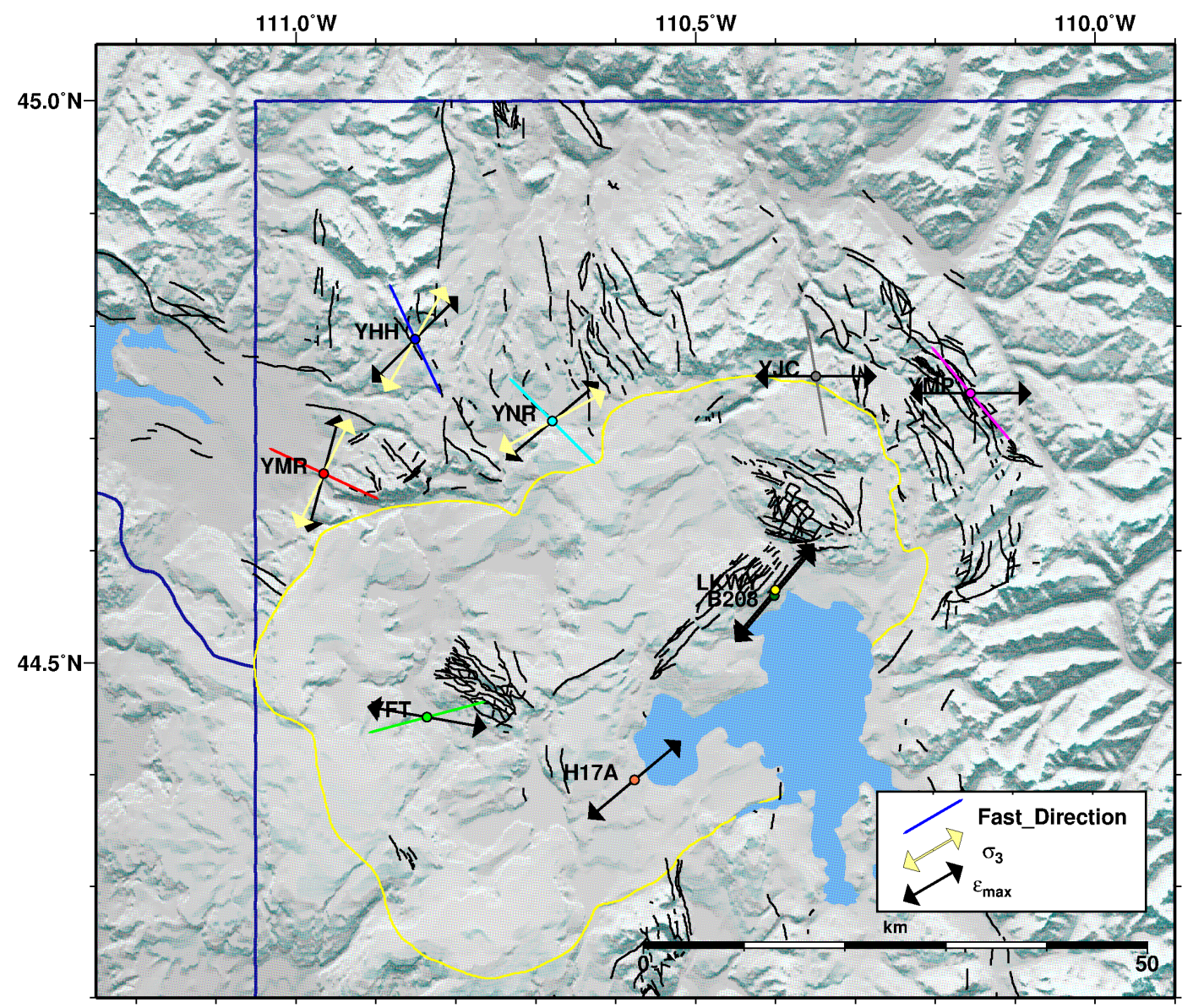

Figure 5.1 Map of preferred fast directions, local stress and strain. Maximum extensional strain $\left(\varepsilon_{\max }\right)$ directions are estimated from Smith et al. (2009) and minimum horizontal compression $\left(\sigma_{3}\right)$ directions are taken from Waite and Smith (2004) and plotted for comparison to the preferred fast directions. Stations B208, H17A, and LKWY have too few arrivals to discern a preferred fast direction. Stations YJC, YHH, YMR, and YNR exhibit $\varphi$ preferences nearly orthogonal to $\sigma_{3}$ and $\varepsilon_{\max }$, and stations YFT and YMP exhibit $\varphi$ preferences differing significantly from $\varepsilon_{\max }$. 
Table 5.1

Interpreted general fast directions vs. $\sigma_{3}$ and $\varepsilon_{\max }$. A non-value (--) indicates that a direction could not be determined due to limited data.

\begin{tabular}{|l|c|c|c|c|c|c|}
\hline \multirow{2}{*}{ Station } & \multicolumn{2}{|c|}{ Fast Direction } & \multicolumn{2}{c|}{$\begin{array}{c}\text { Minimum } \\
\text { Compressive Stress } \\
\left.\text { Direction( } \boldsymbol{\sigma}_{3}\right)\end{array}$} & \multicolumn{2}{c|}{$\begin{array}{c}\text { Maximum Horizontal } \\
\text { Strain Direction }\left(\boldsymbol{\varepsilon}_{\max }\right)\end{array}$} \\
\cline { 2 - 7 } & General & Estimated & General & Estimated & General & Estimated \\
\hline B208 & -- & -- & -- & -- & NE-SW & $40^{\circ}$ \\
\hline H17A & -- & -- & -- & -- & NE-SW & $50^{\circ}$ \\
\hline LKWY & -- & -- & -- & -- & NE-SW & $40^{\circ}$ \\
\hline YFT & ENE-WSW & $75^{\circ}$ & -- & -- & WNW-ESE & $100^{\circ}$ \\
\hline YHH & NNW-SSE & $155^{\circ}$ & NE-SW & $30^{\circ}$ & NE-SW & $45^{\circ}$ \\
\hline YJC & NNW-SSE & $170^{\circ}$ & -- & -- & E-W & $90^{\circ}$ \\
\hline YMP & NW-SE & $140^{\circ}$ & -- & -- & E-W & $90^{\circ}$ \\
\hline YMR & WNW-ESE & $115^{\circ}$ & NE-SW & $26^{\circ}$ & NNE-SSW & $15^{\circ}$ \\
\hline YNR & NW-SE & $135^{\circ}$ & NE-SW & $61^{\circ}$ & NE-SW & $50^{\circ}$ \\
\hline
\end{tabular}

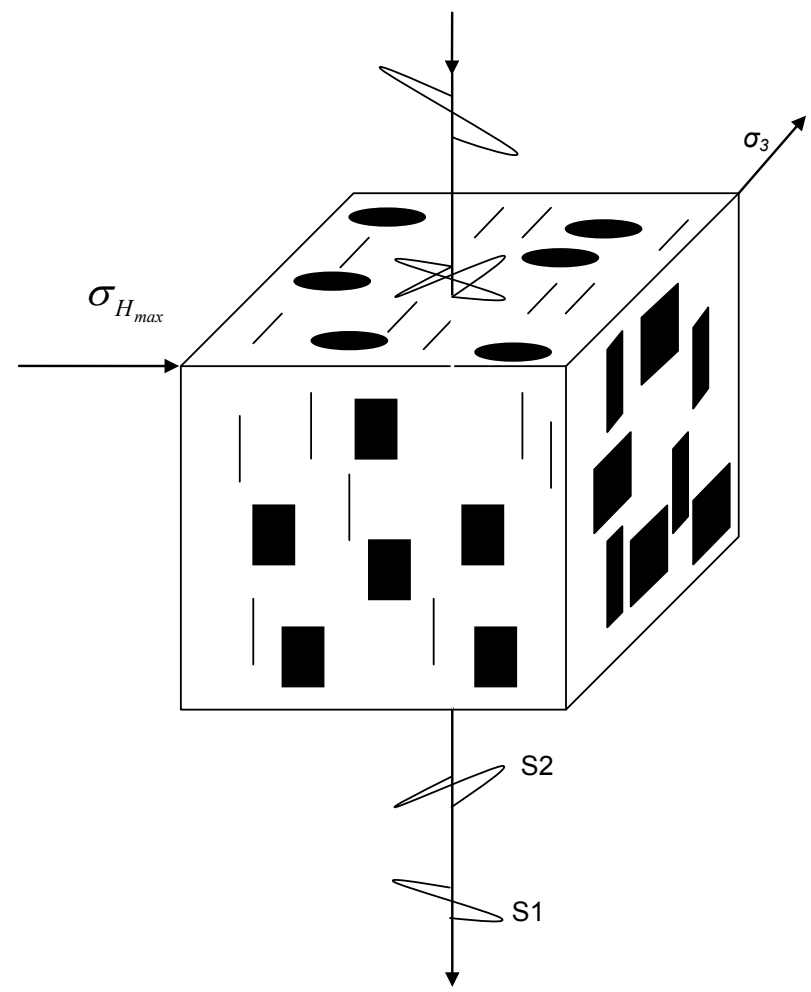

Figure 5.2 Schematic of crack induced anisotropy. A 3-D block of anisotropic medium is shown with cracks with faces oriented perpendicular to maximum horizontal compression close and cracks with faces oriented parallel to maximum horizontal compression close. Anisotropy is caused by the stress-induced alignment of fluid-filled microcracks. A shear wave entering from the top of the anisotropic medium will split into two orthogonally polarized waves: a fast wave (S1) and a slow wave (S2). The fast will be polarized parallel to the direction of maximum horizontal compression $\sigma_{H_{\max }}$. 
Pressurization of the hydrothermal-magmatic system within the caldera causes

deformation (e.g., Puskas et al. 2007) and increases compressive stresses along the rim of the caldera. As the direction of $\sigma_{\mathrm{H}_{\max }}$ rotates around the rim maintaining perpendicularity, so do the fast polarization directions of split shear waves arriving at the stations. This principle is portrayed well in the stations north and northwest of the caldera. Fast directions on station YMP to the NE of the caldera, rather than remaining perpendicular to $\sigma_{\mathrm{H}_{\max }}$ or the caldera rim, align sub-parallel to the general fault orientations near the station indicating that this station might be affected by fault-induced anisotropy rather than stress-aligned microcracks.

\subsection{Temporal Variation in Fast Directions}

Fast polarization directions have been used to study local crustal stress fields near volcanoes such as Mt. Ruapehu, New Zealand and Mt. Asama, Japan, and temporal variation has been suggested as a possible eruption forecasting tool (e.g., Miller and Savage 2001; Gerst and Savage 2004; Savage et al. 2010; Titzschkau et al. 2010). Changes in the local crustal stress field related to the pressurization of these volcanoes overprints the regional stress field, and it has been observed that these changes are detected in the fast polarization directions prior to and following eruptions. If the fast directions at Yellowstone are correlated to the local stress field, ideally we would be able to detect temporal changes in this stress field related to caldera deformation through changes in fast direction on our stations. We attempted to correlate temporal changes in fast direction with identified episodes of uplift and subsidence in the caldera. Figure 5.3 presents event fast directions as a function of time.

Stations B208, H17A, and YJC have too few events over too short of a period of time to conclude anything about change in fast direction. Likewise, station LKWY has too few events from which to draw conclusions. Stations YFT, YMP, and YNR do not show any considerable trends. On station YMR, we can clearly see the bimodal distribution in $\varphi$ 
values at orthogonal angles of $30^{\circ}$ and $120^{\circ}$, but no clear temporal variation. Station YHH, with 630 events over the 11 year time span, is the only station from which we might possibly be able to see a temporal variation. From 2000 - mid-2003, there are more events with $\varphi$ values less than $120^{\circ}$, than after mid-2003. This trend is slightly clearer in Figure 5.4 which presents fast direction as a function of a 60- and 180-day averages(circular mean) fit with quadratic functions. The general trend of fast directions on the 60-day average increases until 2009 at which point the trend flatlines. In the 180day average, we see an increase until mid-2006 and then a decreasing trend in fast direction. We can correlate these to the deformation history of the caldera as detailed by Chang et al. (2010). An unprecedented period of accelerated uplift began in 2004, which was followed from 2006-2008 by decreased acceleration, and a further decrease beginning in 2009, and ultimately a switch to subsidence in 2010. In the averaged fast direction data, we can see and increase and decrease in fast direction preference which indicates in the deformation behavior of the caldera. When looking at the fast directions in yearly increments as presented in Figure 5.5, we can see a general preference for fast directions less than $150^{\circ}$ prior to 2004 , and a greater than $150^{\circ}$ after 2004 .

We interpret this slight variation in fast polarization direction at station $\mathrm{YHH}$ as an indicator of change in the local crustal stress regime related to the switch from subsidence to uplift of the caldera in 2004 and subsequent decreasing uplift rates. During the accelerated uplift, compressive stress increased along the rim of the caldera leading to an increased number of closing microcracks with faces oriented subparallel to the caldera rim. The change in microcrack orientations led to the increase in fast polarization directions. The change, however, was not instantaneous as we can see increasing fast directions from 2000 to 2004 . This may indicate a rapidly decreasing subsidence rate leading up to the 2004 uplift. In 2006, while the caldera was still uplifting, the rate decreased and the pressure was slightly relieved along the caldera rim resulting in a decrease in fast polarization direction. 

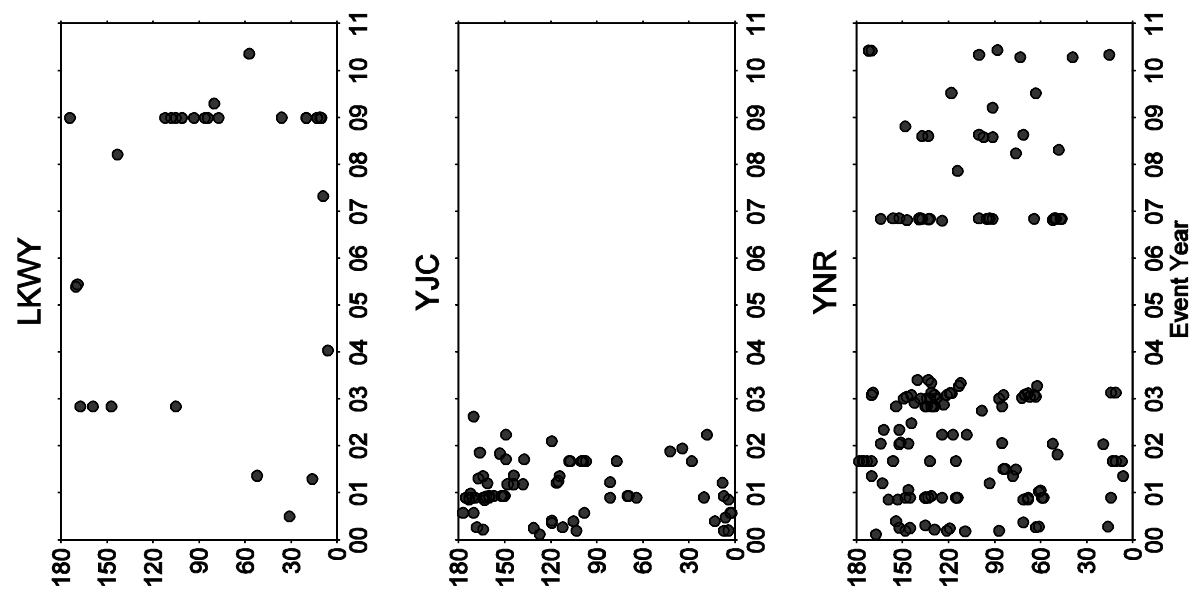

E 를

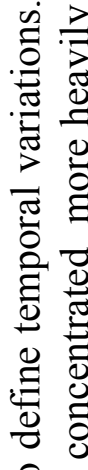

을

盯
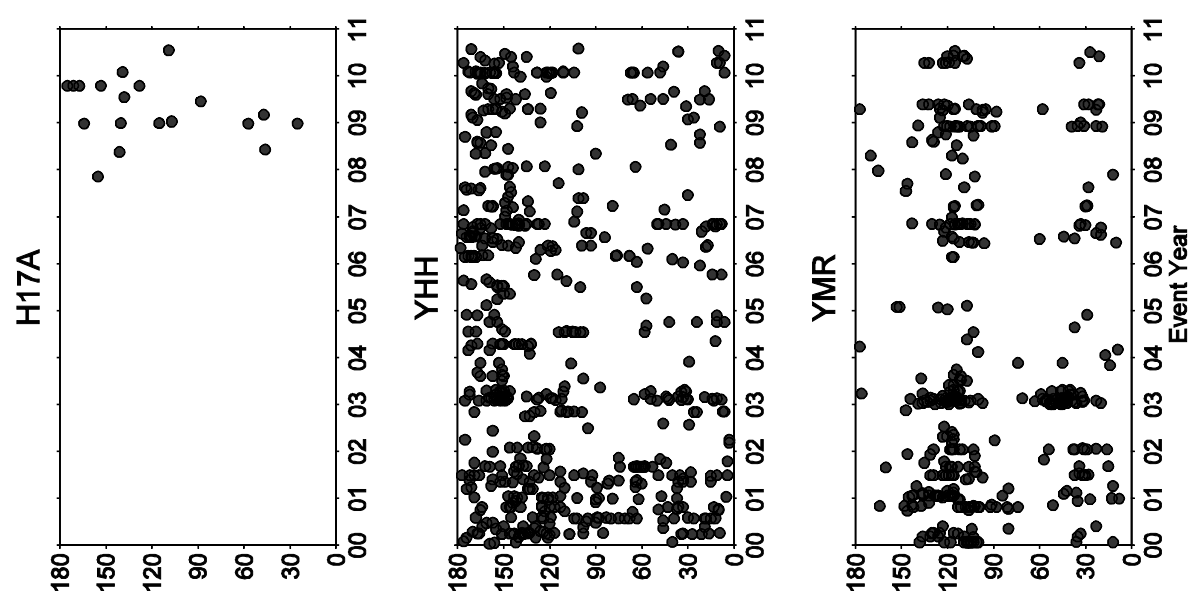

氞

이유

늉 른

을 $\frac{1}{3}$

䨔

3 吾

.유용

.

可

范 $\cong$

Ч 0

음으

․

夰
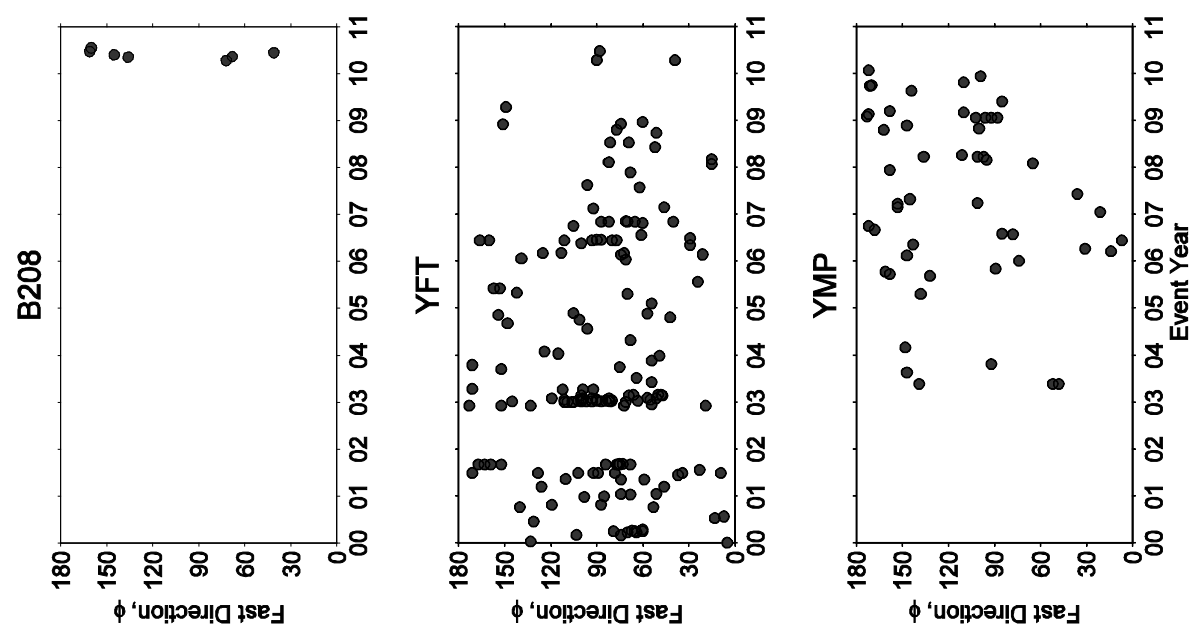

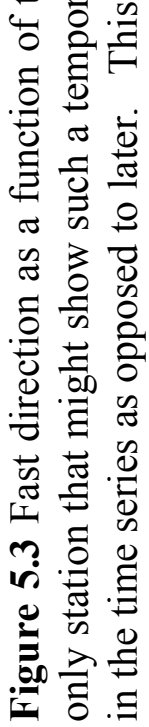



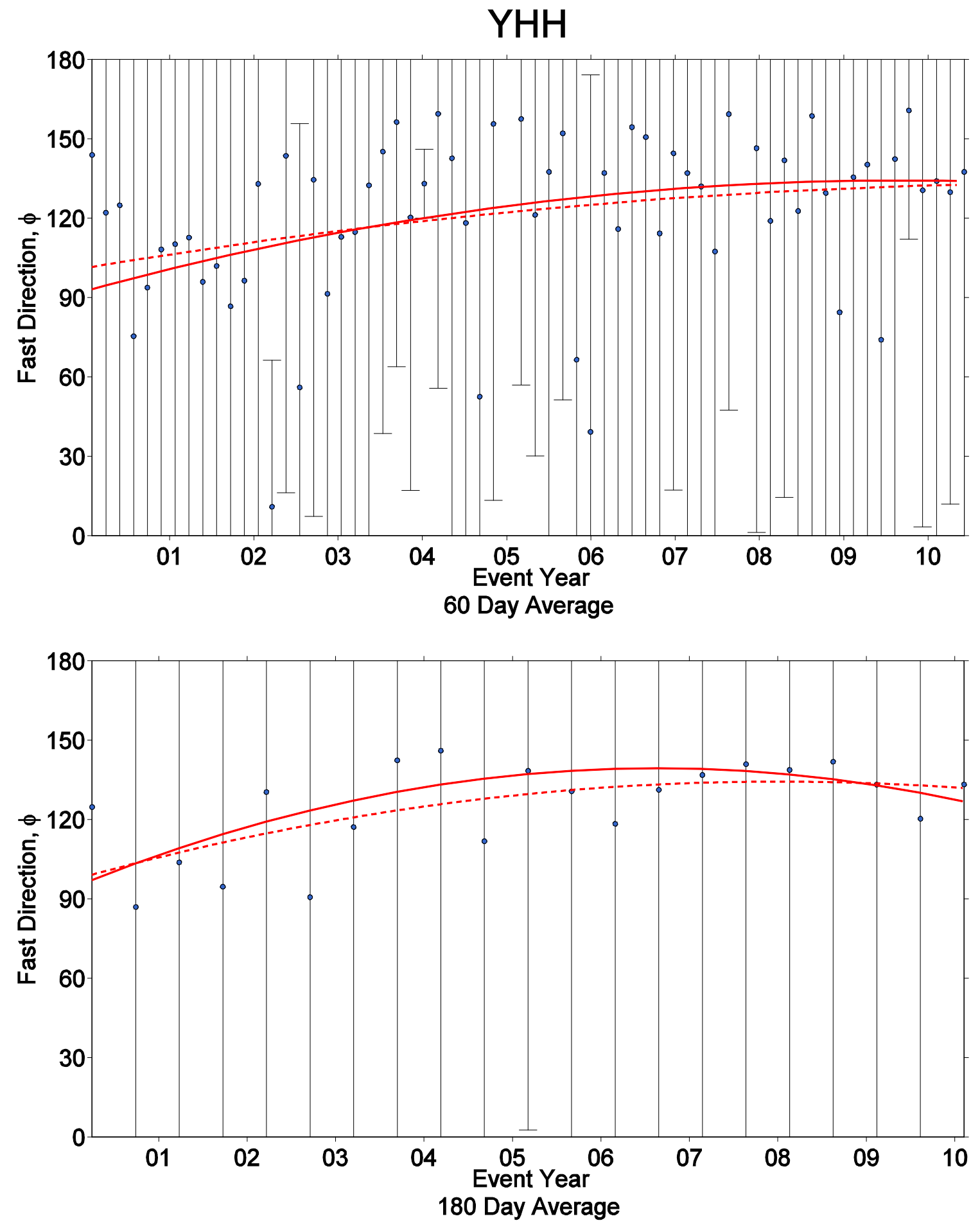

Figure 5.4 Fast direction vs. 60-day (top) and 180-day (bottom) average on station $\mathrm{YHH}$. Blue dots indicate average fast directions over the time period plotted with vertical error bars, many extending beyond the plot areas. The solid red line indicates a weighted least squared quadratic fit of the data, and the dashed red line indicates a non-weighted fit. 

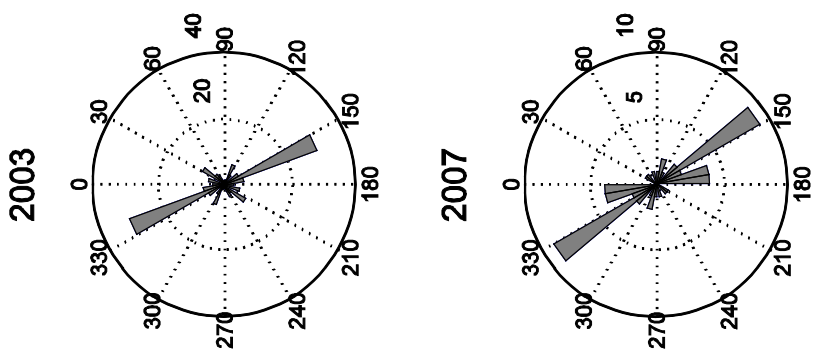

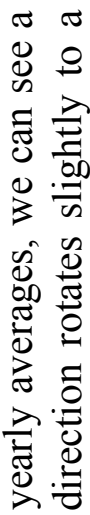

.윰
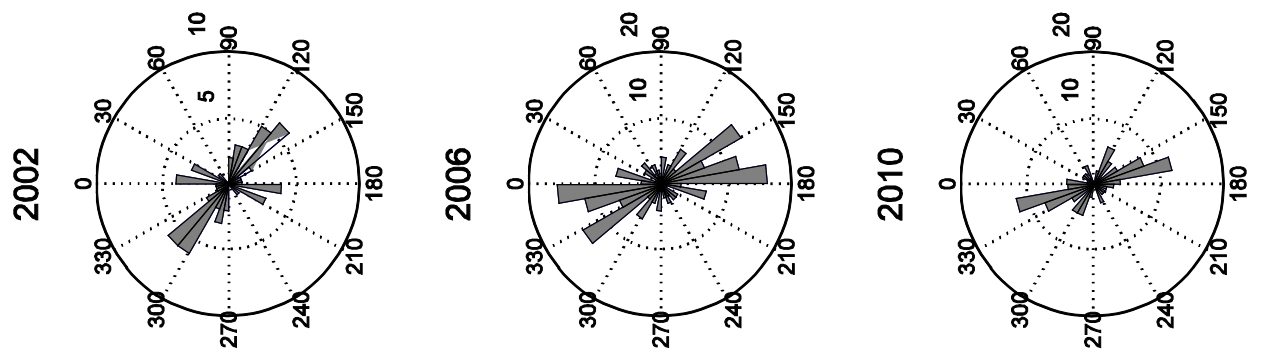

。

害名

离这

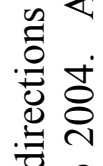

옹

을
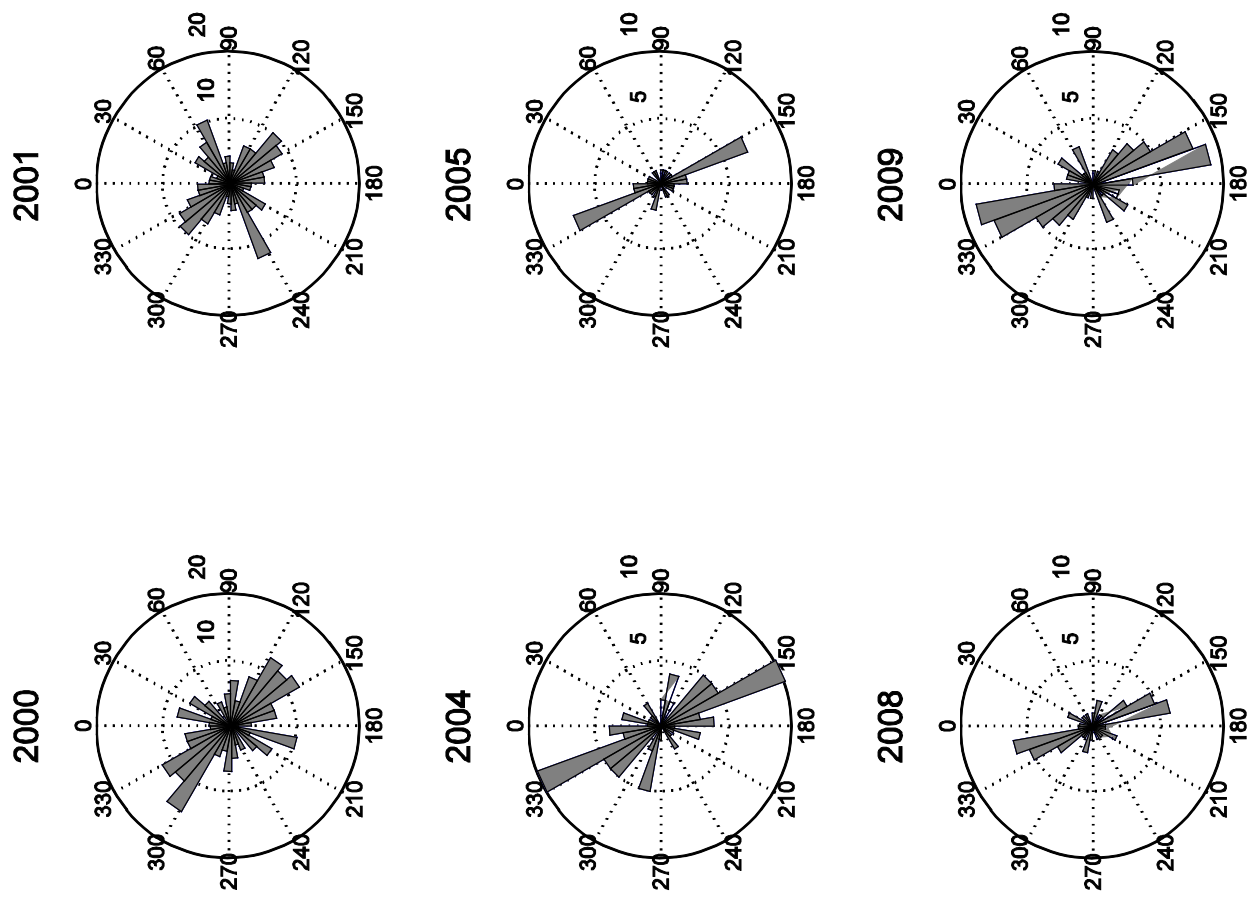

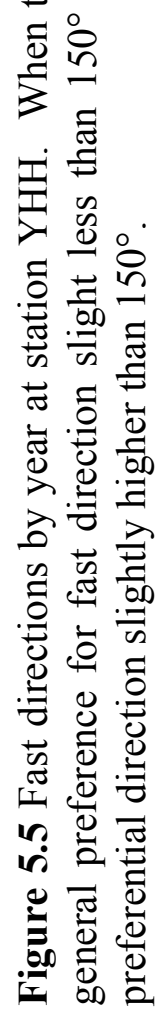


The trends on station YHH are certainly more discernable due to the a priori knowledge of the caldera deformation derived from GPS and InSar measurements. Noise in the data and scattered splitting estimates reduce the definition of these trends on station $\mathrm{YHH}$ as well as the others. Gerst and Savage (2004) used shear-wave splitting to monitor microcrack orientation and temporal variations at Mt. Ruapehu, New Zealand, and they were able to detect a change in crustal anisotropy orientation by up to $80^{\circ}$ related to changes in pressure of the magmatic system. Despite similar periods of intense deformation at Yellowstone (e.g., the accelerated uplift episode beginning in 2004), we don't see strong correlative changes in shear-wave splitting estimates.

\subsection{Fast Directions vs. Back Azimuth}

Supposing that the local stress field deviation at Yellowstone is influenced by the deformation of the caldera, we might assume that the seismic anisotropy of earthquakes originating beyond the rim to station line will be less affected by the stress-oriented microcracks than those earthquakes originating within the caldera or between the caldera rim and station. Polarization directions are primarily affected by anisotropy within a few wavelengths of the station (Nistala and McMechan 2005). Assuming an average shearwave velocity of $1.7 \mathrm{~km} / \mathrm{s}$, and shear-wave frequency of $4.5 \mathrm{~Hz}$, one wavelength is approximately $400 \mathrm{~m}$. If we presume that $\varphi$ is derived from the last four wavelengths $(1.6 \mathrm{~km})$ of the shear-wave raypath, we are still well within the local crustal stress field overprint of the caldera. We examined fast directions as a function of back azimuth to determine if the geographically rotating stress field can be detected with shear-wave splitting. The results are presented in Figure 5.6.

On station, YHH, we can't see any trends in the dataset as a whole, but with a $10^{\circ}$ average of the back azimuth (Fig. 5.7), we can see an increase in $\varphi$ with back azimuth. On station YMR, in the whole dataset we can a decrease in $\varphi$ with back azimuth on both the primary fast direction $120^{\circ}$ and the orthogonal angle $30^{\circ}$. However, on the $10^{\circ}$ average, we see an increase of $\varphi$ with the back azimuth. This disparity on station YMR is 
likely due to the bimodality of fast direction preferences at orthogonal angles and a greater number of events having $120^{\circ}$ fast direction at larger back azimuths. Assuming that stations YHH and YMR do show back-azimuthal dependence, and these trends are not simply artifacts of scattered data, the data corroborate the rotating crustal stress directions NW of the caldera.

\subsection{Fast Directions vs. Frequency Band}

We filtered the data prior to splitting parameter estimation in order to progressively remove noise and focus in on the shear-wave frequency $(4-5 \mathrm{~Hz})$ as well as study stability of estimates over different frequency bands. In order to study these effects, we performed the automated cluster analysis on estimates over single frequency bands and the results are presented in Figures 5.8-5.10. In these data, the minimum number of data points required for optimum cluster consideration was removed to accommodate the reduction in data points and cycle skipping has not been corrected.

It is interesting to note that while stations B208 and H17A did not exhibit preferential fast directions when all frequency bands were combine prior to cycle skipping elimination (Fig. 4.2), they do exhibit preferential fast directions in a few of the individual frequency bands. Station B208 has a NW-SE preference on Bands 2 and 3 and rotates to a NE-SW preference on Bands 4 and 5. Station H17A shows a NE-SW preference on Bands 1 and 2, a NNW-SSE preference on Band 3, and diminished preference on Bands 4 and 5. The combination of these differing fast directions likely results in the lack of fast direction preference in the whole dataset. Station LKWY has a general N-S fast direction preference on Bands 3-5 that correspond to the preference seen in the whole data set.

Stations YFT, YHH, and YJC each show comparable fast direction preferences on all five frequency bands and station YHH and YJC fast directions match those presented in the data as a whole. YFT however, does not exhibit the orthogonal $150^{\circ}$ preference that 

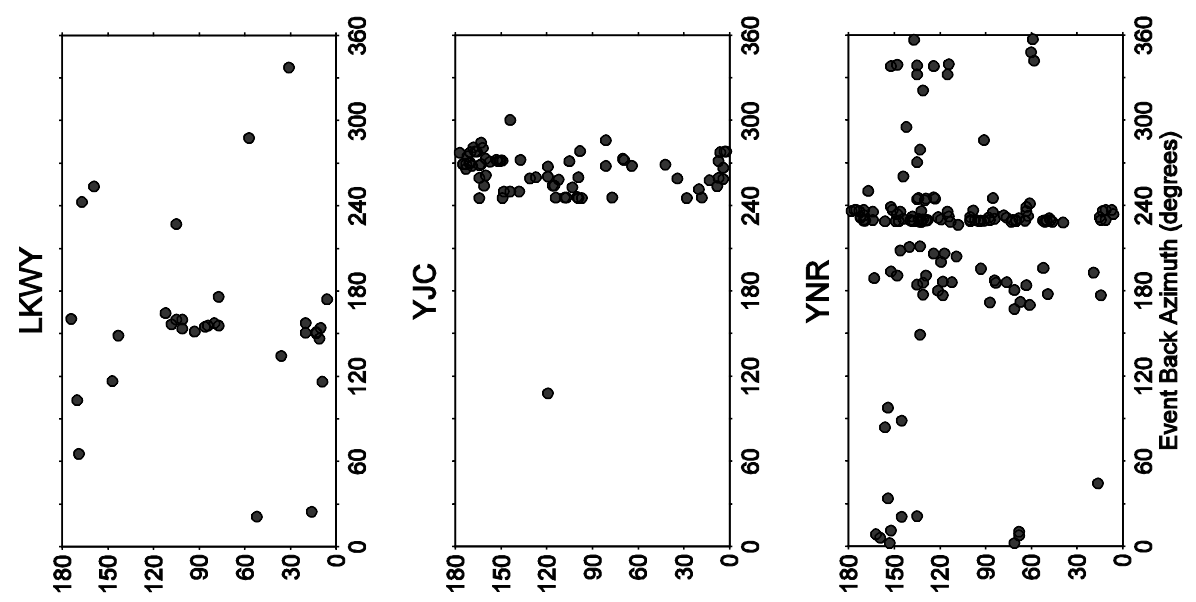

\&

हี

३:

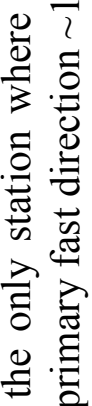

过

馬
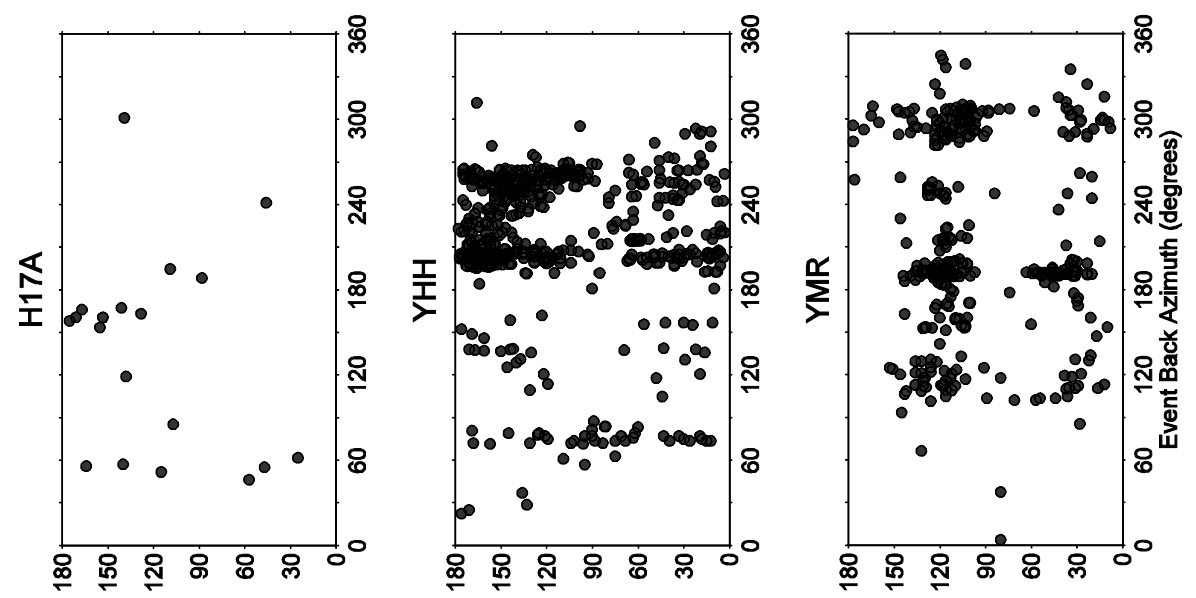

워

릭

응 일

झ焉

३ 0

욜

.

처

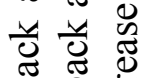

อ ป

世宁

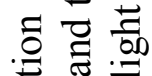
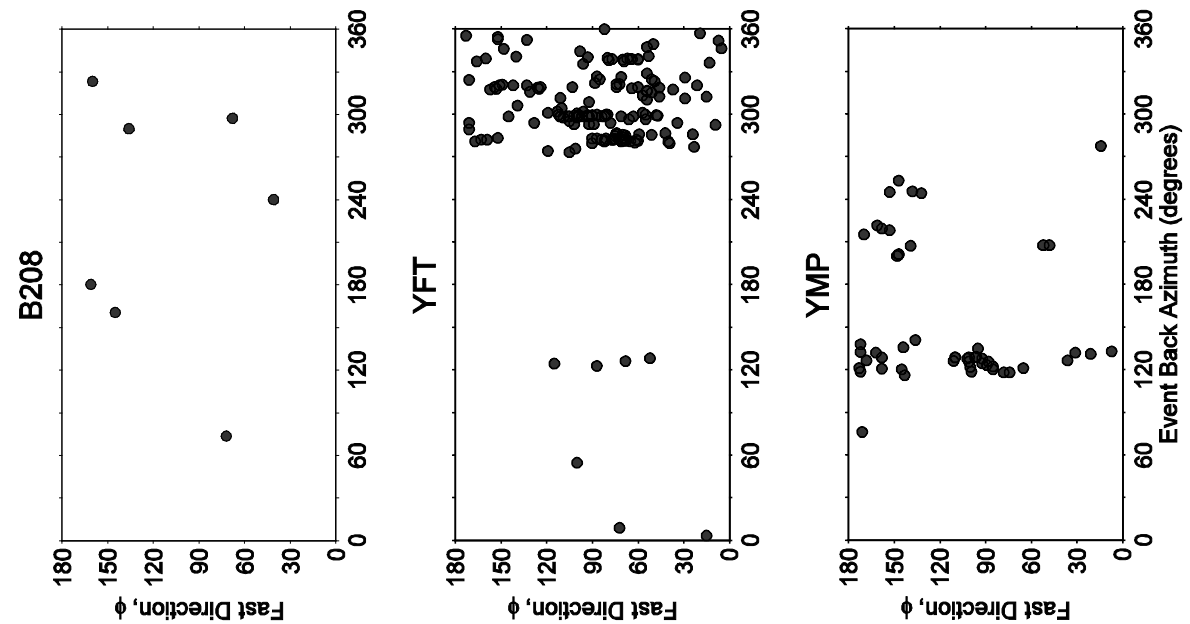

U

豆

๙ ల ల

ส

范芯

苍

च

范令

工

ம் 증

츨

일 

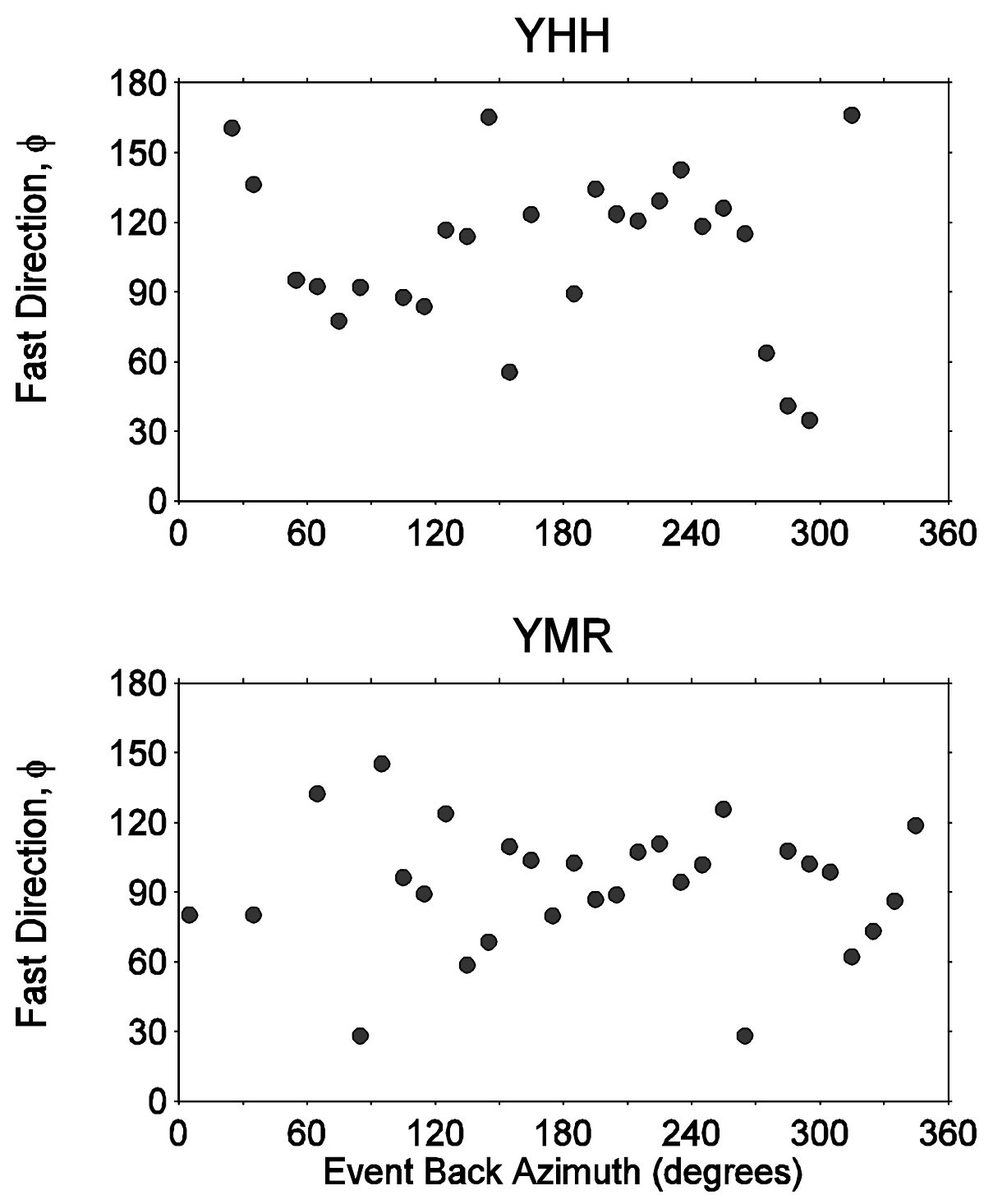

Figure 5.7 Fast direction as a function of a $10^{\circ}$ back azimuth average. The increasing trend of phi with back azimuth on YHH is clearer whereas the decreasing trend that was present on YMR in the entire dataset is replaced by an increasing trend in the average. This disparity is likely due to the bimodal nature of fast directions on $\mathrm{YMR}$ at $30^{\circ}$ and $120^{\circ}$. 
dominates the whole data set and was attributed to cycle skipping. It is possible that within individual frequency bands, relatively few estimates are affected by cycle skipping, and therefore the automated cluster analysis chooses appropriate optimum measurements. When all five frequency bands are clustered together, however, the combination of estimates affected by cycle skipping could dominate those that are not and this is reflected in the optimum measurements.

Station YMP exhibits a general N-S fast direction preference on all five frequency bands, but in a much less definitive manner than is present in the whole dataset. Station YMR is still plagued by the bipolar nature of fast direction preferences at orthogonal angles, and on Band 3, the minor fast direction dominates. Station YNR has a general NW-SE fast direction that is present in the whole dataset on all but Band 3 .

It seems that station B208, H17A, LKWY are most affected by the different frequency bands. However, we must recognize that these stations have relatively few events compared to the remaining stations and perhaps there are too few events to determine a definitive fast direction preference. We find that there is a general fast direction stability over the five frequency bands on the remaining stations which indicates that splitting estimates are more sensitive to the selection of the beginning and ending of the time analysis window than the filtered frequency bands, and thus it is not necessary to completely isolate the shear wave frequency in analysis.

\subsection{Calculating Anisotropy}

With splitting parameters determined we can characterize the anisotropic medium through which the shear-waves are travelling. Delay times can be used to calculate the percentage of crustal anisotropy (e.g., Savage 1999; Savage et al. 2010) as well as microcrack density (e.g., Hudson 1981; Savage et al. 2010). Delay time can be related to anisotropy through the following formulas (Savage 1999): 

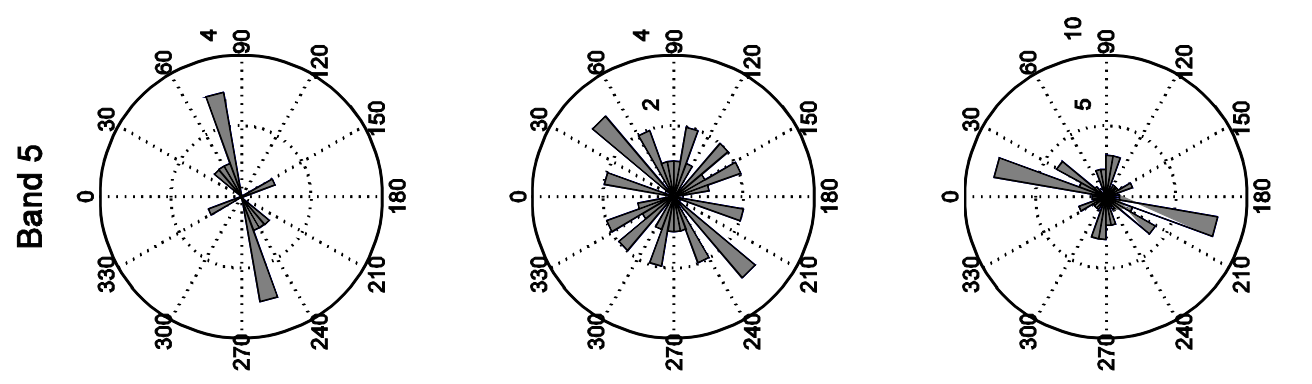

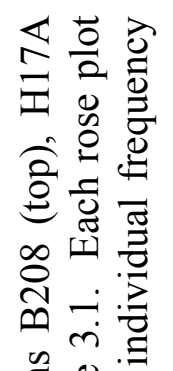
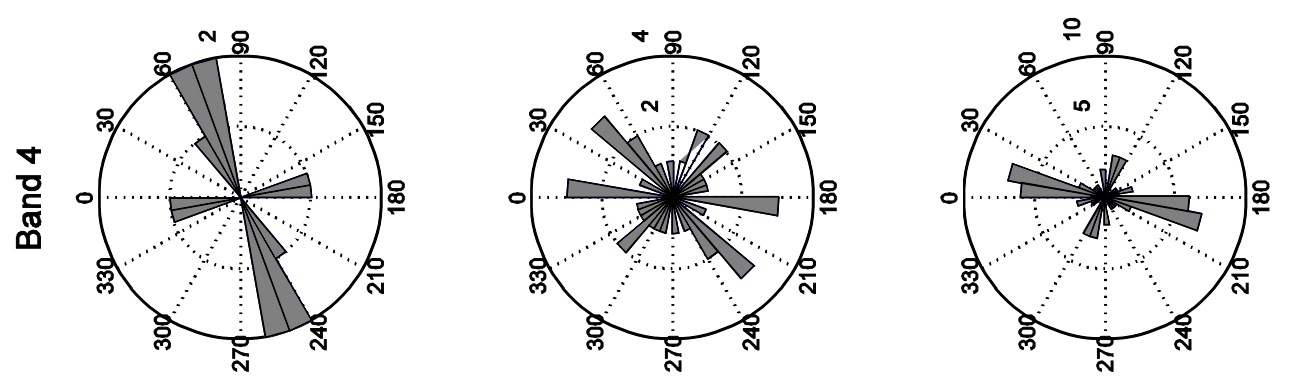

号啇

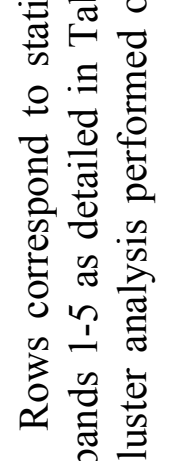
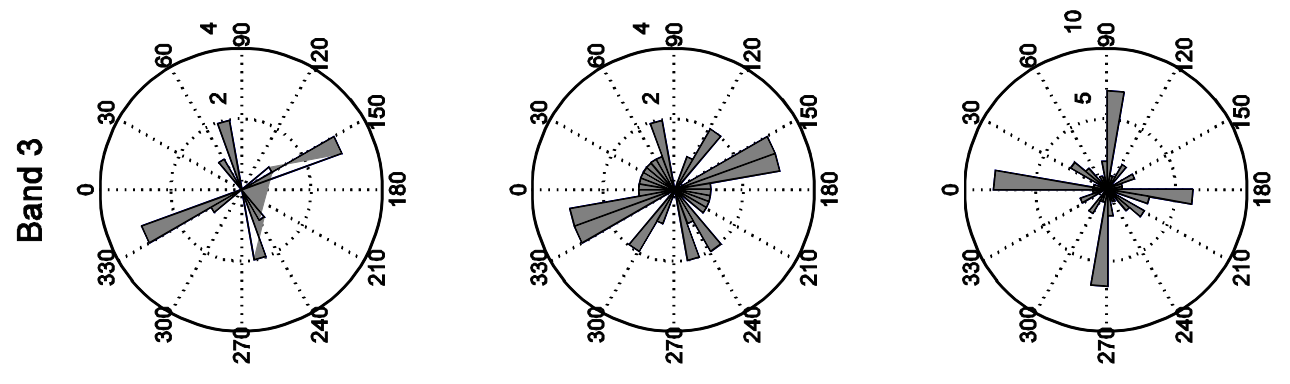

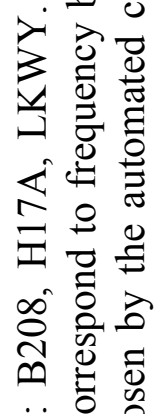
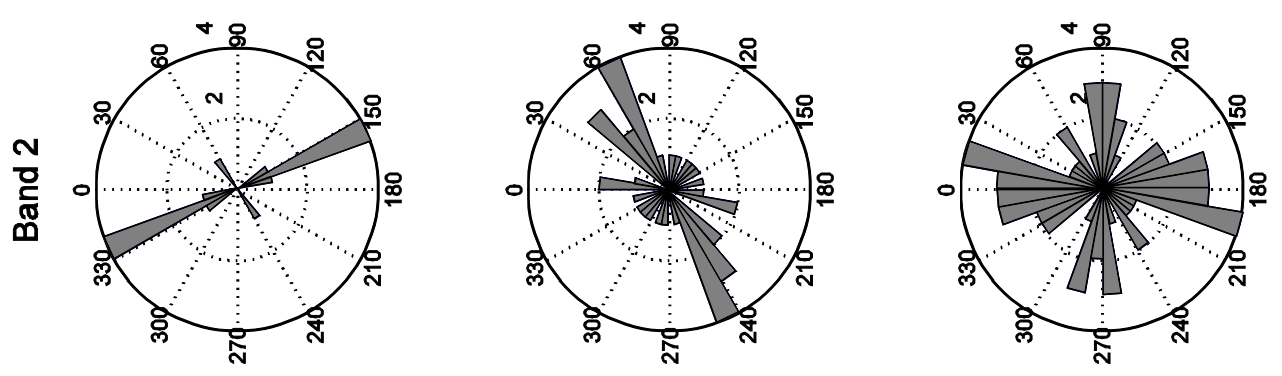

تृ 0 ठ

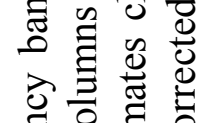

응

可矛

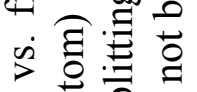

व

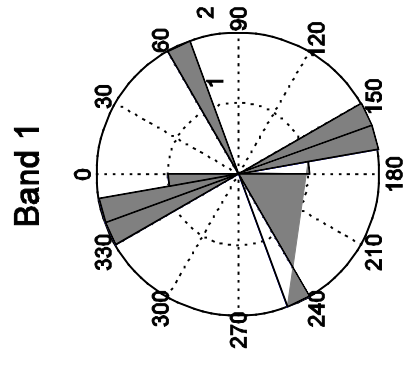

8028

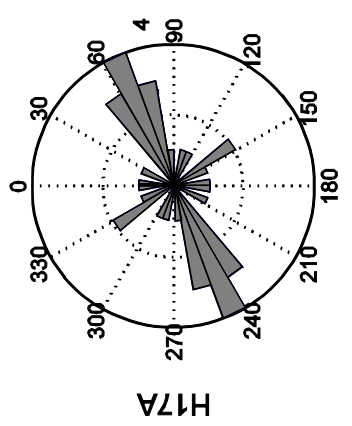

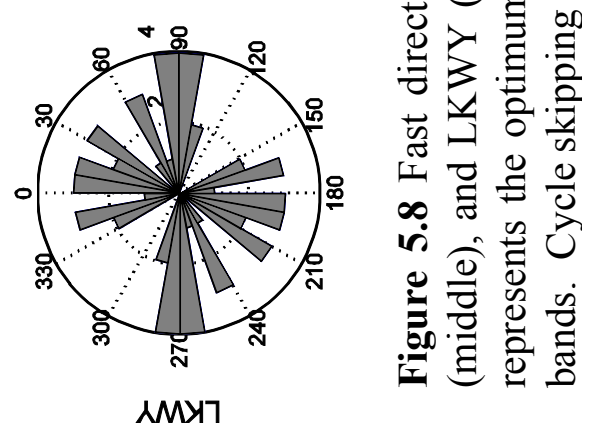



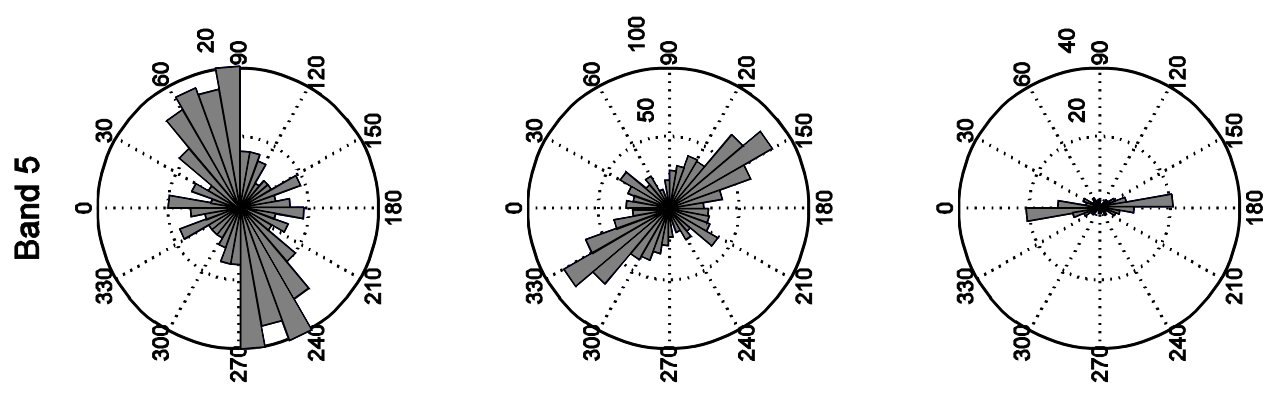

ì
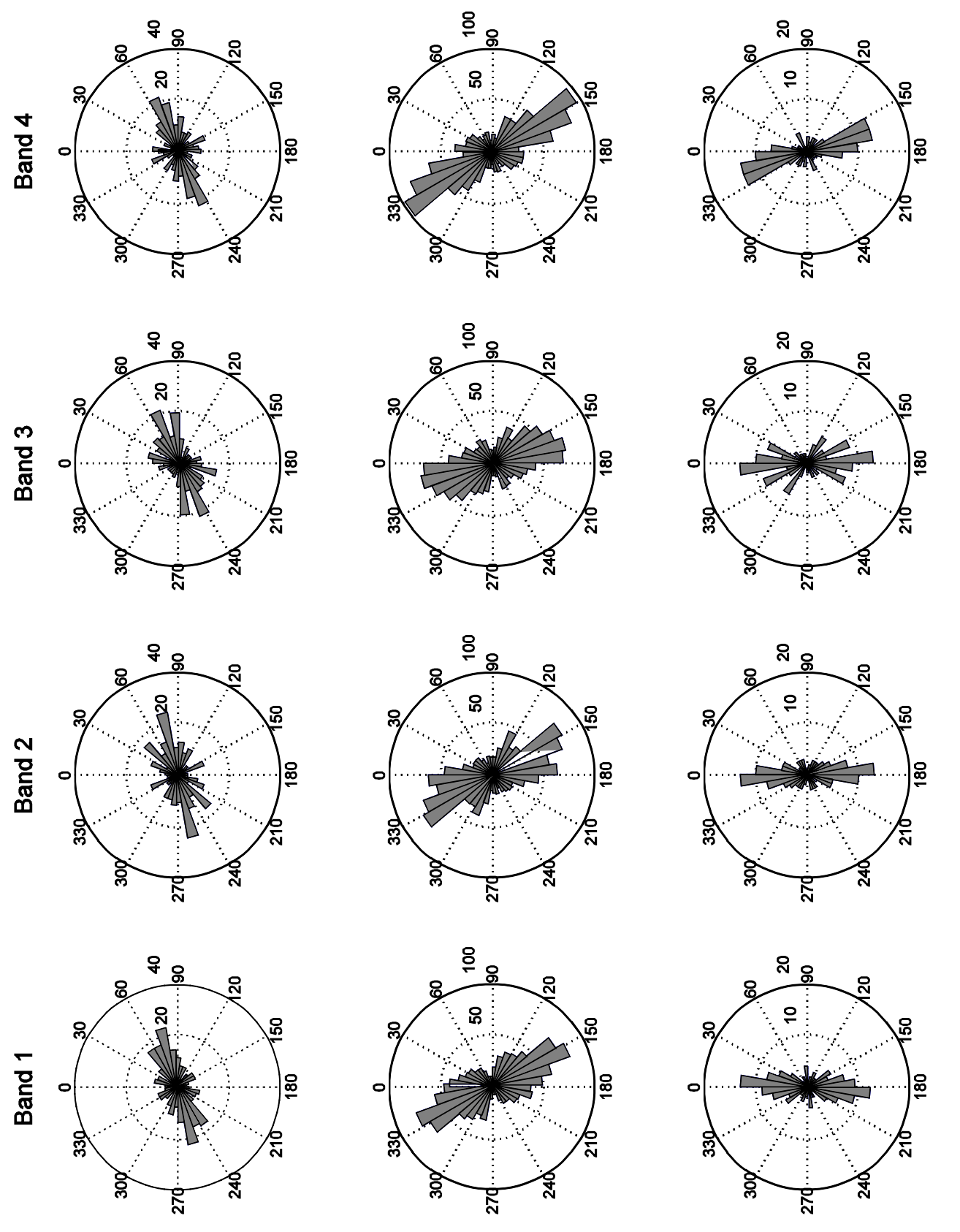

넛ㅅ

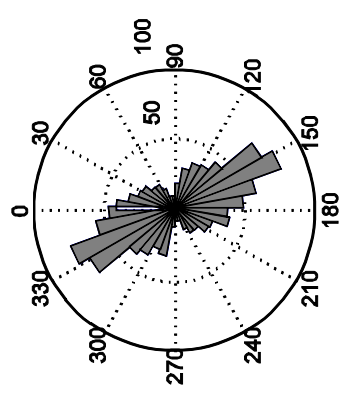

$\mathrm{HH}$ 人
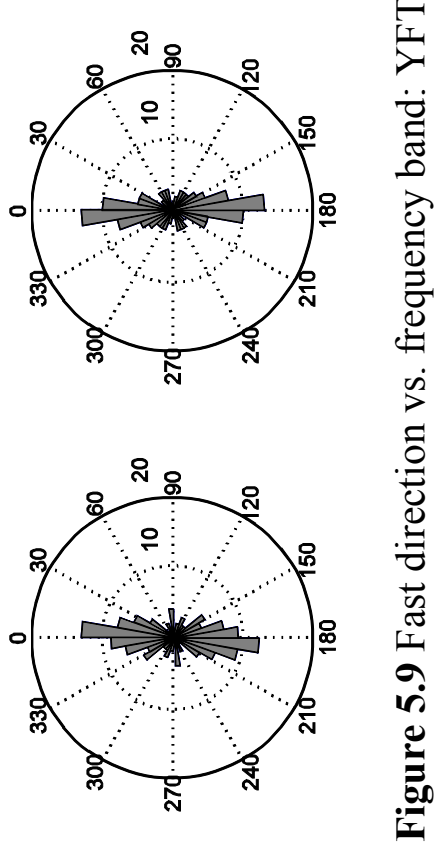

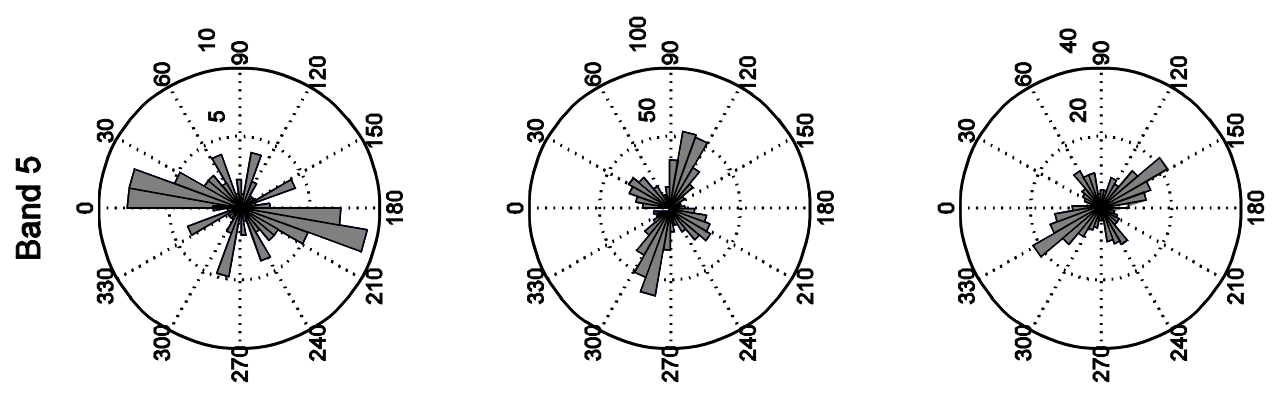

ำ
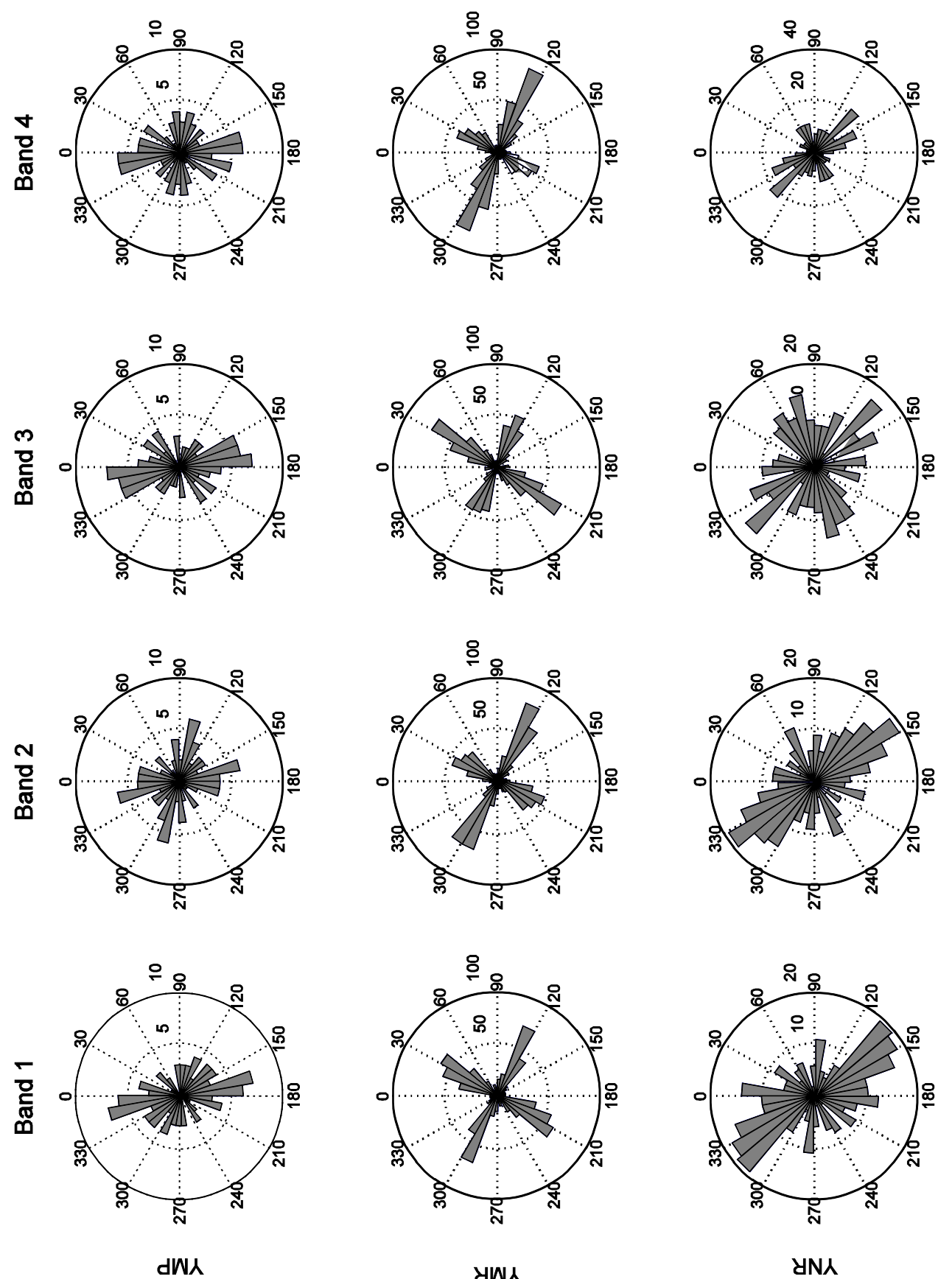


$$
\begin{aligned}
& \delta t=\frac{L\left(v_{S 1}-v_{S 2}\right)}{v_{S 1} v_{S 2}}, \\
& P_{A}=\frac{200\left(v_{S 1}-v_{S 2}\right)}{v_{S 1}+v_{S 2}},
\end{aligned}
$$

where $\delta t$ represents the average delay time, $L$ is the path length over which splitting occurs, $v_{S 1}$ and $v_{S 2}$ are the velocities and the fast and slow shear waves, and $P_{A}$ is the anisotropy. Crack density $\rho$ is related to delay time by the following formula (Hudson 1981):

$$
\rho=\frac{7 \delta t v_{S}}{8 L}
$$

where $v_{S}$ is the average shear wave velocity.

Average delay times weight by errors were calculated for each of the stations as listed in Table 5.2 as well as a delay time weighted averaged over all stations. We assume a path length $L=1 \mathrm{~km}$, the critical depth determined by Crampin and Chastin (2003) below which the vertical stresses dominate the horizontal stresses. We also studied the dependence of delay time on event to station distance and found no correlation. Therefore, we assume that the path length through the anisotropic medium is constrained to less than the shortest event to station distance at approximately $1 \mathrm{~km}$. We assume an average upper crust shear wave velocity of $1.7 \mathrm{~km} / \mathrm{s}$ (Husen et al. 2004). With these values we calculated the percent anisotropy to be $7-23 \%$ with an average of $17 \%$, and crack densities to be $0.055-0.17$ with an average of 0.15 .

We again conclude that stations B208 and H17A have too few events and we cannot confidently calculate an average delay time, therefore percent anisotropy and crack densities will be disregarded. Further supporting this disregard of values calculated for B208 is the $12 \%$ difference in anisotropy between stations B208 and LKWY which are only separated by a distance of $1.1 \mathrm{~km}$. Crustal anisotropies on the remaining seven 
stations between 17 and 23\% are on par with the crustal anisotropy calculated at Long Valley Caldera, California (Savage et al. 1990; Shih et al. 1990; Crampin 1994) where high crustal anisotropy values were found in a volcanic rock fracture zone with high heat flow, similar to the geologic conditions present at Yellowstone.

According to Crampin (1994), crustal anisotropies above 10\%, and corresponding crack densities above 0.1 correlate to heavily fractured rocks which are constrained to the upper $1 \mathrm{~km}$. Crack densities greater than 0.1 only exist when fluid-pore pressure is high enough to sustain open cracks. It is reasonable to assume that the large hydrothermal system at Yellowstone resultant from circulating hot water in fracture systems heated by shallow crystallizing magma (Fournier 1989) is sufficient to prop open cracks and cause large anisotropies.

The largest anisotropies are found northwest of the caldera at stations YHH and YMR correlating to the region where the majority of Yellowstone seismicity is located. Husen and Smith (2004) postulate that the intense seismicity in the region might be attributable to the increased Coulomb failure stress on the late Quaternary faults due to the 1959 Hebgen Lake earthquake, which decreases east of the Norris Geyser Basin. High anisotropy could possibly be related to the increased failure stress and the relatively low anisotropy at station YNR could be related to the eastward decrease. Anisotropies values match on close proximity stations YJC and YMP northeast of the caldera, and a slightly lesser anisotropy value is found on station YFT within the caldera. 
Table 5.2 Calculated percent anisotropies and crack densities.

\begin{tabular}{|l|c|c|c|}
\hline Station & $\begin{array}{c}\text { Average } \\
\text { Delay Time } \\
\boldsymbol{\delta} \boldsymbol{t}(\mathbf{s})\end{array}$ & $\begin{array}{c}\text { Percent } \\
\text { Anisotropy } \\
\boldsymbol{P}_{\boldsymbol{A}}\end{array}$ & $\begin{array}{c}\text { Crack } \\
\text { Density } \\
\boldsymbol{\rho}\end{array}$ \\
\hline B208 & 0.037 & 7.3 & 0.055 \\
\hline H17A & 0.075 & 15.0 & 0.11 \\
\hline LKWY & 0.097 & 19.4 & 0.14 \\
\hline YFT & 0.088 & 17.5 & 0.13 \\
\hline YHH & 0.109 & 21.8 & 0.16 \\
\hline YJC & 0.094 & 18.8 & 0.14 \\
\hline YMP & 0.093 & 18.7 & 0.14 \\
\hline YMR & 0.114 & 22.8 & 0.17 \\
\hline YNR & 0.084 & 16.8 & 0.12 \\
\hline All Stations & 0.103 & 17.4 & 0.15 \\
\hline
\end{tabular}




\section{Conclusions}

By automating the calculation of shear-wave splitting parameters, we eliminate user bias and allow for processing of much more data. The analysis window can be sensitive to small changes, but cycling through a series of start and end times defines a set of windows over which splitting estimates are stable. We found that splitting parameter estimations were stable over different frequency bands eliminating the need to isolate the shear-wave frequency in analysis. Cluster analysis can be an excellent technique for unsupervised choice of optimum splitting parameters. Unfortunately, our measurements are plagued by effects of cycle skipping which can lead to picking of ineffective splitting parameters. Our method for solving the cycle skipping problem does require a priori knowledge of the event data and multiple user inputs, resulting in a semi-automated method for parameter determination.

Our method was effective in helping to determine the fast polarization directions at 6 of the 9 stations studied. Stations B208, H17A and LKWY had too few pickable events and thus reliable results could not be gleaned from the data. Fast polarization directions on the remaining stations did align normal to or at a significant angle to the direction of maximum extensional strain/minimum horizontal compressive stress which differ from the regional stress field, proving that shear-wave splitting can be a useful indicator of crustal stresses.

Temporal variations in the fast polarization direction were detected only on station $\mathrm{YHH}$ because of the large amount of data on this station. These variations correlate with GPS identified periods of caldera subsidence and uplift and may also reflect changes in the rates of subsidence and uplift. Scatter in the data and relatively short periods of deployment prevented the detection of temporal variation on the other stations. We found that in areas of complex crustal stress fields, such as northwest of the caldera, splitting parameters may reflect back azimuthal dependence. 
From the average delay times, we calculated an anisotropy $\sim 17-23 \%$ corresponding to a crack density of $0.12-0.17$ which is on par with values calculated for a similar geological region in Long Valley Caldera, California. 


\section{Reference List}

Blackwell DD. 1969. Heat-flow determinations in the northwestern United States. Journal of Geophysical Research 74(4):992-1007.

Booth DC, Crampin S. 1985. Shear-wave polarizations on a curved wavefront at an isotropic free surface. Geophysical Journal of the Royal Astronomical Society 83(1):31-45.

Caliński T, Harabasz J. 1974. A dendrite method for cluster analysis. Communications in Statistics 3(1):1 - 27.

Chang WL, Smith RB, Wicks C, Farrell JM, Puskas CM. 2007. Accelerated uplift and magmatic intrusion of the Yellowstone caldera, 2004 to 2006. Science 318(5852):952-956.

Chang WL, Smith RB, Farrell J, Puskas CM. 2010. An extraordinary episode of Yellowstone caldera uplift, 2004-2010, from GPS and InSAR observations. Geophysical Research Letters 37(23):L23302.

Christiansen RL. 2001. The Quaternary and Pliocene Yellowstone Plateau volcanic field of Wyoming, Idaho, and Montana. U. S. Geological Survey Professional Paper:G1-G145.

Christiansen RL, Lowenstern JB, Smith RB, Heasler H, Morgan LA, Nathenson M, Mastin LG, Muffler LJP, Robinson JE. 2007. Preliminary assessment of volcanic and hydrothermal hazards in Yellowstone National Park and vicinity. Open-File Report - U. S. Geological Survey.

Crampin S. 1981. A review of wave motion in anisotropic and cracked elastic-media. Wave Motion 3(4):343-391.

Crampin S, Booth DC. 1985. Shear-wave polarizations near the North Anatolian Fault; II, Interpretation in terms of crack-induced anisotropy. Geophysical Journal of the Royal Astronomical Society 83(1):75-92.

Crampin S. 1994. The fracture criticality of crustal rocks. Geophysical Journal International 118(2):428-438.

Crampin S, Chastin S. 2003. A review of shear wave splitting in the crack-critical crust. Geophysical Journal International 155(1)221-240.

Doser DI. 1985. Source parameters and faulting processes of the 1959 Hebgen Lake, Montana, earthquake sequence. Journal of Geophysical Research 90(B6):4537-4555.

Duda RO, Hart PE. 1973. Pattern Classification and Scene Analysis. New York: Wiley.

Farrell J, Husen S, Smith RB. 2009. Earthquake swarm and b-value characterization of the Yellowstone volcano-tectonic system. Journal of Volcanology and Geothermal Research 188(1-3):260-276. 
Farrell J, Smith RB, Puskas CM, Taira T, Chang WL. 2010. Dynamics and rapid migration of the energetic 2008-2009 Yellowstone Lake earthquake swarm. Geophysical Research Letters 37(19):L19305.

Fournier RO. 1989. Geochemistry and dynamics of the Yellowstone National Park hydrothermal system. Annual Review of Earth and Planetary Sciences 17:13-53.

Gerst A, Savage MK. 2004. Seismic anisotropy beneath Ruapehu Volcano: A possible eruption forecasting tool. Science (Washington) 306(5701):1543-1547.

Gnanadesikan R, Kettenring JR, Tsao SL. 1995. Weighting and selection of variables. Journal of Classification 12:113-136.

Gripp A, Gordon R. 2002. Young tracks of hotspots and current plate velocities. Geophysical Journal International 150:321-361.

Haslinger F, Kissling E. 2001. Investigating effects of 3-D ray tracing methods in local earthquake tomography. Physics of The Earth and Planetary Interiors 123(2-4):103-114.

Hudson J. 1981. Wave speeds and attenuation of elastic waves in material containing cracks. Geophysical Journal of the Royal Astronomical Society 64(1):133-150.

Husen S, Smith RB. 2004. Probabilistic earthquake resolution in three-dimensional velocity models for the Yellowstone National Park region, Wyoming. Bulletin of the Seismological Society of America 94(3):880-896.

Husen S, Smith RB, Waite GP. 2004. Evidence for gas and magmatic sources beneath the Yellowstone volcanic field from seismic tomographic imaging. Journal of Volcanology and Geothermal Research 131(3-4):397-410.

Levin V, Menke W, Park J. 1999. Shear wave splitting in the Appalachians and the Urals; a case for multilayered anisotropy. Journal of Geophysical Research 104(17):17975-17994.

Long MD, van der Hilst RD. 2005. Upper mantle anisotropy beneath Japan from shear wave splitting. Physics of The Earth and Planetary Interiors 151(3-4):206-222.

Lou M, Rial JA. 1997. Characterization of geothermal reservoir crack patterns using shear-wave splitting. Geophysics 62(2):487-494.

Matcham I, Savage MK, Gledhill KR. 2000. Distribution of seismic anisotropy in the subduction zone beneath the Wellington region, New Zealand. Geophysical Journal International 140(1):1-10.

Meertens CM, Smith RB. 1991. Crustal deformation of the Yellowstone Caldera from first GPS measurements; 1987-1989. Geophysical Research Letters 18(9):1763-1766.

Miller V, Savage M. 2001. Changes in seismic anisotropy after volcanic eruptions: Evidence from Mount Ruapehu. Science 293(5538):2231-2233. 
Milligan GW, Cooper MC. 1985. An examination of procedures for determining the number of clusters in a data set. Psychometrika 50(2):159-179.

Milligan GW, Cooper MC. 1988. A study of standardisation of variables in cluster analysis. Journal of Classification 5:181-204.

Murdock JN. 1978. Travel time and spectrum stability in the two years preceding the Yellowstone, Wyoming, earthquake of June 30, 1975. Journal of Geophysical Research 83(B4):1713-1717.

Nistala S, McMechan GA. 2005. 3D modeling of fracture-induced shear-wave splitting in the Southern California basin. Bulletin of the Seismological Society of America 95(3):10901100 .

Peacock S, Crampin S, Booth D, Fletcher J. 1988. Shear wave splitting in the Anza seismic gap, Southern California; temporal variations as possible precursors. Journal of Geophysical Research 93(B4):3339-3356.

Perkins ME, Nash BP. 2002. Explosive silicic volcanism of the Yellowstone hotspot: The ash fall tuff record. Bulletin of the Geological Society of America 114(3):367-381.

Pitt AM. 1987. Catalog of earthquakes in the Yellowstone National Park -- Hebgen Lake region, Wyoming, Montana, Idaho, for the years 1973 to 1981. Open-File Report - U. S. Geological Survey.

Puskas CM, Smith RB, Meertens CM, Chang WL. 2007. Crustal deformation of the YellowstoneSnake River plain volcano-tectonic system; campaign and continuous GPS observations, 1987-2004. Journal of Geophysical Research 112(B03401)

Savage MK, Shih XR, Meyer RP, Aster RC, Buchbinder GGR, Ansorge J, Crampin S. 1989. Shear-wave anisotropy of active tectonic regions via automated $S$-wave polarization analysis. Tectonophysics 165(1-4):279-292.

Savage MK, Peppin W, Vetter U. 1990. Shear wave anisotropy and stress direction in and near Long Valley Caldera, California, 1979-1988. Journal of Geophysical Research 95(B7):11165-11177.

Savage MK. 1999. Seismic anisotropy and mantle deformation; what have we learned from shear wave splitting? Reviews of Geophysics 37(1):65-106.

Savage MK, Ohminato T, Aoki Y, Tsuji H, Greve SM. 2010. Stress magnitude and its temporal variation at Mt. Asama Volcano, Japan, from seismic anisotropy and GPS. Earth and Planetary Science Letters 290(3-4):403-414.

Shih X, Meyer R. 1990. Observation of shear wave splitting from natural events; south moat of Long Valley Caldera, California, June 29 to August 12, 1982. Journal of Geophysical Research 95(B7):11179-11195.

Silver PG, Chan WW. 1991. Shear wave splitting and subcontinental mantle deformation. Journal of Geophysical Research 96(B10):16429-16454. 
Silver PG, Long MD. 2011. The non-commutivity of shear wave splitting operators at low frequencies and implications for anisotropy tomography. Geophysical Journal International 184(3):1415-1427.

Smith RB, Arabasz WJ. 1991. Seismicity of the Intermountain Seismic Belt. Neotectonics of North America. Geological Society of America Decade Map (1), 185-228.

Smith RB, Jordan M, Steinberger B, Puskas CM, Farrell JM, Waite GP, Husen S, Chang W-L, O'Connell R. 2009. Geodynamics of the Yellowstone hot spot and mantle plume; seismic and GPS imaging, kinematics, and mantle flow. Journal of Volcanology and Geothermal Research 188(1-3):26-56.

Sneath PHA. 1957. The application of computers to taxonomy. Journal of General Microbiology 17:201-226.

Tai L-x, Gao Y, Cao F-j, Shi Y-t, Wu J, Jiao M-r. 2008. Shear-wave splitting beforeand after the 1999 Xiuyan earthquake in Liaoning, China. Acta Seismologica Sinica (English Edition) 21(4):344-357.

Teanby NA, Kenda NA, Kendall JM, van der Baan M. 2004. Automation of shear-wave splitting measurements using cluster analysis. Bulletin of the Seismological Society of America 94(2):453-463.

Titzschkau T, Savage M, Hurst T. 2010. Changes in attenuation related to eruptions of Mt. Ruapehu Volcano, New Zealand. Journal of Volcanology and Geothermal Research 190(1-2):168-178.

Vecsey L, Plomerova J, Babuska V. 2008. Shear wave splitting measurements; problems and solutions. Tectonophysics 462(1-4):178-196.

Waite GP, Smith RB. 2002. Seismic evidence for fluid migration accompanying subsidence of the Yellowstone Caldera. Journal of Geophysical Research 107(B9):2177.

Waite GP, Smith RB. 2004. Seismotectonics and stress field of the Yellowstone volcanic plateau from earthquake first-motions and other indicators. Journal of Geophysical Research 109(B02301).

Waite GP, Schutt DL, Smith RB. 2005. Models of lithosphere and asthenosphere anisotropic structure of the Yellowstone hot spot from shear wave splitting. Journal of Geophysical Research 110(B11304).

Waite GP, Chang WL. 2007. Shear-wave splitting from local earthquakes as an indicator of crustal stress at Yellowstone. Eos Trans. AGU 88(52):Fall Meet. Suppl., Abstact V53B1319.

White BJP, Smith RB, Husen S, Farrell JM, Wong I. 2009. Seismicity and earthquake hazard analysis of the Teton-Yellowstone region, Wyoming. Journal of Volcanology and Geothermal Research 188(1-3):277-296. 
Wicks CW, Thatcher W, Dzurisin D, Svarc J. 2006. Uplift, thermal unrest and magma intrusion at Yellowstone caldera. Nature 440(7080):72-75. 


\section{Appendix A: Cluster Analysis Details}

The technique for automated cluster analysis was adapted from Teanby et al. (2004) and is detailed below.

\subsection{Clustering}

Prior to clustering, the variables $\varphi$ and $\delta t$ need to be standardized so that equal weight is given to each variable when calculating the distance between clusters. If the variables are not standardized, variance in $\varphi$ will impact the clustering much more severely than variance in $\delta t$. We choose to standardize by variable range as this method has proven effective in many clustering applications (Milligan and Cooper 1988; Gnanadesikan et al. 1995). $\varphi$ is scaled to $180^{\circ}$ and $\delta t$ is scaled to $0.3 \mathrm{sec}$, the maximum allowable values used in the grid searches for splitting parameter calculations (Table 8.1). Herein, $\varphi$ and $\delta t$ will refer to the scaled measurements.

Teanby et al. (2004) employ an unsupervised single-linkage agglomerative hierarchical method (Sneath 1957) to cluster $N$ pairs of splitting parameters for each of the events . Following Teanby et al. (2004):

Consider the $N$ scaled measurements $\left(\delta t_{i}, \varphi_{i}\right)$ with variances $\left(\sigma_{\varphi_{i}}^{2} \sigma_{\delta t_{i}}^{2}\right), i=$ $1 \ldots N$. The data will be partitioned into $M$ clusters. In each cluster $C_{j}$, there are $N_{j}$ data points, where $j=1 \ldots M$... We start with the same number clusters as there are data points $(M=N)$ and calculate all of the intercluster distances. ... The intercluster distance is simply the rescaled [squared] Euclidean distance between cluster centers. ... The two nearest clusters are then combined so that the number of clusters decreases by one. We continue combining clusters until there is only one cluster $(M=$ 1) comprising the whole dataset. The result is a hierarchy of clusters. ...

For each number of clusters $M=1 \ldots N$, we calculate the number of datapoints $N_{j}$ in each cluster $C_{j}$ and the positions of the cluster centers $\left(\Delta t_{j}\right.$, $\left.\Phi_{j}\right)$, given by the mean position of points within the cluster: 


$$
\begin{gathered}
\Delta \mathbf{t}_{\mathrm{j}}=\frac{\sum_{\mathrm{i}=1}^{\mathrm{N}_{\mathrm{j}}} \delta \mathbf{t}_{\mathbf{i}}^{(\mathrm{j})}}{\mathbf{N}_{\mathrm{j}}}, \\
\Phi_{\mathrm{j}}=\frac{\sum_{\mathrm{i}=1}^{\mathbf{N}_{\mathrm{j}}} \varphi_{\mathrm{i}}^{(\mathrm{j})}}{\mathbf{N}_{\mathrm{j}}},
\end{gathered}
$$

where $\varphi_{i}^{(j)}$ and $\delta t_{i}^{(j)}$ are the measurements that belong to the cluster $j$.

\subsection{Selecting the Optimum Number of Clusters}

We wish to define the optimum number of clusters at some point well below the number of data points. Ideally, the data points will form few tight clusters. But, as our data is noisy and far from ideal, we need to define a maximum allowable number in order to prevent numerous spurious clusters. We set $M_{\max }=20$ which allows sufficient room for clustering of the scattered data without allowing too many clusters with small numbers of data points. Following Teanby et al. (2004):

To determine the [optimum] number of clusters, we use the methods of Caliński and Harabasz (1974) and Duda and Hart (1973). These were the top two performers in a comparison of 30 estimators of optimum cluster number by Milligan and Cooper (1985). Clustering is stopped when these criteria pass specific thresholds.

We define the within-cluster covariance $\mathbf{W}$ and the between-cluster covariance $\mathbf{B}$ as 


$$
\begin{aligned}
& \mathbf{W}=\left[\begin{array}{l}
\sum_{j=1}^{M} \sum_{i=1}^{N_{j}}\left(\delta t_{i}^{(j)}-\Delta t_{j}\right)^{2} \\
\sum_{j=1}^{M} \sum_{i=1}^{N_{j}}\left(\delta t_{i}^{(j)}-\Delta t_{j}\right)\left(\varphi_{i}^{(j)}-\Phi_{j}\right)
\end{array}\right. \\
& \sum_{\mathbf{j}=1}^{\mathbf{M}} \sum_{\mathbf{i}=1}^{\mathbf{N}_{\mathbf{j}}}\left(\delta \mathbf{t}_{\mathbf{i}}^{(\mathbf{j})}-\Delta \mathbf{t}_{\mathbf{j}}\right)\left(\varphi_{\mathbf{i}}^{(\mathbf{j})}-\Phi_{\mathbf{j}}\right) \\
& \sum_{\mathbf{j}=1}^{\mathbf{M}} \sum_{\mathbf{i}=1}^{\mathbf{N}_{\mathbf{j}}}\left(\varphi_{\mathbf{i}}^{(\mathbf{j})}-\Phi_{\mathbf{j}}\right)^{2} \\
& \mathbf{B}=\left[\begin{array}{ll}
\sum_{j=1}^{M}\left(\Delta t_{j}-\overline{\Delta t}\right)^{2} & \Phi \sum_{j=1}^{M}\left(\Phi \Delta t_{j}-\overline{\Delta t}\right)\left(j_{j}-\right) \\
\sum_{j=1}^{M}\left(\Delta t_{j}-\overline{\Delta t}\right)\left(\Phi_{j}-\bar{\Phi}\right) & \sum_{j=1}^{M}\left(\Phi_{j}-\bar{\Phi}\right)^{2}
\end{array}\right],
\end{aligned}
$$

where $\overline{\Delta t}$ and $\bar{\Phi}$ are the mean values of $\delta t$ and $\varphi$ over all the samples:

$$
\begin{aligned}
& \overline{-}=\frac{\sum_{\mathbf{i}=1}^{\mathbf{N}} \delta \mathbf{t}_{\mathbf{i}}}{\mathbf{N}}, \\
& \bar{\Phi}=\frac{\sum_{\mathbf{i}=1}^{\mathbf{N}} \varphi_{\mathbf{i}}}{\mathbf{N}} .
\end{aligned}
$$

The Caliński and Harabasz (1974) criterion is

$$
c(M)=\frac{(N-M) \operatorname{trace}(\mathrm{B})}{(M-1) \operatorname{trace}(\mathrm{W})}
$$

The maximum of this function indicates that the between-cluster variance is maximized with respect to the within-cluster variance, indicating tight clusters that are widely spaced. Therefore, the optimum number of clusters $M$ is obtained when $c(M)$ is maximized. 
The Duda and Hart (1973) criterion is based on the ratio of within-cluster variances when two clusters are combined into one cluster. The variance of the two individual clusters is given by

$$
\sigma_{2}^{2}=\sum_{\mathbf{j}=1}^{2} \sum_{\mathbf{i}=1}^{\mathbf{N}_{\mathrm{j}}}\left[\left(\delta \mathbf{t}_{\mathbf{i}}^{(\mathbf{j})}-\Delta \mathbf{t}_{\mathbf{j}}\right)^{2}+\left(\varphi_{\mathbf{i}}^{(\mathbf{j})}-\Phi_{\mathbf{j}}\right)^{2}\right],
$$

and the variance when the two clusters are combined into one cluster is

$$
\sigma_{1}^{2}=\sum_{\mathbf{i}=1}^{\mathbf{N}_{1}}\left[\left(\delta \mathbf{t}_{\mathbf{i}}^{(1)}-\Delta \mathbf{t}_{1}\right)^{2}+\left(\varphi_{\mathbf{i}}^{(1)}-\Phi_{1}\right)^{2}\right] .
$$

The null hypothesis is that the two clusters should be combined as a single cluster. Normally distributed within-cluster distances are assumed, and the null hypothesis is rejected when

$$
\left(1-\frac{\sigma_{2}^{2}}{\sigma_{1}^{2}}-\frac{2}{\pi \mathbf{p}}\right)\left(\frac{\mathbf{N}_{\mathrm{j}} \mathbf{p}}{2\left[1-\frac{8}{\pi^{2} \mathbf{p}}\right]}\right)^{\frac{1}{2}}>\mathbf{c}_{\text {critical }}
$$

Where $p$ is the number of parameters $(=2$, i.e., $\varphi$ and $\delta t)$ and $c_{\text {critical }}$ is the critical value from a standard normal distribution. Milligan and Cooper (1985) found that $c_{\text {critical }}=3.20$ gave the best results. We consider the hierarchy of cluster from $M=1 \ldots N$ and halt the subdivision of clusters when equation [(A10)] is no longer satisfied [or $M=M_{\max }$ ].

We used the maximum value of $M$ predicted by the two stopping criteria as the optimum number of clusters because in our case it is preferable to overestimate the number of clusters so that significantly different results are not included in the same cluster. 


\subsection{Selecting the Optimum Cluster and Measurement}

From the clusters determined by the methods above, we wish to select the optimum cluster and corresponding measurement. From Teanby et al. (2004):

Criteria for the best cluster are based on the number of points and the variance within the cluster. All clusters with fewer than data points are considered spurious and rejected. If this leaves no cluster, there is no stable solution.

In Teanby et al. (2004), $N_{c_{\text {min }}}=10$ was chosen such that the minimum number of points in each cluster was approximately the number of sample points in one cycle. In our case, $N_{c_{\text {min }}}=20-25$, approximately one cycle's worth of points, would result in too few stable results, thus $=10$ was chosen. From Teanby et al. (2004):

The within-cluster variance $\sigma_{c_{j}}^{2}$ and mean data variance, $\sigma_{d_{j}}^{2}$ of the remaining clusters are then calculated according to

$$
\begin{aligned}
& \sigma_{\mathbf{c}_{j}}^{2}=\frac{\sum_{\mathbf{i}=1}^{\mathbf{N}_{\mathrm{j}}}\left(\delta \mathbf{t}_{\mathbf{i}}^{(\mathrm{j})}+\Delta \mathbf{t}_{\mathbf{j}}\right)^{2}+\left(\varphi_{\mathrm{i}}^{(\mathrm{j})}+\Phi_{\mathrm{j}}\right)^{2}}{\mathbf{N}_{\mathbf{j}}}, \\
& \sigma_{\mathbf{d}_{\mathrm{j}}}^{2}=\left[\sum_{\mathrm{i}=1}^{\mathrm{N}_{\mathrm{j}}} \frac{1}{\left(\sigma_{\delta \mathrm{t}_{\mathrm{i}}}\right)^{(\mathrm{j})}}\right]^{-1}+\left[\sum_{\mathrm{i}=1}^{\mathrm{N}_{\mathrm{j}}} \frac{1}{\left(\sigma_{\varphi_{\mathrm{i}}}^{(\mathrm{j})}\right)^{2}}\right]^{-1} .
\end{aligned}
$$

Equation [(A1.12)] is related to the harmonic mean, which reduces the effect of outliers....We define an overall variance for the cluster $\sigma_{o_{j}}^{2}$, which is set to $\max \left(\sigma_{c_{j}}^{2}, \sigma_{d_{j}}^{2}\right)$. The best cluster has the smallest value of $\sigma_{o_{j}}^{2}$. Although $\sigma_{o_{j}}^{2}$ is a simple measure of cluster quality, minimizing $\sigma_{o_{j}}^{2}$ avoids the selection of diffuse clusters with low measurement errors and tight clusters with high measurement errors. ... The best measurement is simply the measurement with the smallest variance from within the best clusters. 
Table 8.1 Cluster Analysis Parameters

\begin{tabular}{|l|l|}
\hline $\begin{array}{l}\text { Cluster Analysis } \\
\text { Parameters }\end{array}$ & Value \\
\hline$\varphi$ scale & $180^{\circ}$ \\
\hline$\delta t$ scale & $0.3 \mathrm{sec}$ \\
\hline$M_{\max }$ & 20 \\
\hline$C_{\text {critical }}$ & 3.20 \\
\hline & 10 \\
\hline
\end{tabular}




\title{
9. Appendix B: Figure 1.2 Copyright License Agreement
}

\author{
THE AMERICAN ASSOCIATION FOR THE ADVANCEMENT OF SCIENCE \\ LICENSE \\ TERMS AND CONDITIONS
}

Apr 29, 2011

This is a License Agreement between Nicole D McMahon ("You") and The American Association for the Advancement of Science ("The American Association for the Advancement of Science") provided by Copyright Clearance Center ("CCC"). The license consists of your order details, the terms and conditions provided by The American Association for the Advancement of Science, and the payment terms and conditions.

\section{All payments must be made in full to CCC. For payment instructions, please see information listed at the bottom of this form.}

License Number

License date
2658290363383

Apr 29, 2011
Licensed content

publication

Licensed content title

Licensed content author

Licensed content date

Volume number

Issue number

Type of Use

Requestor type

Format

Portion

Number of figures/tables

Order reference number
Science

Accelerated Uplift and Magmatic Intrusion of the Yellowstone Caldera, 2004 to 2006

Wu-Lung Chang, Robert B. Smith, Charles Wicks, Jamie M. Farrell, Christine M. Puskas

Nov 9, 2007

318

5852

Thesis / Dissertation

Other Individual

Print and electronic

Figure

1 
Title of your thesis / dissertation

Expected completion date May 2011

Estimated size(pages) 71

Total 0.00 USD 